\title{
HIGH-EFFICIENCY SWITCHED-MODE POWER AMPLIFIER USING GALLIUM NITRIDE ON SILICON HEMT TECHNOLOGY
}

\author{
By \\ Harpreet Panesar
}

\begin{abstract}
A thesis
presented to Carleton University

in partial fulfilment of the

thesis requirement for the degree of

MASTER OF APPLIED SCIENCE

in
\end{abstract}

ELECTRICAL ENGINEERING

Ottawa, Ontario, Canada

(C) Harpreet S. Panesar, 2007 


$\begin{array}{ll}\begin{array}{l}\text { Library and } \\ \text { Archives Canada }\end{array} & \begin{array}{l}\text { Bibliothèque et } \\ \text { Archives Canada }\end{array} \\ \begin{array}{l}\text { Published Heritage } \\ \text { Branch }\end{array} & \begin{array}{l}\text { Direction du } \\ \text { Patrimoine de l'édition }\end{array} \\ \begin{array}{l}\text { 395 Wellington Street } \\ \text { Ottawa ON K1A ON4 } \\ \text { Canada }\end{array} & \begin{array}{l}\text { 395, rue Wellington } \\ \text { Ottawa ON K1A ON4 } \\ \text { Canada }\end{array}\end{array}$

Your file Votre référence ISBN: 978-0-494-33664-9 Our file Notre référence ISBN: 978-0-494-33664-9

NOTICE:

The author has granted a nonexclusive license allowing Library and Archives Canada to reproduce, publish, archive, preserve, conserve, communicate to the public by telecommunication or on the Internet, loan, distribute and sell theses worldwide, for commercial or noncommercial purposes, in microform, paper, electronic and/or any other formats.

The author retains copyright ownership and moral rights in this thesis. Neither the thesis nor substantial extracts from it may be printed or otherwise reproduced without the author's permission.
AVIS:

L'auteur a accordé une licence non exclusive permettant à la Bibliothèque et Archives Canada de reproduire, publier, archiver, sauvegarder, conserver, transmettre au public par télécommunication ou par l'Internet, prêter, distribuer et vendre des thèses partout dans le monde, à des fins commerciales ou autres, sur support microforme, papier, électronique et/ou autres formats.

L'auteur conserve la propriété du droit d'auteur et des droits moraux qui protège cette thèse. $\mathrm{Ni}$ la thèse ni des extraits substantiels de celle-ci ne doivent être imprimés ou autrement reproduits sans son autorisation.
In compliance with the Canadian

Privacy Act some supporting forms may have been removed from this thesis.

While these forms may be included in the document page count, their removal does not represent any loss of content from the thesis.
Conformément à la loi canadienne sur la protection de la vie privée, quelques formulaires secondaires ont été enlevés de cette thèse.

Bien que ces formulaires aient inclus dans la pagination, il n'y aura aucun contenu manquant. 


\section{Abstract}

The continuing trend toward greater capacity and higher data rates in wireless communication systems places increasing demands on the radio frequency $(\mathrm{RF})$ power provided by base station transmitters. Conventional RF power amplifiers (PAs) in use today have poor operating efficiencies and require considerable additional power and volume for heat removal. Research on more efficient PA technology is therefore important to the growth of the wireless industry.

This thesis investigates high-efficiency switched-mode microwave power amplification using a new transistor technology, the Gallium Nitride on Silicon High Electron Mobility Transistor (GaN-on-Si HEMT), which promises to deliver high output power levels at lower cost than the alternative GaN-on-SiC technology. The principles of power devices and power amplification modes are discussed. A new large-signal equivalent model is developed for a $2 \mathrm{~mm}$ GaN-on-Si HEMT over the $0 \mathrm{GHz}-20 \mathrm{GHz}$ range, based on measured data. The chosen EEsof GaAs nonlinear HEMT model reproduces the GaN transistor's behavior reasonably well over a large range of bias conditions, signal levels, and frequencies, although several parameter assumptions are made. Based on this model, the design, optimization, fabrication and testing of a $3.5 \mathrm{GHz}, 1 \mathrm{~W}$, Class E power 
amplifier proceeds. The PA design is successful, with measurements revealing a gain of $10.7 \mathrm{~dB}$, with an output power of $942 \mathrm{~mW}$, and a power-added efficiency of $40.4 \%$.

This work shows the suitability of lower cost GaN-on-Si transistor technology for switched-mode microwave power amplifiers with possible applications in WiMAX base stations. It is recommended that further device modeling and other switched-mode amplifier topologies be investigated. 


\section{Acknowledgements}

Although this thesis represents my own contribution to science, this work would not have been possible without the guidance and support of many people.

I would like to express my deepest gratitude to my thesis supervisors, Professor Langis Roy and Professor Tom Smy, for their support and patience throughout this project. I would also like to thank Steven McGarry; his expertise and seemingly boundless technical knowledge have contributed a great deal to the success of this work.

I am grateful to the many organizations, including Nitronex Corporation (Durham, N.C.), the Department of Mechanical and Aerospace Engineering at Carleton University (Ottawa, ON), the Communications Research Centre (Ottawa, ON), and Nortel (Ottawa, $\mathrm{ON})$, for their contributions in all aspects of this project.

It has been a privilege sharing this experience with my friends and fellow graduate students, Charles Berndt, Steven Penney, Céline Fletcher, Daniel Olsiewski, Peter Chyurlia, Fiona Shearer, and Justin Abbott. I thank Janice Yu and Mike Sowka for the many fruitful discussions in the final months of manuscript preparation.

I owe thanks to my family for their unwavering support and understanding over the course of my academic career. 


\section{Table of Contents}

$\begin{array}{ll}\text { Abstract } & \text { ii }\end{array}$

Acknowledgements $\quad$ iv

Table of Contents $\quad$ v

List of Tables $\quad$ vii

List of Figures $\quad$ viii

1 Introduction $\quad 1$

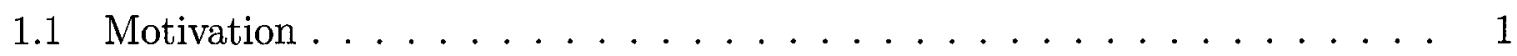

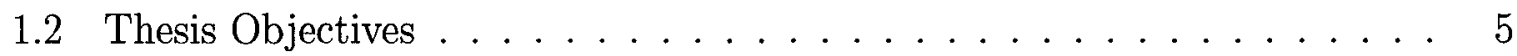

1.3 Research Partnership . . . . . . . . . . . . . . . . 5

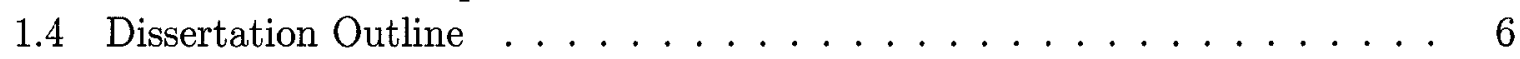

2 Technology Review $\quad 8$

2.1 Conventional MOSFETs and HEMTs . . . . . . . . . . . . . 8

2.2 Comparison with Wide Bandgap HEMTs . . . . . . . . . . . . 10

3 Microwave Power Amplifiers $\quad 14$

3.1 On Power, Bandwidth, and Linearity . . . . . . . . . . . . . . . . 14

3.2 Conventional Power Amplifiers . . . . . . . . . . . . . . . 15

3.3 Class E Switched-Mode Power Amplifier . . . . . . . . . . . . . . . . . . . 22

3.3 .1 Idealized Analysis . . . . . . . . . . . . . . . 23

3.3.2 The Class E Concept at $\mathrm{GHz}$ Frequencies . . . . . . . . . . . 29

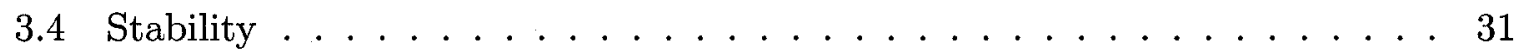

3.5 Previous Work and Proposed Amplifier Specifications . . . . . . . . . . 32

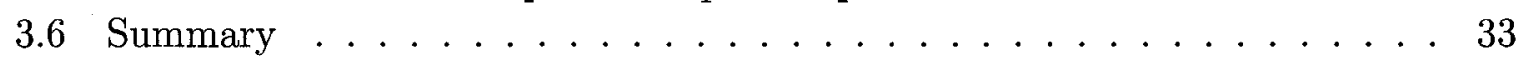

4 Gallium Nitride High Electron Mobility Transistor Nonlinear Model 36

4.1 The Nitronex GaN-on-Si HEMT . . . . . . . . . . . . . . . . . 38

4.2 EEsof $2 \mathrm{~mm}$ Nonlinear HEMT Model . . . . . . . . . . . . . 39 
4.2.1 Drain-Source Current Model . . . . . . . . . . . . . . . . . . 41

4.2 .2 Dispersion Model . . . . . . . . . . . . . . . . . . . 41

Charge Trapping . . . . . . . . . . . . . . . 41

Self-Heating . . . . . . . . . . . . . . . 42

4.2 .3 Gate and Output Charge Models . . . . . . . . . . . . 43

4.2.4 Gate Forward Conduction and Breakdown Model . . . . . . . . . 43

4.3 Transistor Characterization . . . . . . . . . . . . . . . . . 44

4.3 .1 Extrinsic Parameters . . . . . . . . . . . . . . . . . 45

4.3.2 Drain-Source Current and Transconductance . . . . . . . . . . 49

4.3.3 Gate Forward Conduction . . . . . . . . . . . . . . 53

4.3 .4 Gate and Output Capacitance . . . . . . . . . . . . 54

4.4 Model Verification . . . . . . . . . . . . . . . . . . . . 60

4.5 Summary . . . . . . . . . . . . . . . . 66 66

5 Class E GaN-on-Si Microwave Power Amplifier 68

5.1 Ideal Class E Power Amplifier . . . . . . . . . . . . . . . . . . 70

5.2 The Class E Load Network . . . . . . . . . . . . . . . . . . . . . 73

5.2 .1 Lumped Element Analysis . . . . . . . . . . . . . . . . 74

5.2 .2 Microstrip Transmission Line Implementation . . . . . . . . . . . 81

5.3 Input Matching Network . . . . . . . . . . . . . . . . . 82

5.4 Bias Insertion Network and Stability . . . . . . . . . . . . . . . 89

5.4 .1 Power Supply Decoupling . . . . . . . . . . . . . . . . . 93

5.4.2 RF Loading and Oscillation Suppression . . . . . . . . . . . . 94

5.4 .3 Protection and Stability . . . . . . . . . . . . . . . . 96

5.4.4 Complete Circuit Simulation . . . . . . . . . . . . . . . . . . 97

5.5 Thermal Considerations . . . . . . . . . . . . . . . . . . 99

5.6 Simulated and Measured Results . . . . . . . . . . . . . . . . . 99

5.7 Summary . . . . . . . . . . . . . . . . . . . 106

6 Conclusions and Future Work 108

Appendix A Agilent EEHEMT 2 mm GaN-on-Si Nonlinear Model 111

References 112 


\section{List of Tables}

$2.1 \mathrm{Si}, \mathrm{GaAs}, \mathrm{SiC}$, and $\mathrm{GaN}$ Material Properties . . . . . . . . . . . 11

2.2 Best Reported High-Speed AlGaAs/GaAs, InP, and AlGaN/GaN HEMTs . 11

2.3 Best Reported Power Capabilities for AlGaAs/GaAs, InP, and AlGaN/GaN HEMTs . . . . . . . . . . . . . . . . . . . 12

3.1 Conduction Angles and Maximum Theoretical Drain Efficiencies for Conventional PAs . . . . . . . . . . . . . . . . . 21

$4.12 \mathrm{~mm}$ GaN-on-Si HEMT Specifications . . . . . . . . . . . . . 38

4.2 Optimized Extrinsic Element Values . . . . . . . . . . . . . . . . 49

4.3 Summary of Optimized Model Parameters from $I-V$ Analysis . . . . . . . 51

4.4 Summary of Optimized Model Parameters from DC $g_{m}$ Analysis . . . . . . 53

4.5 Summary of Optimized Parameters for the Schottky Diode Model . . . . . 54

4.6 Summary of Optimized Parameters for the Charge Models . . . . . . . . . 60

5.1 PA Specifications and Substrate Material Properties . . . . . . . . . . 69

5.2 Class E PA Performance for Small- and Large-Signal Input Matching Circuits 88

5.3 Simulated Versus Measured Power Amplifier Performance . . . . . . . . . . 105

5.4 Comparison of this Work with Other GaN PAs . . . . . . . . . . . 106 


\section{List of Figures}

2.1 AlGaN/GaN HEMT Physical Structure . . . . . . . . . . . . . . 10

3.1 Conventional PA Load-Lines and Drain Current Profiles . . . . . . . 16

3.2 Generic Power Amplifier Model . . . . . . . . . . . . . . . . . . . . 17

3.3 Generic Power Amplifier Voltage and Current Waveforms . . . . . . . . 18

3.4 Ideal Class E PA Model . . . . . . . . . . . . . . . . . . . . . . 24

3.5 Ideal Class E PA Voltage and Current waveforms . . . . . . . . . . 25

4.1 Top-Level Layout of the Nitronex $2 \mathrm{~mm}$ GaN-on-Si HEMT . . . . . . . . 38

4.2 Agilent EEsof HEMT Nonlinear Model Schematic . . . . . . . . . . . . . . 40

4.3 HEMT Small-Signal Model . . . . . . . . . . . . . . . . . . . . 45

4.4 Gate Region of a HEMT When $V_{d s}=0 \mathrm{~V} \ldots \ldots \ldots \ldots . \ldots 45$

4.5 Measured $\Re\left\{Z_{11}\right\}$ vs. $\frac{1}{I_{g}}$ at $1 \mathrm{GHz}$ for $V_{d s}=0 \mathrm{~V} \ldots \ldots \ldots \ldots$

4.6 Symbolic Form of Extrinsic Z-Parameters . . . . . . . . . . . . . 47

4.7 Imaginary Parts of $Z_{11}, Z_{12}$, and $Z_{22}$ vs. Frequency . . . . . . . 48

4.8 Extrinsic Parameters Extracted from Small-Signal Model Optimization . . 49

4.9 Pulsed Current-Voltage Characteristics . . . . . . . . . . . . . 50

4.10 Pulsed DC $g_{m}$ Measurement at $V_{d s}=20 \mathrm{~V} \ldots \ldots \ldots \ldots \ldots$

4.11 Forward Gate-Source Current Measurement . . . . . . . . . . . . . . 54

4.12 Input Capacitance $\left(C_{11}\right)$ vs. $V_{g s} \ldots \ldots \ldots \ldots \ldots \ldots$

4.13 Input Capacitance $\left(C_{11}\right)$ vs. $V_{d s} \ldots \ldots \ldots \ldots \ldots \ldots \ldots$

4.14 Gate-Drain Capacitance in Saturation $\left(C_{g d s a t}\right)$ vs. $V_{g s} \ldots \ldots \ldots . \ldots 58$

4.15 Output Capacitance $\left(C_{d s o}\right)$ vs. $V_{d s} \ldots \ldots \ldots \ldots \ldots \ldots \ldots$

4.16 Modeled vs. Measured comparison of $I_{d s}$ vs. $V_{d s} \ldots \ldots \ldots \ldots \ldots$

4.17 Measured and Simulated DC $g_{m}$ and $I_{d s}$ w.r.t. $V_{g s} \ldots \ldots \ldots 6$

4.18 Simulated/Measured S-Parameter Response for $V_{d s}=20 \mathrm{~V}$ and $V_{g s}=-1 \mathrm{~V} 64$

4.19 Simulated/Measured Model Deviations . . . . . . . . . . . . . . 64

4.20 Simulated/Measured GaN Schottky Diode Characteristic . . . . . . . . . 65

4.21 3.5 GHz Class A Measured Load Pull Contours of the $2 \mathrm{~mm}$ GaN HEMT . 66

5.1 System Level Diagram of a Class E PA Module . . . . . . . . . . . . . 69

5.2 Original Class E PA Schematic . . . . . . . . . . . . . . 70

5.3 Simulated Voltages and Currents of an Ideal Class E Power Amplifier . . . 72

viii 
5.4 Class E Load Network and Output Bias Insertion Unit . . . . . . . . . 74

5.5 Load Impedance as Seen by the HEMT . . . . . . . . . . . . . . 75

5.6 Lumped Element Class E GaN PA with Square-Wave Input Drive . . . . . 76

5.7 Class E Drain Voltage Superimposed by Ideal Square-Wave Gate Driving Voltage . . . . . . . . . . . . . . . . . . 78

5.8 Simulated $I_{D S}, V_{D S}$ Waveforms of the Class E GaN PA . . . . . . . . . 79

5.9 Lumped Element Class E PA with Adjusted Load Network . . . . . . . . . 80

5.10 Double-Stage Class E Load Network . . . . . . . . . . . . . . 82

5.11 Microstrip Transmission Line Class E Load Network . . . . . . . . . . . . . 83

5.12 Simulated $S_{11}$ of the Microstrip Class E Load Network . . . . . . . . . . 84

5.13 Lumped Element Class E PA with Conjugate Input Matching Circuit . . . 85

5.14 Simulated $S_{11}$ of the Class E PA with Conjugate Input Matching Circuit . 86

5.15 Lumped Element Class E PA with Power Matched Input . . . . . . . . . . 87

5.16 Simulated Large-Signal $S_{11}$ of the Class E PA with Power Matched Input . 88

5.17 Input Matching Circuit Layout . . . . . . . . . . . . . . . . . . 90

5.18 System-Level Schematic of the Bias Insertion Circuits . . . . . . . . . . . 91

5.19 Block Diagram of Bias Network . . . . . . . . . . . . . . . . 92

5.20 Power Supply Decoupling Circuit . . . . . . . . . . . . . . . . . 94

$5.21 S_{11}$ Response of the Power Supply Decoupling Circuit . . . . . . . . . . . 94

5.22 An RF Choke and Noise Suppression Circuit . . . . . . . . . . . . . . 95

5.23 Simulations of RF Choke and Ferrites . . . . . . . . . . . . . . . . 96

5.24 Simulations of the Complete Bias Insertion Circuit . . . . . . . . . . 97

5.25 Small-Signal Stability Simulation . . . . . . . . . . . . . . . . . . . . . . . . . . . . . .

5.26 Aluminum Base Plate Design . . . . . . . . . . . . . . . . . 99

5.27 Fully Assembled $3.5 \mathrm{GHz}$ GaN-on-Si Class E Power Amplifier . . . . . . . 100

5.28 Small- and Large-Signal Test Sets . . . . . . . . . . . . . . . . . . . . . . . . . . . . . . . . . . .

5.29 Measured and Simulated $S_{11}$ Responses . . . . . . . . . . . . . 101

5.30 Measured $G_{T}, P A E$ vs. Frequency . . . . . . . . . . . . . . 102

5.32 Measured vs. Simulated Comparison of Class E PA Performance . . . . . . 104

A.1 $2 \mathrm{~mm}$ GaN-on-Si HEMT Nonlinear Model Component . . . . . . . . . . 111 


\section{Chapter 1}

\section{Introduction}

In efforts to reduce microwave communication transmitter cost while meeting stringent efficiency and power requirements, microwave designers are looking towards gallium nitride $(\mathrm{GaN})$ as a potential replacement for today's heavily taxed silicon (Si)-based microwave base station systems. This work addresses the pressing need for power amplifiers (PAs) to cost-effectively support emerging broadband, high frequency applications.

This chapter presents the motivation for pursuing high efficiency power amplification along with thesis objectives and an outline of the remaining chapters. The work in this thesis was made possible through a research partnership with Nitronex Corporation, Durham N.C. A short introduction into the nature of this partnership is also given.

\subsection{Motivation}

As wireless technology advances and society becomes increasingly familiarized with the myriad of potential wireless services, microwave systems designers are faced with the challenging task of building the foundation to support continually increasing commercial, 
military, and consumer demands.

Broadband (6 Mbps and greater) internet access, for example, is primarily available through Transmission Level 1 (T1), Digital Subscriber Line (DSL), or cable-modem connections [1]. The availability of telecommunication services in any country depends on many economic factors, such as population density, availability of service providers, and potential for profit. While broadband services may be available in large metropolitan centres and small cities, rural areas and developing countries lack the wireline backbone that is commonplace in many areas. The cost of building optical fiber- or cable-based infrastructure is impossibly high in places where it is non-existent, and service providers do not see significant return-on-investment for wireline implementation, leaving many communities without broadband access [2].

The WiMAX (Worldwide Interoperability for Microwave Access) broadband initiative is based on the IEEE 802.16 family of standards. Established in 2001, the WiMAX Forum is the driving force behind its vision to provide broadband multimedia access to any wireless terminal, anywhere, at any time. With a range of up to fifty kilometers, WiMAX is a point-to-multipoint standardized wireless networking solution supporting licensed and unlicensed frequency bands from 2 to $11 \mathrm{GHz}$ for non-line-of-sight (NLOS) operation, and from 10 to $66 \mathrm{GHz}$ for line-of-sight (LOS) transmission [3]. Once fully deployed, WiMAX will serve a variety of applications, including that of providing relatively affordable broadband coverage to underserved communities and developing countries.

Technological advances, such as those ushered in by WiMAX technology, promise to offer more and more broadband services to the end-user. This increased functionality has 
thus far been made possible by stretching the limits of existing solid-state power amplifiers in applications such as cellular base station and satellite transponders, or by using bulky traveling wave tube amplifiers in radar and navigation systems $[4,5]$. All of this has been done without significant reduction in component size (in the case of the vacuum tube) or improvements in transmitter efficiency (for solid-state implementations) and complexity, making the power amplifier the most expensive component in any radio front-end $[6,7]$.

Power amplifiers are used in a wide variety of applications from smart weapons systems in the military sector to wireless local area networks (WLANs) and automotive radar in the commercial and consumer markets. In their current state, however, many high frequency power amplifiers lose up to ninety percent of their applied DC power as heat, leading to low DC-RF conversion efficiency [5]. This problem of converting DC power to useful RF energy is further exacerbated by emerging broadband air interface standards that require low signal distortion at high power levels. Consequently, designers are forced to build inefficient power combined amplifiers or implement additional digital circuitry in order to minimize adjacent-channel spillover to conserve valuable spectrum space.

At the heart of many of these power amplifiers is the laterally-diffused metal-oxide semiconductor field-effect transistor (LDMOSFET). While the LDMOSFET PA does well with voice and data transmission, it requires complex linearization schemes in order to meet linearity, efficiency, and output power specifications [7,8]. The continued migration towards high frequency spectrum space has recently put the LDMOSFET's capabilities into question. Although high frequency operation can, indeed, be attained with gallium arsenide (GaAs), indium phosphide ( $\mathrm{InP}$ ), or silicon germanium ( $\mathrm{SiGe}$ ) amplifiers, their 
usefulness is limited to primarily the low power, low voltage regimes [5]. These solutions are costly and can only temporarily address a limited range of applications before the entire RF infrastructure must be revamped at the transistor level. In this regard, GaN has recently seen international attention as a front runner in many electronic systems [9-11].

GaN belongs to the family of wide bandgap semiconductors, which includes silicon carbide ( $\mathrm{SiC})$ and diamond. The nature of these materials gives them the ability to support high supply voltages, translating into highly efficient (DC-RF conversion efficiencies greater than 50\%), high power (greater than $1 \mathrm{~W}$ ) generation. Since bulk GaN substrates are not available, GaN could historically only be grown on either SiC or sapphire substrates. Although sapphire is a low-cost solution, its high thermal impedance makes it less attractive than $\mathrm{SiC}$ for power applications. Of the wide bandgap semiconductors, only $\mathrm{SiC}$ and diamond are available in bulk format; they exhibit superior properties for high-frequency (greater than $1 \mathrm{GHz}$ ), high-power operation, but are difficult and expensive to produce, and yield small substrates that are full of intrinsic defects. Silicon is still the champion when it comes to highly refined substrates; it is more thermally conductive than GaAs and its relative maturity allows it to be produced in large diameters and high quantities. Fortunately, recent advances in semiconductor processing technology have allowed the growth of GaN high electron-mobility transistors (HEMTs) on low cost Si substrates [12].

If the ultimate goal is to continue to provide reliable wireless services to the end user at a reasonable cost, then the benefits of low-cost GaN-on-Si devices must be exploited. It is the quest for high-efficiency power amplification that has provided the inspiration to 
study switched-mode Class E GaN-based microwave power amplifiers in this work.

\subsection{Thesis Objectives}

This thesis revisits the decades-old high-efficiency switched-mode Class E PA [13]. With some modifications, the Class E approach is applied for the first time to a GaN-on-Si power amplifier circuit for operation in the WiMAX (IEEE 802.16-2004) band at 3.5 GHz. Linearity and wide bandwidth are critical design issues in many applications but, as applied to the Class E topology, are outside the scope of this work as per the discussion in Chapter 3. More appropriately then, this thesis endeavors to demonstrate a high-efficiency narrow-band GaN-on-Si Class E power amplifier. In support of this objective, a measurement-based nonlinear model is developed and verified, along with an examination of both conventional and switched-mode power amplifier topologies. A technology comparison between GaN and competing technologies is given in Chapter 2 .

\subsection{Research Partnership}

Nitronex Corporation, based out of Durham, North Carolina, is a world leader in the wireless communications industry. A spin-off from the College of Engineering at North Carolina State University in the mid-1990s, today Nitronex has become a pioneer in developing low-cost, high-performance, GaN-on-Si power transistors for emerging telecommunications markets.

In 2004, Nitronex and the Department of Electronics at Carleton University engaged in a collaborative research program to investigate the performance of GaN-on-Si HEMTs 
in Class E switched-mode power amplifiers. Nitronex generously supplied several test structures to make this project a success.

\subsection{Dissertation Outline}

The remainder of this thesis expands upon the motivation and objectives discussed in the preceding sections, ultimately leading to a full hardware implementation of a Class E power amplifier. But to appreciate the hardware, one must fully understand the device technology, the theory that governs its operation, and the necessary design steps taken to reach the end goal.

Chapter 2 shows how GaN-based power transistors compare against other, more established device technologies. A comparison is done between material properties, cut-off frequencies, and power capability.

Chapter 3 presents standard microwave power amplifier theory with special attention given to the operation of the Class E power amplifier. Practical limitations of switchedmode power amplifiers operating at $\mathrm{GHz}$ frequencies are discussed. An explanation of stability, both in the small-signal regime and in the context of nonlinear power amplifiers, is also given. The chapter concludes with a summary of recent work in Class E power amplifiers along with proposed specifications for the design in Chapter 5

Chapter 4 delves into the nonlinear model of the GaN HEMT on which the PA in Chapter 5 is based. All aspects of the measurement-based device model are covered first, followed by complete small- and large-signal transistor characterization of the GaN device. A simulated versus measured comparison is done to validate the model. 
Chapter 5 presents a fully functional, $3.5 \mathrm{GHz}$ high-efficiency Class E power amplifier. Starting with idealized lumped-element simulations, the design moves forward through practical aspects, and ends with a physically realizable microstrip implementation. One of the key aspects to the design is ensuring that the method of bias insertion does not compromise performance. A detailed design of input and output bias circuits, complete with oscillation suppression, power supply decoupling, and failure protection circuitry, is presented. And finally, when dealing with appreciable power levels, thermal effects are inevitable, so a heat-sink design is also shown to directly remove heat from the GaN device. The complete design is fabricated, assembled, and demonstrated at the end of the chapter.

The conclusions of this thesis are given in Chapter 6. Given the proliferation of wide bandgap semiconductors such as GaN, recommendations for continuing research into switched-mode PA topologies are also provided. 


\section{Chapter 2}

\section{Technology Review}

Microwave transistor technology has evolved over the years from the basic metal-semiconductor field-effect transistor (MESFET) in the 1950s to current high-performance III-V (GaAs and $\mathrm{InP}$ ) and wide bandgap (AlN, $\mathrm{SiC}$, and $\mathrm{AlGaN}$ ) HEMTs. This chapter explores device technology for its usefulness in high frequency, power systems.

\subsection{Conventional MOSFETs and HEMTs}

III-V HEMTs offer high frequency performance, but suffer from low power densities in comparison to emerging wide bandgap technology. The Si LDMOSFET supports higher breakdown voltages and is an established technology used in most modern base station microwave power amplifiers. Beyond about $3 \mathrm{GHz}$ however, high parasitic capacitance puts the usefulness of LDMOSFETs in base station systems into question.

Until about 1980, typical HEMTs consisted of the AlGaAs/GaAs heterostructure. The two-dimensional electron gas (2DEG), which is the defining feature of HEMT structures, offers high carrier mobilities, but that alone is not enough to qualify a device with superior 
microwave performance. The AlGaAs/GaAs heterostructure does not have a large enough conduction band offset to allow good electron confinement with high 2DEG sheet charge density $\left(n_{s}\right)$. These are the qualities necessary for high-frequency operation.

The deficiencies of the AlGaAs/GaAs heterojunction led to advancements in material systems with larger conduction band offsets, such as lattice-matched heterostructures on InP with InGaAs channel layers, to facilitate higher frequency operation. Heterostructures incorporating InGaAs channel layers have conduction band offsets as high as $0.66 \mathrm{eV}$ compared to only $0.22 \mathrm{eV}$ for GaAs. The result is that $\mathrm{InP}$ devices can support $n_{s}$ and low-field carrier mobilities $\left(\mu_{0}\right)$ two to three times higher than conventional GaAs HEMTs, allowing them to attain record cut-off frequencies $\left(f_{T} s\right)$ and maximum frequencies of oscillation $\left(f_{\max } \mathrm{s}\right)$ of $340 \mathrm{GHz}$ and $600 \mathrm{GHz}$, respectively [14].

In addition to frequency performance of microwave devices, power capability is also an important figure of merit. Reports have shown a $2 \mathrm{GHz}, 240 \mathrm{~W}$, four-stage power amplifier using AlGaAs/GaAs HEMTs [15]. Power amplifiers using InP devices show reduced output power levels by a factor of least two at comparable frequencies. Si LDMOSFETs currently hold the largest marketshare in commercial base station power amplifiers. They are a low-cost technology offering high output power and breakdown voltage up $3 \mathrm{GHz}$. Typical drain-source breakdown voltages are in the $20 \mathrm{~V}$ to $40 \mathrm{~V}$ range, with $f_{T}$ and $f_{\max }$ anywhere between $5 \mathrm{GHz}$ and $15 \mathrm{GHz}$. Single Si LDMOSFETs with up to $220 \mathrm{~W}$ of output power at $2 \mathrm{GHz}$ have been reported in the literature [8]. There is a clear trade-off between speed and breakdown voltage: as gate length becomes smaller to achieve higher $f_{T}$, the physical space between the gate and drain becomes shorter, requiring the device 
to support a larger gate-drain electric field.

\subsection{Comparison with Wide Bandgap HEMTs}

Wide bandgap transistors, AlGaN/GaN HEMTs in particular, are an emerging technology that promise to deliver both high frequency and high power operation at larger power densities than existing III-V semiconductors. Figure 2.1 shows a cross-sectional view of the typical AlGaN/GaN HEMT physical structure. The Al content in the AlGaN causes

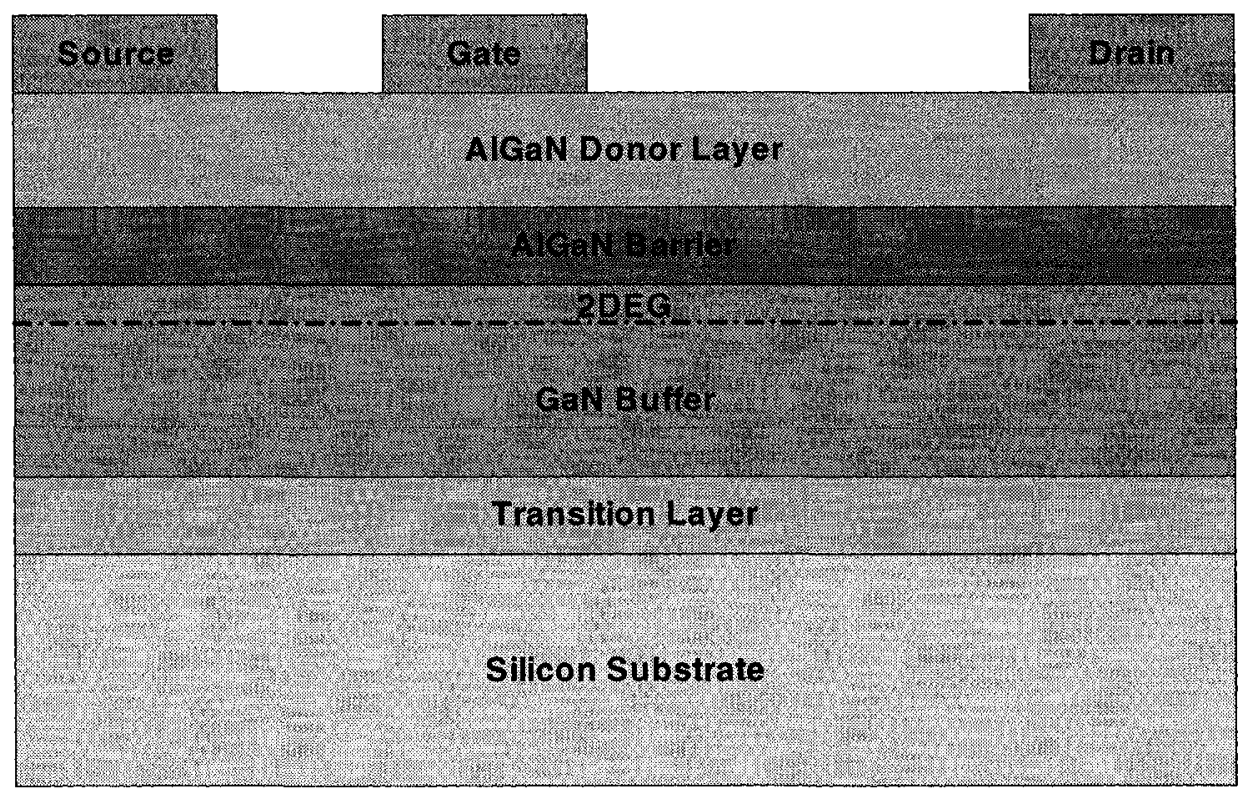

Figure 2.1: Physical structure of an AlGaN/GaN HEMT.

a high-purity 2DEG to form at the heterointerface towards the GaN layer. In comparison to AlGaAs/GaAs systems with 2DEG electron densities at around $2(10)^{12} \mathrm{~cm}^{-2}$, the sheet charge density in the AlGaN/GaN 2DEG is about five times higher at $10^{13} \mathrm{~cm}^{-2}$. This property, combined with high electron mobility, high electron saturation velocity, and the material's ability to support high breakdown electric field, allows AlGaN/GaN 
HEMTs to produce high RF power levels at high frequencies. Table 2.1 summarizes some of the key material properties of $\mathrm{GaN}$ compared to $\mathrm{Si}, \mathrm{GaAs}$, and the most common $\mathrm{SiC}$ crystal structure. The high thermal conductivity of wide bandgap materials makes them

\begin{tabular}{|c|c|c|c|c|}
\hline Parameter & $\mathrm{Si}$ & $\mathrm{GaAs}$ & $\mathrm{SiC}$ & $\mathrm{GaN}$ \\
\hline \hline Energy gap $[\mathrm{eV}]$ & 1.11 & 1.43 & 3.2 & 3.4 \\
\hline Breakdown field $[\mathrm{V} / \mathrm{cm}]$ & $5.7(10)^{5}$ & $6.4(10)^{5}$ & $3.3(10)^{6}$ & $3.8(10)^{6}$ \\
\hline Electron mobility $\left[\mathrm{cm}^{2} / \mathrm{Vs}\right]$ & 1350 & 6000 & 800 & 1600 \\
\hline Electron peak velocity $\left[10^{7} \mathrm{~cm} / \mathrm{s}\right]$ & 1 & 1.8 & 2 & 2.5 \\
\hline Thermal conductivity $[\mathrm{W} / \mathrm{Kcm}]$ & 1.5 & 0.46 & 3.5 & 1.7 \\
\hline
\end{tabular}

Table 2.1: Material properties of $\mathrm{Si}, \mathrm{GaAs}, \mathrm{SiC}$, and $\mathrm{GaN}$

especially attractive for base station applications; their reliability at higher temperatures could reduce much of costs associated with cooling equipment.

Within the last ten years, the scientific community has seen advancements on the theory and techniques of reliably processing $\mathrm{GaN}$ on various substrates, including $\mathrm{SiC}$, sapphire, and $\mathrm{Si}$. At present, GaN grown on $\mathrm{SiC}$ is the single commonality among most GaN-based microwave circuits. $\mathrm{SiC}$ is more thermally conductive than $\mathrm{Si}$ and GaAs. Moreover, the energy gap at the heterointerface provides superior 2DEG electron confinement. As a result, AlGaN/GaN-on-SiC HEMTs have shown excellent high frequency performance and record power densities. Table 2.2 gives a comparison of some of the best cut-off frequencies reported for AlGaN/GaN, AlGaAs/GaAs, and InP HEMT devices. A

\begin{tabular}{|c|c|c|c|c|}
\hline Transistor & $L_{g}[\mu \mathrm{m}]$ & $f_{T}[\mathrm{GHz}]$ & $f_{\max }[\mathrm{GHz}]$ & Ref. \\
\hline \hline AlGaAs/GaAs HEMT & 0.1 & 113 & 110 & {$[16]$} \\
\hline InP HEMT & 0.05 & 343 & 740 & {$[17]$} \\
\hline AlGaN/GaN-on-SiC HEMT & 0.1 & 153 & 230 & {$[18]$} \\
\hline AlGaN/GaN-on-Si HEMT & 0.5 & 32 & 27 & {$[19]$} \\
\hline
\end{tabular}

Table 2.2: Best reported $f_{T} \mathrm{~s}$ and $f_{\text {max }} \mathrm{s}$ for AlGaAs/GaAs, InP, and AlGaN/GaN HEMTs. 
novel technique is used, where an InGaN intermediate layer is grown on top of the GaN buffer layer on SiC, to enhance electron confinement in the 2DEG, which effectively increases the $f_{T}$ and $f_{\text {max }}$ to record levels of $153 \mathrm{GHz}$ and $230 \mathrm{GHz}$, respectively [18]. In spite of the advances made in fabricating GaN-based devices on $\mathrm{Si}$, GaN-on-SiC HEMTs still offer comparatively superior high-speed performance but at a much higher cost.

Table 2.3 compares some of the best reported power densities for AlGaAs/GaAs, InP, and $\mathrm{AlGaN} / \mathrm{GaN}$ devices. InP HEMTs are most effective at higher frequencies (V-band

\begin{tabular}{|c|c|c|c|c|c|}
\hline Transistor & $f[\mathrm{GHz}]$ & $P_{\text {out }}[\mathrm{W}]$ & $P A E[\%]$ & $P_{\text {density }}[\mathrm{W} / \mathrm{mm}]$ & Ref. \\
\hline \hline AlGaAs/GaAs HEMT & 2.2 & 40 & 35 & 0.37 & {$[20]$} \\
\hline InP HEMT & 60 & 0.186 & 36 & 0.37 & {$[21]$} \\
\hline AlGaN/GaN-on-SiC HEMT & 4 & 8 & 54.8 & 32.2 & {$[22]$} \\
\hline AlGaN/GaN-on-Si HEMT & 2.14 & 1.2 & 52.7 & 12 & {$[23]$} \\
\hline
\end{tabular}

Table 2.3: Best reported power performances of AlGaAs/GaAs, InP, and AlGaN/GaN HEMTs.

and W-band), so much of the recent literature reports power densities for frequencies in the millimeter-wave region of the spectrum. The first reporting of the InP HEMT's power capability demonstrates $288 \mathrm{~mW}$ of output power with $40 \%$ PAE and a power density of $0.96 \mathrm{~W} / \mathrm{mm}$ [24]. InP devices are well-known for their superior channel transport properties and low breakdown voltage. In general, the low breakdown field level is a direct consequence of the In content in the channel [25]. The breakdown levels in both GaAs- and InP-based devices are a major concern, especially as mobile services migrate toward higher frequencies.

Compared to all other substrate materials, silicon is the most highly refined bulk substrate known to exist; it is free of intrinsic defects, it is more thermally conductive 
than GaAs, and it is lower in cost than both $\mathrm{SiC}$ and GaAs. With $f_{T} \mathrm{~s}$ above $30 \mathrm{GHz}$, GaN-on-Si HEMTs are still an attractive solution, both in terms of performance and cost, to meet the power and efficiency needs of next-generation microwave base station systems. 


\section{Chapter 3}

\section{Microwave Power Amplifiers}

Power amplifiers generally exist in either the current source mode, where the transistor serves as a high-impedance current source, or the switched mode, where the transistor acts as an on/off switch. This chapter aims to illustrate the major differences between conventional (eg. Class A, Class AB, Class B, and Class C) PAs and the Class E switchedmode topology. In doing so, generalized analyses of both current source and Class E amplifiers are presented accounting for device parasitics that impose practical limits on performance. Small- and large-signal stability concepts are discussed. The chapter concludes with a brief examination of prior art in demonstrated Class E PAs in AlGaN/GaN HEMT technology. Power amplifier specifications, that form the basis for the subsequent chapters, are outlined as well.

\subsection{On Power, Bandwidth, and Linearity}

Several well respected texts make reference to power amplifiers that transmit low, medium, or high power, but in a vaguely defined way $[26,27]$. The same ambiguity is also commonly 
found with respect to narrowband and wideband operation. In the context of this thesis, "narrowband" implies that the frequency bandwidth is less than one octave and "low frequencies" are in the L-band or lower. "Low power" refers to RF output powers below $500 \mathrm{~mW}$, power amplifiers that transmit in the range from $0.5 \mathrm{~W}$ to $1 \mathrm{~W}$ are categorized as medium power, and high power amplifiers are those that produce RF power levels in excess of $1 \mathrm{~W}$.

All large-signal power amplifiers, whether operating in the current-source mode or switched mode, exhibit nonlinear behaviour. This holds true, even for the familiar Class A topology, whose operation is quasi-linear, at best [26]. Linearity is, by definition, a measure of how well the amplitude of the output signal corresponds to that of the input signal, where the output amplitude is greater than the input amplitude by a factor equal to the gain of the power amplifier. As discussed in Section 3.3, Class E power amplifiers, in their basic form and by nature of their design, are inherently nonlinear and theoretically achieve $100 \%$ drain efficiency. Linearity or linearization schemes are outside the scope of this work and are not discussed in any detail here.

\subsection{Conventional Power Amplifiers}

Traditional power amplifiers are classed according to the fraction of the signal's natural period that the transistor conducts current. Figure 3.1 shows a generic set of DC $I_{d s}$ vs. $V_{d s}$ curves superimposed by load-lines for the four main conventional PA classes. Also shown are the corresponding drain current segments illustrating how the drain current waveform is shaped by the class of operation. The Class A power amplifier, with its $100 \%$ 


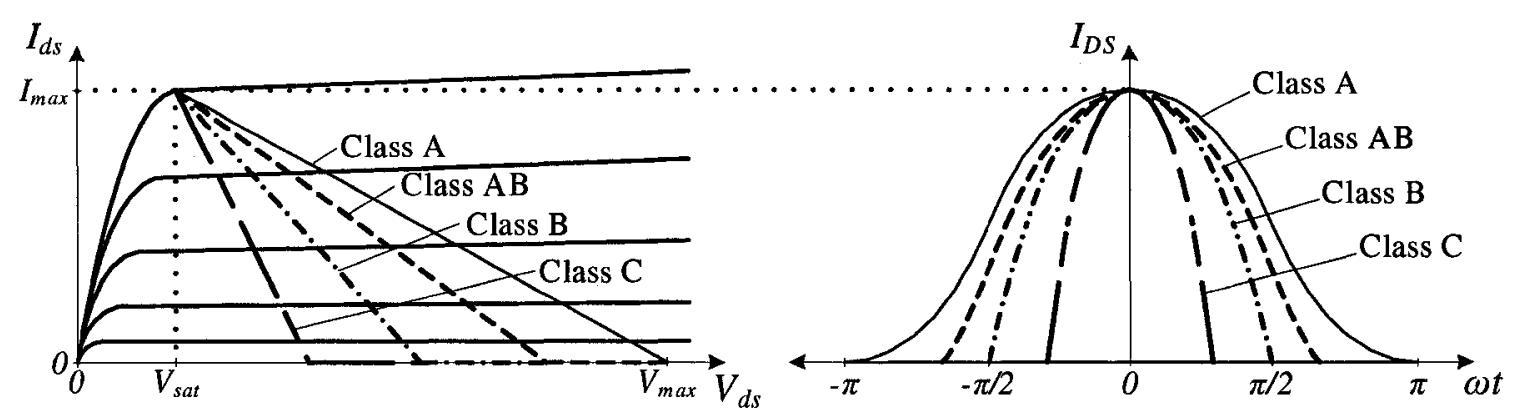

Figure 3.1: Generic DC $I_{d s}$ vs. $V_{d s}$ curves, load-lines, and drain current profiles for conventional PA configurations.

duty-cycle, supports continuous-wave drain current and is consequently the least efficient. Power amplifier efficiency progressively increases in the Class AB, Class B, and Class C configurations, as the biasing and drain loading conditions are adjusted to shorten the duty cycle. The drain current assumes the shape of a pulsed waveform, reducing the fraction of time that the transistor conducts current (or, the conduction angle). Decreasing the conduction angle also reduces the amount of time that the transistor spends dissipating power.

The four traditional power amplifiers may be analyzed using the power amplifier model shown in Figure 3.2 and the waveforms shown in Figure 3.3. The circuit consists of ideal lumped inductors and capacitors; bias is inserted through ideal RF chokes (RFCs) and the desired signal is coupled to the load through ideal DC blocking capacitors. The high-Q harmonic trap included at the output of the circuit presents the short-circuit condition for harmonic currents so that the drain voltage remains sinusoidal and only the fundamental frequency signal appears at the load. Detailed analyses of the circuit in Figure 3.2 may be found in several excellent texts on the topic [26-29], so only the most relevant results are presented here. The following analysis assumes that $V_{s a t}$ in Figure 3.1 is zero. Also 


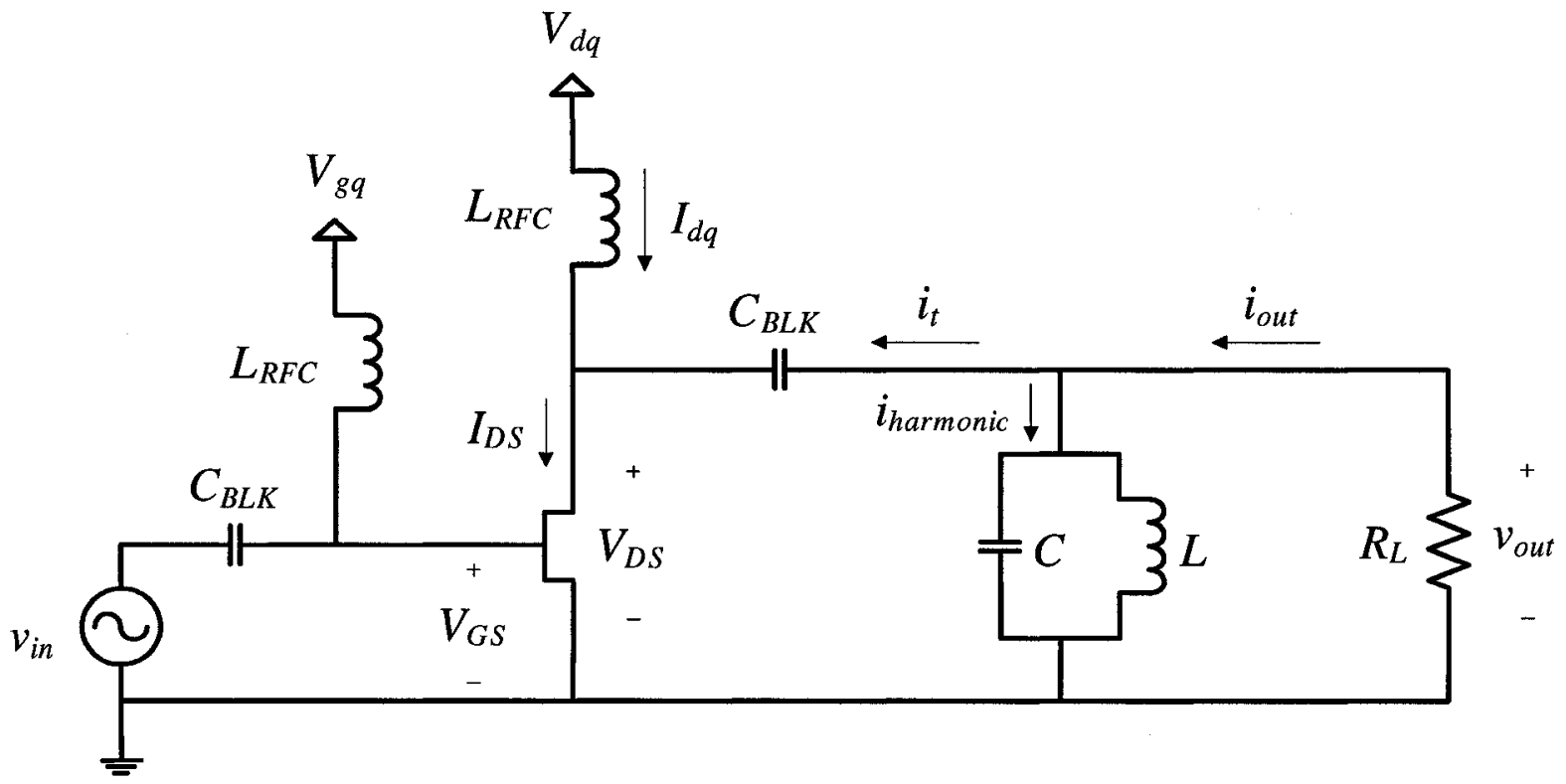

Figure 3.2: Generic power amplifier schematic. The high-Q harmonic trap ensures a pure sinusoidal output voltage.

note that the $I_{d s^{-}}-V_{g s}$ response in Figure 3.3 is a standard piecewise-linear approximation of the actual nonlinear characteristic.

Referring to Figure 3.2, the gate and drain voltages are

$$
\begin{gathered}
V_{G S}=V_{g q}+v_{g s}=V_{g q}+v_{i n} \\
V_{D S}=V_{d q}+v_{o u t}
\end{gathered}
$$

where

$$
v_{\text {out }}=-i_{\text {out }} R_{L}
$$

Expanding Equations 3.2.1 and 3.2.2 to include the cosinusoidal input and output voltages gives

$$
\begin{gathered}
V_{G S}=V_{g q}+V_{\text {in }} \cos (\omega t) \\
V_{D S}=V_{d q}-V_{o u t} \cos (\omega t)
\end{gathered}
$$




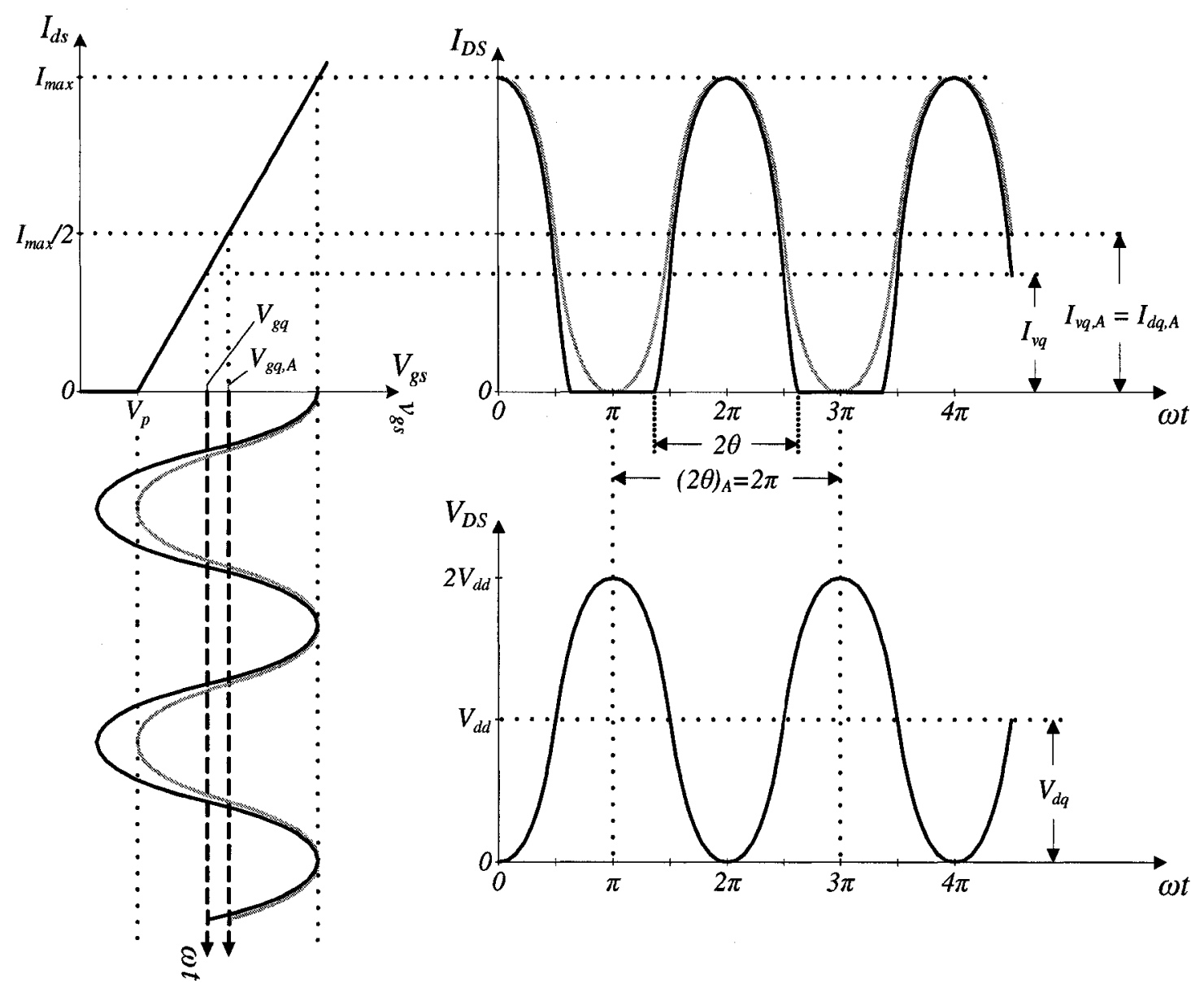

Figure 3.3: Generic PA voltage and current waveforms. Variables denoted with the 'A' subscript correspond to Class A quantities. 
The drain bias current in Figure 3.3 has been defined as a fictitious virtual current, $I_{v q}$, in order to simplify the drain current derivation. As shown in Figure 3.3, as $I_{v q}$ is lowered beyond the level required for Class A operation, the cycle over which conduction occurs

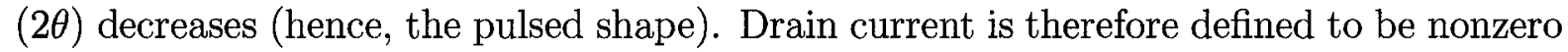
only over the region of conduction:

$$
I_{D S}=\left\{\begin{array}{cc}
I_{v q}+I_{o u t} \cos (\omega t) & -\theta \leqslant \omega t<\theta \\
0 & \theta \leqslant \omega t<2 \pi-\theta
\end{array}\right.
$$

The virtual bias current can be derived using Equation 3.2.6 and by setting $I_{D S}$ to zero when $\omega t=\theta$ :

$$
I_{v q}=-I_{o u t} \cos \theta
$$

Note that $I_{d q}$ is the actual DC current drawn from the drain power supply. Indeed, $I_{d q}$ decreases as $I_{v q}$ decreases but $I_{d q}$ is the average current required from the power supply for pulsed current operation, whereas $I_{v q}$ is simply an offset so the drain current shown in Equation 3.2.6 and Figure 3.3 can be expressed as a DC component superimposed by a perfect sinusoid. Only in the special case of a continuously conducting Class A power amplifier (where the conduction angle is $2 \pi$ ), is the virtual bias current equivalent to $I_{d q}$. It follows then, that mathematically, the total drain current, $I_{D S}$, is

$$
I_{D S}=I_{0}+I_{1} \cos (\omega t)+I_{2} \cos (2 \omega t)+I_{3} \cos (3 \omega t)+\ldots
$$

where the DC and fundamental components, $I_{0}$ and $I_{1}$, respectively, are functions of the conduction angle:

$$
I_{0}=\frac{I_{\text {out }}}{\pi}(\sin \theta-\theta \cos \theta)
$$




$$
I_{1}=\frac{I_{\text {out }}}{\pi}(\theta-\sin \theta \cos \theta)
$$

The well-known expression for drain efficiency $(D E)$ is easily obtained by taking the ratio of the DC and RF power levels ( $P_{0}$ and $P_{1}$, respectively) obtained using Equations 3.2 .9 and 3.2.10. The DC output power, the power at the fundamental, and the drain efficiency can be written as

$$
\begin{gathered}
P_{0}=\frac{I_{\text {out }} V_{d q}}{\pi}(\sin \theta-\theta \cos \theta) \\
P_{1}=\frac{I_{\text {out }} V_{\text {out }}}{2 \pi}(\theta-\sin \theta \cos \theta) \\
D E=\frac{1}{2} \frac{V_{\text {out }}}{V_{d q}} \frac{\theta-\sin \theta \cos \theta}{\sin \theta-\theta \cos \theta}
\end{gathered}
$$

where, in the ideal case, $V_{\text {out }}=V_{d q}$. Another measure of efficiency, the power-added efficiency $(P A E)$, factors the gain of the PA into the calculation:

$$
P A E=\frac{P_{1}-P_{A V S}}{P_{0}}
$$

where $P_{A V S}$ is the power available from the source.

It is clear from the above analysis that, not only is the Class A power amplifier the least efficient $\left(D E_{\max , A}=50 \%\right)$, but it has zero efficiency when no input power is applied. The other current source topologies attempt to increase power amplifier efficiency by reducing the conduction angle and modifying the loading conditions such that DC power is dissipated in the transistor only during conduction. In contrast to the high linearity and gain offered by the Class A power amplifier, the pulsed current topologies require larger input drive signals forcing the degradation of both gain and linearity for higher efficiency levels. Table 3.1 shows the conduction angles and the theoretical maximum drain efficiencies for the conventional amplifiers discussed so far. 


\begin{tabular}{|c|c|c|}
\hline PA Class & Conduction Angle $\left.{ }^{\circ}\right]$ & $D E_{\max }[\%]$ \\
\hline \hline $\mathrm{A}$ & 360 & 50 \\
\hline $\mathrm{AB}$ & $360-180$ & $50-78.5$ \\
\hline $\mathrm{B}$ & 180 & 78.5 \\
\hline $\mathrm{C}$ & $180-0$ & $78.5-100$ \\
\hline
\end{tabular}

Table 3.1: Conduction angles and maximum theoretical drain efficiencies for conventional PAs.

The preceding analysis has maintained ideal conditions, where it has been assumed that $V_{\text {sat }}=0$ and the output voltage amplitude is equal to the drain bias level. Realistically however, $V_{d q}, V_{\text {out }}$, and the voltage swing across the device are constrained by a nonzero $V_{\text {sat }}$ at the knee and $V_{\max }$, which is typically near the drain-source breakdown voltage (see Figure 3.1). It follows that

$$
\begin{aligned}
& V_{\text {out }, \text { practical }}=\frac{V_{\text {max }}-V_{s a t}}{2} \\
& V_{d q, \text { practical }}=\frac{V_{\text {max }}+V_{s a t}}{2}
\end{aligned}
$$

Substituting Equations 3.2.15 and 3.2.16 into Equations 3.2.12 and 3.2.14 gives practical expressions for fundamental power and drain efficiency:

$$
\begin{gathered}
P_{1, \text { practical }}=\frac{I_{\text {out }}\left(V_{\text {max }}-V_{\text {sat }}\right)}{4 \pi}(\theta-\sin \theta \cos \theta) \\
D E_{\text {practical }}=\frac{1}{2} \frac{V_{\text {max }}-V_{\text {sat }}}{V_{\text {max }}+V_{\text {sat }}} \frac{\theta-\sin \theta \cos \theta}{\sin \theta-\theta \cos \theta}
\end{gathered}
$$

Implicit in the design equations is the assumption that the transistor behaves as a high-impedance current source. The drain current amplitude and the loading conditions are fine-tuned to ensure that the drain voltage swing is just large enough to bring the minimum $V_{D S}$ to $V_{\text {sat }}$ without causing the device to enter the low-impedance triode region. Choosing a device that has a high breakdown voltage and low $V_{\text {sat }}$ are two key requirements 
for medium to high power, high efficiency operation. Regardless of which current source amplifier topology is chosen, $V_{\text {sat }}$ will always be a major source of power dissipation in the device. Also, factors such as device heating influence practical efficiencies: in some cases, up to ten times more DC power goes into a system than the RF level received at the transmitting antenna [30].

\subsection{Class E Switched-Mode Power Amplifier}

The Class E switched mode power amplifier, originally conceived by the Sokals [31], operates under the premise that the transistor behaves as a discrete on/off switch with ideally zero on-state and infinite off-state resistances. Figure 3.4 shows an ideal Class E PA circuit. The circuit is essentially a power converter, whose fundamental output signal amplitude is determined by the drain bias supply voltage and the load resistance. The output signal is only weakly correlated to the input signal in that the input drive must be large enough to adequately switch the transistor between the fully on and fully off states. Variable envelope input signals, such as those used in amplitude modulated or single side-band broadcasting, cannot be used with the expectation that the information in the envelope will be maintained at the output. This limitation forces a constant-envelope output signal making the amplifier nonlinear, as discussed in Section 3.3.1. Proper load network design at the output of the transistor prohibits the simultaneous occurrence of high voltage and high current, allowing the amplifier to operate with $100 \%$ theoretical drain efficiency. 
Much of the published work on the Class E mode assumes ideal transistor switching conditions and carries these assumptions through to design and implementation [32]. While there exist active devices that approximate switching behaviour quite well at frequencies below $1 \mathrm{GHz}$, nonlinear models must be used at higher frequencies where transistors no longer behave as simple switches. This chapter starts with the basic theory behind the original Class E circuit assuming ideal conditions and ends with a discussion on practical issues that degrade amplifier performance at microwave frequencies.

\subsubsection{Idealized Analysis}

The original form of the shunt capacitance Class E circuit is the focus of this work. This configuration is easily manipulated to accommodate transmission line loading at microwave frequencies - a requirement for the planar microwave power amplifier in Chapter 5. Figure 3.4 depicts an illustration of the ideal circuit with a capacitor, $C_{P}$, shunting a single-pole, single-throw switch. The corresponding voltage and current waveforms are shown in Figure 3.5. $C_{P}$ is considered a linear design parameter whose value is analytically computed. In actual fact, that capacitor is usually entirely (or at least partially) composed of the output capacitance of the active device. Equally important is the series resonator that consists of $L_{S}$ and $C_{S}$ and is tuned to the fundamental frequency, $f_{1}{ }^{1}$. Several assumptions about the basic circuit are made to simplify the analysis:

- The switch that models the transistor is lossless and has instantaneous switching action.

\footnotetext{
${ }^{1}$ Note that $f_{1}$ and $f_{s}$ are one in the same.
} 


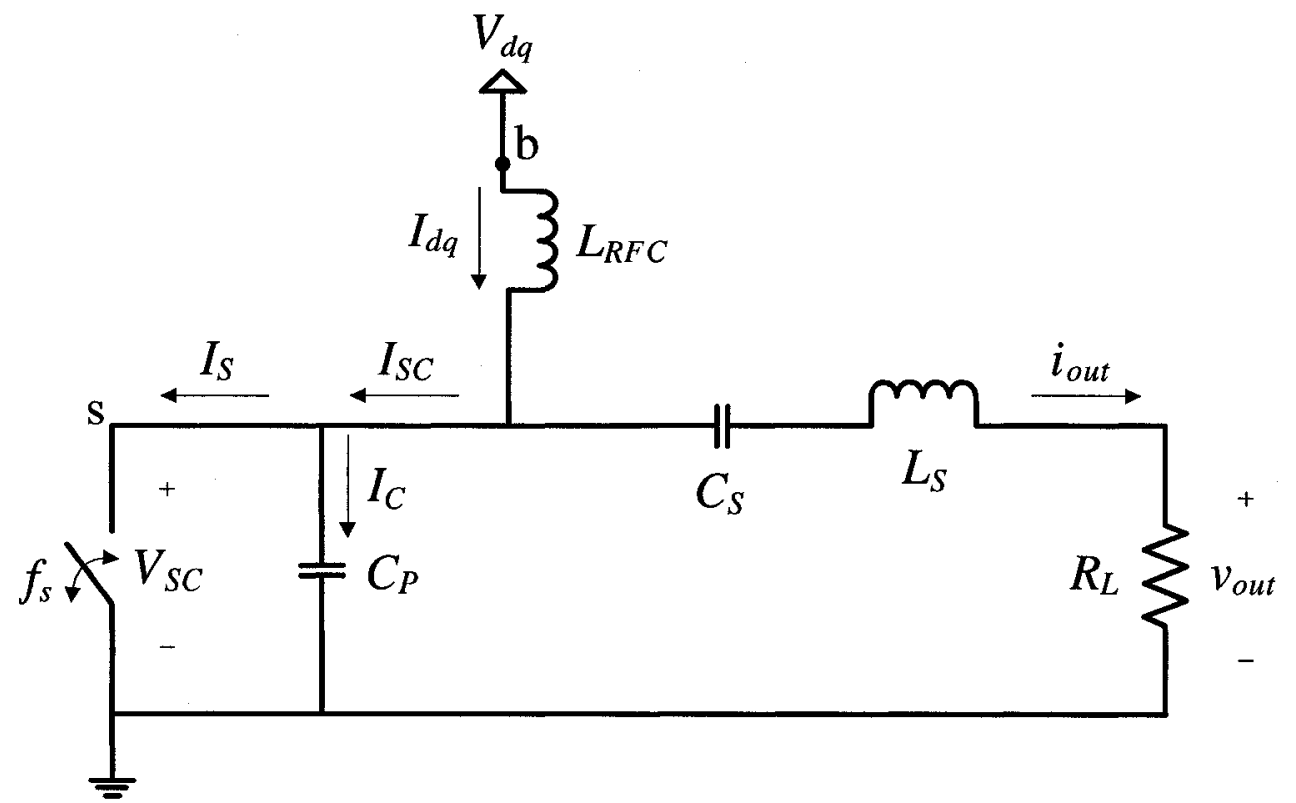

Figure 3.4: Ideal Class $\mathrm{E}$ power amplifier circuit. The ideal single-pole, single-throw switch transitions states at the switching frequency, $f_{s}$. The PA generates RF output power also at $f_{s}$.

- The inductor through which the DC bias is supplied is lossless and has a reactance that is high enough to not load the circuit and to disallow any variations in DC current.

- The series resonant circuit has a sufficiently high Q-factor to force a sinusoidal output current at the fundamental (or switching) frequency.

Consider the voltage and current waveforms in Figure 3.5. The fact that nonzero voltage and current never coexist is a direct consequence of using an ideal switch as the active element. The Class E concept is defined by three principle objectives that fulfill the above condition $[33,34]$ :

- When the switch opens, the drain voltage $\left(V_{S C}\right)$ should not rise until the transistor 


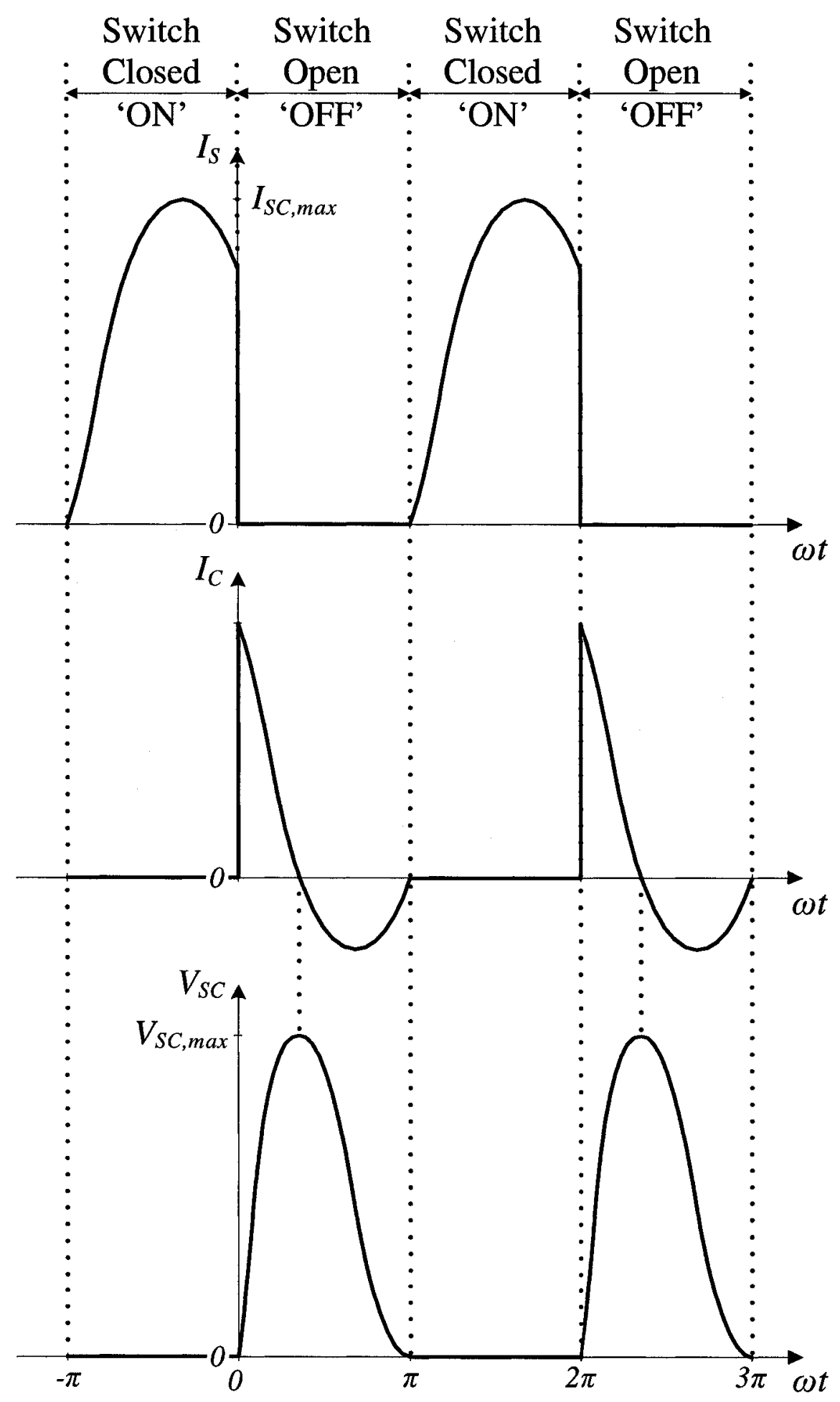

Figure 3.5: Ideal Class E PA voltage and current waveforms 
is completely off $\left(I_{S}=0 \mathrm{~A}\right)^{2}$

- When the switch closes, the drain voltage $\left(V_{S C}\right)$ should be zero before the drain current begins to rise.

- When the switch closes, the slope of the drain voltage should be zero.

In his paper [34], Raab concludes that optimal Class E operation is achieved with a $50 \%$ duty cycle switching period and re-affirms Sokal's third principle objective listed above. Under these conditions, the load network must provide a phase shift $(\varphi)$ of approximately $-32^{\circ}$ to the output current. He represents this phase shift as a fictitious reactance (that turns out to be inductive), whose value is simply the difference in reactances of the inductor and capacitor in the resonant circuit. The analysis that follows is loosely based on [34]; a slightly different approach is presented in [35].

The circuit analysis, referring to Figure 3.4, continues with the foresight that the output current waveform is nominally phase shifted. Then the current through the switchcapacitor combination is

$$
I_{S C}=I_{d q}-I_{o u t} \sin (\omega t+\varphi)
$$

keeping in mind that $I_{S C}$ flows through the switch when it is closed and through $C_{P}$ when it is open. The voltage at node 's' is determined by the charging of $C_{P}$ when the switch is off:

$$
V_{S C}=\frac{1}{\omega C_{p}} \int_{0}^{\omega t} I_{C} d(\omega t)
$$

\footnotetext{
${ }^{2}$ For the purpose of the ideal circuit explanation, drain voltage is denoted $V_{S C}$ and drain current is $I_{S}$. These symbols are replaced with transistor quantities in subsequent chapters
} 


$$
\begin{aligned}
V_{S C} & =\frac{1}{\omega C_{p}} \int_{0}^{\omega t}\left[I_{d q}-I_{o u t} \sin (\omega t+\varphi)\right] d(\omega t) \\
V_{S C} & =\frac{V_{o u t}}{\omega C_{p} R_{L}}[\cos (\omega t+\varphi)-\cos \varphi]+\frac{I_{d q} \omega t}{\omega C_{p}}
\end{aligned}
$$

Equation 3.3.4 has been simplified given a $50 \%$ duty cycle and zero drain voltage slope at switch turn on. Based on these optimum conditions, $V_{\text {out }}$ can be expressed in terms of $V_{d q}$

$$
V_{\text {out }}=\frac{2 V_{d q}}{\sqrt{1+\frac{\pi^{2}}{4}}}
$$

The drain bias current, $I_{d q}$, is related to $V_{d q}$ by the resistive load, $R_{d c}$, presented by the amplifier to the bias terminals at node ' $\mathrm{b}$ ':

$$
V_{d q}=I_{d q} R_{d c}
$$

The resistance, $R_{d c}$, also being a function of duty cycle, is

$$
R_{d c}=\frac{\left(1+\frac{\pi^{2}}{4}\right) R_{L}}{2}
$$

for a $50 \%$ duty cycle.

Since every element in the circuit, except for $R_{L}$, is lossless, there is no DC power dissipated as heat - all of it is converted to useful RF energy that reaches the load. The DC input power and the RF output power are

$$
\begin{gathered}
P_{0}=\frac{V_{d q}^{2}}{R_{d c}} \\
P_{1}=\frac{1}{2} \frac{V_{o u t}^{2}}{R_{L}}
\end{gathered}
$$

Substituting Equation 3.3.7 into Equation 3.3.8 and Equation 3.3.5 into Equation 3.3.9 gives Equations 3.3.10

$$
P_{0}=P_{1}=\frac{2 V_{d q}^{2}}{\left(1+\frac{\pi^{2}}{4}\right) R_{L}}
$$


For the sake of completeness, taking the ratio of $P_{1}$ to $P_{0}$ gives a drain efficiency, $D E=$ $100 \%$.

It is noteworthy, at this point, to emphasize the importance of choosing the right device technology. If the goal is to maximize output power (i.e. optimum Class E operation), then peak drain voltage and current excursions must be known during device selection. In his analysis, Raab derives a relationship between $R_{d c}, \omega$, and $C_{P}$ which, for a $50 \%$ duty cycle, is

$$
\omega C_{p} R_{L}=\frac{-\tan \varphi}{2}
$$

Substitution of Equations 3.3.5, 3.3.6, 3.3.7, and 3.3.11 into Equation 3.3.4 yields

$$
V_{S C}=V_{d q}\left\{\frac{-4}{\tan \varphi} \sqrt{1+\frac{\pi^{2}}{4}}[\cos (\omega t+\varphi)-\cos \varphi]-\frac{2 \omega t}{\tan \varphi}\right\}
$$

Differentiating Equation 3.3.12 with respect to $\omega t$ and setting the result to zero gives the time at which the drain voltage is at its maximum for a given $V_{d q}$ :

$$
(\omega t)_{V_{S C, \max }}=\sin \left(\frac{1}{\sqrt{1+\frac{\pi^{2}}{4}}}\right)-\varphi
$$

Substituting Equation 3.3.13 and the known phase angle, $\varphi=-0.5669 \mathrm{rad}$, into Equation 3.3.12 gives the peak drain voltage,

$$
V_{S C, \max }=3.562 V_{d q}
$$

Maximum drain current is easily determined from Equation 3.3.1 by observing that

$$
I_{S C, \max }=I_{d q}+\frac{V_{o u t}}{R_{L}}
$$


Successive substitutions of Equation 3.3.5 followed by Equation 3.3.6 into Equation 3.3.15 gives

$$
I_{S C, \max }=2.862 I_{d q}
$$

The physical nature of the AlGaN/GaN heterostructure permits high sheet charge density in the conducting 2DEG and allows the device to withstand a high critical electric field. The AlGaN/GaN HEMT can therefore sustain high voltages whilst generating high RF current, making it an ideal choice for this work.

The beauty of the Class E PA lies in its complete end-to-end realizability. In general, specific design equations can be used to build a first-time successful, fully operational Class E amplifier [33]. Although this last statement is true, it only really applies to the ideal situation or in the low-frequency case where parasitic effects can be (mostly) ignored. At microwave frequencies device parasitics have to be included in the design.

\subsubsection{The Class E Concept at GHz Frequencies}

The key difference between conventional power amplifiers and the Class E amplifier is the range of allowable voltages that exist across the drain and source terminals of the device. Particularly important is the effect of the transistor's knee voltage on achieving high efficiency operation. During conduction, the device never enters the triode region when operated as a high-impedance current source; but when operated as a switch, the drain-source voltage must be as low as possible requiring traversal into the triode region during part of the RF cycle. The actual efficiency reduction, in practice, is attributed to the combined power dissipated in this finite on-resistance as well as in the drain and source 
resistances, $R_{s}$ and $R_{d}$ [36]. The microwave power amplifier in Chapter 5 is implemented with transmission lines in order to reduce insertion loss. This design strategy alone prevents drain efficiency from reaching $100 \%$ since optimal open-circuit loading is rarely achieved at all higher-order harmonics of the fundamental frequency.

The notion that transistors can be replaced with simplified switch models for Class $\mathrm{E}$ PA designs is an unfortunate outcome of the ideal analysis. To quote Cripps, "RF power transistors are not switches at $\mathrm{GHz}$ frequencies, even if a finite on resistance is included in the switch model" [32]. Although the switch model is a good starting point in the design process, the final design must include a real large-signal transistor model that predicts parasitic and nonlinear effects at microwave frequencies. This is especially true for this work since a $3.5 \mathrm{GHz}$ square-wave input drive is not realistic; instead, a large-amplitude voltage sinusoid is used to switch the device.

So far, the Class E PA has been discussed under the assumption that the transistor only switches between two discrete states: on and off. Implicit in the assumption is that the gate of the transistor is driven with an ideal square-wave. Driving circuits alone are a topic worthy of a thesis and so are not discussed here; Sokal [33] and Ortega-Gonzalez [37] cover gate driving circuits and methods in their respective articles. It is common practice to over-drive the input (i.e. the gate of the active device) of a switching PA with a large-amplitude sinusoidal voltage in the absence of a driving circuit or, in this case, the unavailability of a high frequency square-wave generator. The over-driving technique is used in this work with the expectation of large energy losses and reductions in gain. The sinusoidal driving power required to completely switch the device $\left(P_{S W}\right)$ is a function of 
the gate-source voltage needed to switch the gate $\left(V_{g s w}\right)$, the input capacitance $\left(C_{g s}\right)$, and the switching frequency. Equation 3.3.17 embodies all of these parameters [37].

$$
P_{S W}=C_{g s} V_{g s w}^{2} f
$$

As a final thought when choosing the right device technology, one must bear in mind that parasitic capacitances invariably pose an upper limit on the frequency permissible for Class E operation. A good approximation for this maximum frequency is derived in [38] and reproduced here,

$$
f_{\max , E}=\frac{I_{\max }}{56.5 C_{d s o} V_{d q}}
$$

noting that $I_{\max }$ equates to the maximum current-handling capability of the active device switch, which is ideally $I_{S C, \max }$ from Equation 3.3.16. $C_{d s o}$ is the output capacitance of the transistor.

\subsection{Stability}

Almost all RF power transistors are potentially unstable. That is, the transistor may oscillate for some source and load impedances that present negative resistances to the terminals of the transistor. But negative resistances do not guarantee oscillations; the terminations must present the correct phase and magnitude to make the transistor oscillate [32]. The stability of a device is determined by the Rollet stability factor, $\mathrm{k}$,

$$
k=\frac{2 \Re\left(Y_{11}\right) \Re\left(Y_{22}\right)-\Re\left(Y_{12} Y_{21}\right)}{\left|Y_{12} Y_{21}\right|}
$$


where $k>1$ for unconditional stability and $k<1$ for conditional stability [39]. Strictly speaking, the stability criterion is for the small-signal case and does not accurately represent the stability performance of a nonlinear power amplifier. Moreover, a Class $\mathrm{E}$ power amplifier is biased in the off state (i.e. the gate is pinched-off), but large-signal variations at the input periodically bring the transistor into the on state. This switching behaviour is not captured in the evaluation of Equation 3.4.1. Small-signal stability is checked in the design, but is not relied upon as a definite measure of the PA's overall stability as a large-signal PA.

In large-signal power amplifiers, instability is more an artifact of the bias insertion circuit. Care is taken to ensure that the bias circuits do not present unwanted impedances to the power amplifier and that noise on the supply lines is suppressed so that it cannot couple into the gate or the drain of the transistor. Beyond that, the problem of instability is tackled in the lab during testing. Oscillations typically appear as large variations in DC current and RF output power. An array of decoupling capacitors is used at the bias terminals to suppress potential oscillations from low frequencies to frequencies beyond the operating frequency of the PA.

\subsection{Previous Work and Proposed Amplifier Specifi- cations}

In spite of its relative infancy in GaN electronics, the switched-mode Class-E concept, using GaN-on-SiC HEMTs, has been demonstrated for a range of applications from low frequency, high-voltage power electronics to microwave power amplification [40-43]. At 
microwave frequencies, $\mathrm{Xu}$ [42] and Gao [43] report monolithic GaN-on-SiC Class-E implementations at $1.9 \mathrm{GHz}$ and $2.0 \mathrm{GHz}$, both having similar output powers and $P A E \mathrm{~s}$ of $37 \mathrm{dBm}$ at $57 \%$ PAE. Cree $^{3}$ holds the current world record for the highest demonstrated $P A E$ of $84 \%$ and output power of $10 \mathrm{~W}$ at $2 \mathrm{GHz}$ [44]. These designs employ expensive SiC-based GaN devices and require higher drain bias levels (greater than $20 \mathrm{~V}$ ) to achieve the reported results. The relatively few reports of GaN-based Class E PAs shows the need for further study of GaN-based switched-mode PA topologies.

This work demonstrates the first reported Class E power amplifier using GaN HEMTs fabricated on silicon. The amplifier is designed for operation at $3.5 \mathrm{GHz}$ in the WiMAX band. A standard $50 \mathrm{~W}$ bias supply, limited to $25 \mathrm{~V}$, is used for biasing. The drain of the HEMT is backed off from the supply limit down to $20 \mathrm{~V}$. The conservative choice of bias allows the drain voltage to swing to the required $71 \mathrm{~V}$ peak for ideal Class E operation and limits the output power to the theoretical maximum of $4 \mathrm{~W}$. In practice, neither the peak voltage nor the maximum output power is sustained as discussed in Chapter 5 . The gate of the HEMT is biased near the gate pinch-off voltage for optimal gain. These are the preliminary design specifications, as derived from the intended application and standard available biasing equipment; more complete specifications are given in Chapter 5.

\subsection{Summary}

The main difference between conventional and switched-mode PAs is in the regions of operation in which the active device is allowed to operate. For Class E operation, the

\footnotetext{
${ }^{3}$ Headquartered in North Carolina, Cree, Inc. designs and manufactures state-of-the-art GaN-on-SiC power HEMTs.
} 
transistor's $V_{D S}$ must approach as low an on-state voltage as possible (ideally $0 \mathrm{~V}$ ) for the half of the switching period when $I_{D S}>0 \mathrm{~A}$. When the transistor is off $\left(I_{D S}=0\right.$ A) for the other half of the period, $V_{D S}$ is permitted to traverse the horizontal $V_{d s}$ axis. Conventional PAs are required to operate in the saturation region at all times; that is, $V_{D S}$ may never swing into the triode region.

Depending on the class of operation (Class A, AB, B, or C), the time the transistor spends conducting current (or, the conduction angle) is determined by $V_{g s}$ and the output loading for a fixed $V_{d s}$ in the saturation region. As $V_{g s}$ is decreased towards the gate's pinch-off voltage, the conduction angle becomes shorter and the PA becomes more efficient. Theoretical maximum drain efficiencies range anywhere from $50 \%$ (Class A) to $100 \%$ (Class C). As the PA becomes more efficient, gain decreases and the region of input power levels over which gain remains constant becomes shorter. This efficiency vs. linearity trade-off is carried through to the Class C PA, where the gate of the transistor is biased below its pinch-off voltage, forcing the input drive amplitude to be larger than it would have to be for the same output power in a Class A PA.

The Class E PA relies on the active device behaving as an on/off switch, cycling through the low-impedance "on" and high-impedance "off" states at the fundamental frequency. Theoretical $100 \%$ drain efficiency is implied since, by definition, nonzero voltage and current never simultaneously exist across the terminals of a perfect switch. The output load network is tuned to ensure that $100 \%$ drain efficiency is maintained while the fundamental frequency signal is extracted at the output of the PA. While the Class E power amplifier efficiently generates power, the active device switch must support peak 
drain voltages over 3.5 times higher than the bias level and peak currents almost a factor of 3 higher than the average current drawn by the device. These constraints make GaN HEMTs attractive for application in switched-mode Class E PAs due to their ability to support high breakdown voltages and high current capacity.

A Class E PA targeting the WiMAX band at $3.5 \mathrm{GHz}$ with $4 \mathrm{~W}$ output power (based on a $20 \mathrm{~V}$ drain bias supply and $50 \Omega$ output load) is the focus of this work. Many factors such as, device parasitics, thermal effects, and transmission lines, influence circuit performance; in practice, both conventional and the Class E power amplifiers suffer from less than ideal efficiencies and output power levels. But generally speaking, the Class $\mathrm{E}$ PA out-performs conventional topologies in many aspects from circuit simplicity to output power and efficiency. 


\section{Chapter 4}

\section{Gallium Nitride High Electron Mobility Transistor Nonlinear Model}

An accurate large-signal (or nonlinear) transistor model provides the necessary foundation for many electronic designs. Device vendors don't always supply such models for even the most established technology. Transistor models are simply non-existent for the GaN HEMTs used in thesis.

Device models that are amenable to integration in circuit simulators are usually empirical in nature [45]. That is, the device model, although designed to represent the physical behaviour of a transistor, uses purely mathematical equations to approximate very complex operation as defined by measured data. The "best-fit" analytical equations are typically polynomials that have little physical significance to the device being characterized [46]. Indeed, physical models are more accurate, but difficult to implement in circuit simulators (see [47] and [48]) and data-based (eg. HP Root model) models are accurate only for the exact structures and measurement conditions under which the measurements were compiled. Between these two extremes are equation-based empirical 
models, which are most often found in circuit Computer-Aided Design (CAD) tools. The reader is referred to [49] and [50] for an overview of the most popular nonlinear device models by Materka et al. [51], Curtice et al. [52], and Statz et al. [53].

GaN HEMT technology is still a newcomer to the device modeling community, so largesignal models are not readily available. A nonlinear model originally conceived for GaAs HEMTs is used for this thesis due to the structural similarities between AlGaAs/GaAs AlGaN/GaN transistors. Model imperfections surface in the course of device characterization targeting physical properties of the GaN HEMT that are difficult to predict by the chosen model.

This chapter explores the versatility of the EEsof Scalable Nonlinear HEMT (EEHEMT) model offered as an integrated component in the Advanced Design System (ADS) RF circuit simulator [54]. The flexibility of this model lies in its ability to be molded to fit the electrical behaviour of most HEMTs using measured DC and RF data. This model is chosen, in part, due to its straightforward manipulation given the types of data supplied by the device manufacturer, and also as an exploratory exercise to determine the areas of the model that need modification in order to accommodate emerging GaN technology.

The chapter begins with an introduction to the GaN-on-Si HEMT used in this work. A discussion on measurement methods and parameter extraction follows a review of the specifics of the nonlinear model. Small- and large-signal characterization is presented leading to the final nonlinear model, which approximately fits DC, transconductance, and s-parameter data with reasonable accuracy for the Class E power amplifier design presented in Chapter 5. The model is not perfect: there are regions of operation where 
the simulated vs. measured performance deviates enough to warrant a brief examination towards the end of the chapter.

\subsection{The Nitronex GaN-on-Si HEMT}

The Nitronex GaN-on-Si HEMT technology is based on the proprietary SIGANTIC@ and PENDEO $^{\mathrm{TM}}$ processes that allow high-quality GaN crystal growth on silicon substrates [55]. The GaN HEMT used in this work is an unpackaged bare die with gold-plated gate, drain, and source contacts that are attached, via bond wires, to the microwave circuit elements of the Class E power amplifier.

The top-level layout of the GaN-on-Si HEMT is shown in Figure 4.1 with typical device properties summarized in Table 4.1. The performance data, collected at $2.14 \mathrm{GHz}$,

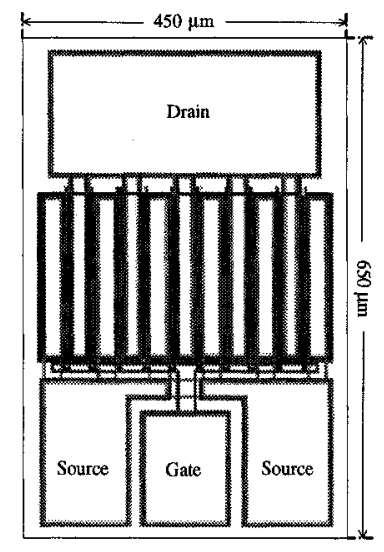

Figure 4.1: Top-level layout of the $\mathrm{Ni}$ tronex GaN-on-Si HEMT die showing a gate width of $10 \times 0.2 \mathrm{~mm}$.

\begin{tabular}{|c|c|}
\hline Gate periphery & $2 \mathrm{~mm}$ \\
Channel length & $0.7 \mu \mathrm{m}$ \\
$f_{T}$ & $13 \mathrm{GHz}$ \\
\hline Drain bias supply & $15 \mathrm{~V}$ \\
Quiescent drain current & $450 \mathrm{~mA}$ \\
Output power & $4-5 \mathrm{~W}$ \\
Peak drain efficiency & $60-65 \%$ \\
Linear gain & $18-19 \mathrm{~dB}$ \\
\hline
\end{tabular}

Table 4.1: Vendor-supplied GaN-on-Si HEMT characteristics at $2.14 \mathrm{GHz}$

was supplied by the device manufacturer.

Each GaN HEMT was cut out of a larger die, containing several test structures. The physical dimensions shown in the figure include tolerances added to accommodate dicing. 


\subsection{EEsof $2 \mathrm{~mm}$ Nonlinear HEMT Model}

The EEHEMT model fits analytic expressions to measured DC and bias-dependent sparameter data. The model as a whole is unique, but its individual components are not in that they are borrowed from the works presented in [45], [56], [57], and [53]. Like many of the more popular models, the EEHEMT model's fitting parameters allow it to approximate the operation of many different process types. On the downside, the model's analytic expressions are not accessible for modification by the user, causing potential inaccuracies in the simulated behaviour of the device.

The EEHEMT model includes simple linear scaling for designing with larger periphery or multiple devices in parallel. The model also characterizes resistor and channel noise and includes a means by which to simulate the design at temperatures other than the temperature at which the equivalent circuit parameters are extracted. The model is comprehensive enough to address a variety of uses, but at the same time allows the designer to use only those features that are specific to one particular application. In that regard, the GaN transistors used in this thesis have a $2 \mathrm{~mm}$ gate periphery and are exclusively used in power amplifier applications, where the ambient air is at room temperature, so the gate width and temperature scaling features and the channel noise model are not used.

The schematic representation of the model, shown in Figure 4.2, consists of some notable features that are summarized in the following sections. The complete documentation can be found in [54]. 


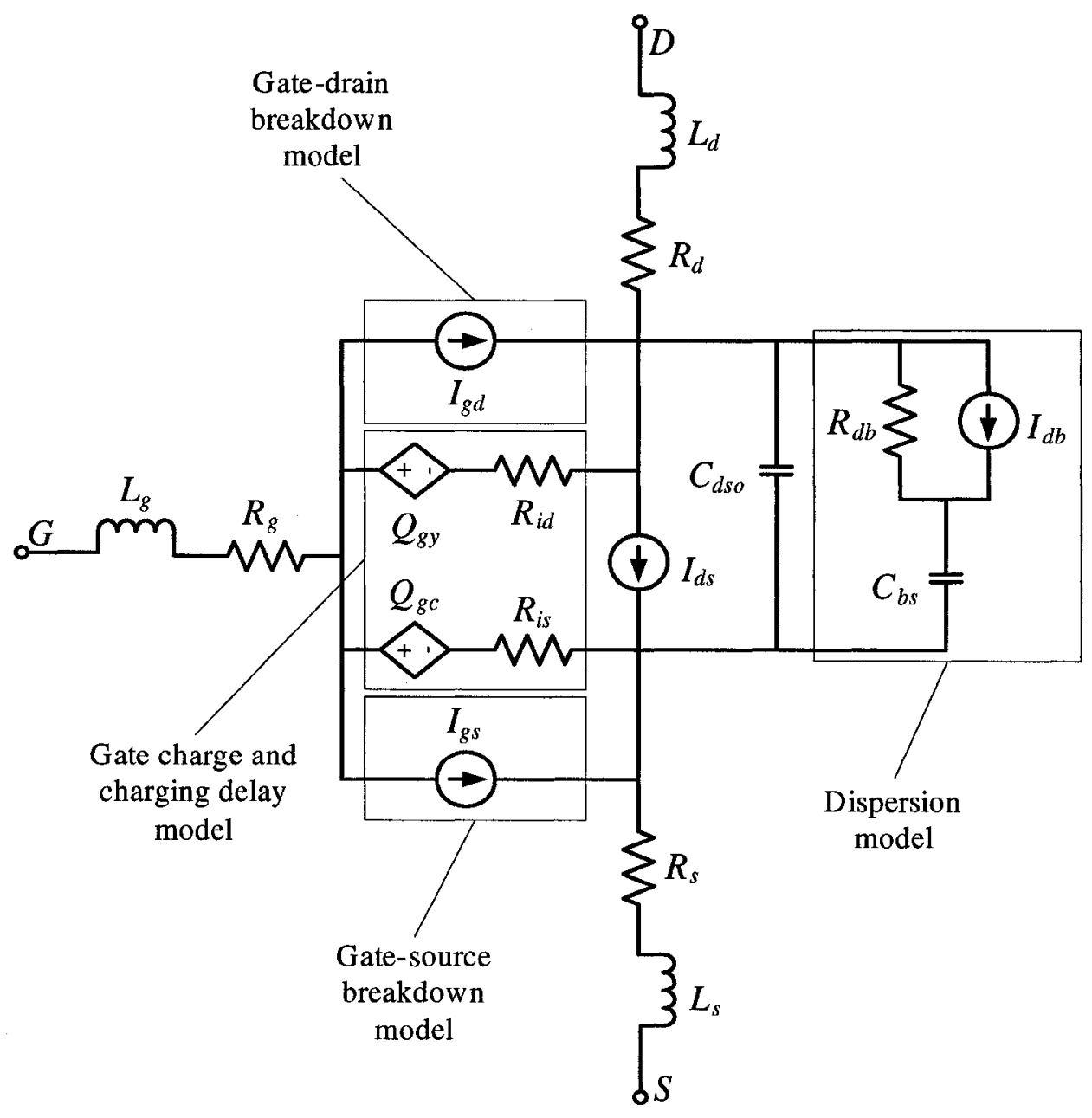

Figure 4.2: Agilent EEsof HEMT nonlinear model schematic. 


\subsubsection{Drain-Source Current Model}

The drain-source current $\left(I_{d s}\right)$ model, adapted from [45], fits analytic expressions to isothermal current-voltage measurements. Depending on the DC transconductance $\left(g_{m}\right)$ and gate-source voltage $\left(V_{g s}\right)$ profiles, $I_{d s}$ and output conductance $\left(g_{d s}\right)$ are evaluated in four distinct regions: below gate pinch-off (or gate threshold) voltage $\left(V_{p}\right)$, between $V_{p}$ and peak $g_{m}$, the region where $g_{m}$ is constant, and where $g_{m}$ compresses.

\subsubsection{Dispersion Model}

In his article [58], Ladbrooke defines dispersion as a "term used to indicate that the dynamic (or RF) characteristics of a device are different from the static (or DC) characteristics". Dispersion has two main sources: one is trapped charge, and the other is self-heating.

\section{Charge Trapping}

Charge accumulation at the surface or in the buffer layer of GaN HEMTs typically leads to reduced channel current and decreased output power from the device. These effects are modeled by the dispersion circuit proposed by Golio et al. [57] and shown in Figure 4.2.

The GaN HEMTs used in this work have been refined such that surface and buffer charge trapping effects are negligible, and therefore not included as part of the device model [59]. The interested reader is referred to [60-63] for more information on buffer layer effects and surface charge trapping phenomena. 


\section{Self-Heating}

Although a consequence of DC power dissipation in the device, self-heating occurs in a complicated and often misunderstood manner at RF. Under static conditions, the applied voltage and the resulting current change slowly enough to allow the lattice temperature of the device to reach steady state at each bias point. Self-heating is a manifestation of the DC power dissipated through a HEMT which, for the most part, is determined by the DC bias conditions. As the lattice temperature increases at higher voltages, the selfheating mechanism increases phonon scattering which, in turn, reduces carrier mobility and saturation velocity [64]. However, once a large-signal RF stimulus is superimposed onto the DC bias, the lattice temperature cannot adapt (the thermal time constant of the lattice is long compared to the rate of change of the RF signal) and so remains fixed by the bias point. The effects of self-heating, such as reduced transconductance and negative output conductance, are well-known and discussed in more detail in [65] and [66].

In this case, the EEHEMT model is fit to pulsed current-voltage measurements taken at deep Class $\mathrm{AB}$ biasing conditions. Pulsed measurements performed at a static quiescent point not only captures the RF behaviour of the GaN HEMT operated as a power amplifier, but also provides steady-state thermal conditions for the measurements. Indeed, the EEHEMT model comes complete with a thermal model that predicts the negative conductance effects from self-heating under static measurements, but is appropriately excluded as per the latter discussion [56]. As a precautionary measure however, large aluminum heat-sinks are used on all designs to mitigate inevitable thermal effects resulting from the RF cycle. 


\subsubsection{Gate and Output Charge Models}

The gate-charge model, shown in Figure 4.2, fits bias-dependent capacitance profiles extracted from measured y-parameters [53]. The depletion and channel regions are represented by two charge sources, $Q_{g c}$ and $Q_{g y}$, that model gate capacitance, and two corresponding resistances, $R_{i s}$ and $R_{i d}$, that model charging delay between the channel and depletion region.

The output capacitance, $C_{d s o}$, is a constant parameter, which gives the drain-source charge as a function of $V_{d s}$.

\subsubsection{Gate Forward Conduction and Breakdown Model}

Gate forward conduction is modeled by the standard diode equation, which is a function of $V_{g s}$ and diode ideality factor, $\eta[67]$. The GaN Schottky diode exhibits some peculiar characteristics that appear during model generation, suggesting that the well-known diode equation is not appropriate for this device.

The limited availability of die for this work prevents the development of the gateto-source and gate-to-drain breakdown models. Measuring such device characteristics typically leads to catastrophic device failure. The manufacturer has indicated a gate-todrain breakdown voltage level well above the peak voltage required for the Class E PA in this work. 


\subsection{Transistor Characterization}

The discussion on self-heating in Section 4.2.2 implies that the RF signal sees a unique set of dynamic current-voltage characteristics for each static (or DC) bias point. In theory, Class E PA operation requires a pinched-off gate bias level. The $V_{d s}$ for the Class E PA in Chapter 5 is $20 \mathrm{~V}$; at this bias, the gate pinch-off voltage is approximately $-3 \mathrm{~V}$. The GaN HEMT used in this thesis is designed to be operated at a $28 \mathrm{~V}$ drain bias in deep Class AB mode. For this reason, the manufacturer-supplied dynamic current-voltage data is generated at a $V_{d s}$ of $28 \mathrm{~V}$ and a $V_{g s}$ of $-2.6 \mathrm{~V}$, which is sufficient for this application even though the bias deviates from Class E conditions.

A pulsed current-voltage measurement setup is used to extract all dynamic currentvoltage data. The voltage pulse-width, during which time the current is measured, is set short enough (200 ns) to ensure that the true RF behaviour is captured [58]. A Hewlett-Packard 8510 Vector Network Analyzer, also with bias-tees, is used for on-wafer bias-dependent s-parameter measurements $[68]^{1}$.

As a starting point, extrinsic parameters are determined. Next, the model is fit to $I_{d s}-V_{d s}$ and DC $g_{m}$ profiles, as well as to the Schottky gate forward bias measurement. Input and output capacitances and model-generated, bias-dependent s-parameters are fit to measurements as a final step in the device modeling process. This process is iterative with many trade-offs in modeling accuracy, so some model parameters are adjusted away from extracted values in order to obtain the best possible fit to measured data. Optimized

\footnotetext{
${ }^{1}$ All device measurements in this section were conducted by Nitronex Corporation in order to assist with model development carried out by the author.
} 
model parameters are summarized in tables throughout this section wherever appropriate.

\subsubsection{Extrinsic Parameters}

The extrinsic model parameters which, in Figure 4.3, consist of $R_{g}, R_{s}, R_{d}, L_{g}$, $L_{s}$, and $L_{d}$, are determined using small-signal techniques presented by Dambrine et al. in [69].

The region underneath the gate of the device can be modeled as a distributed circuit

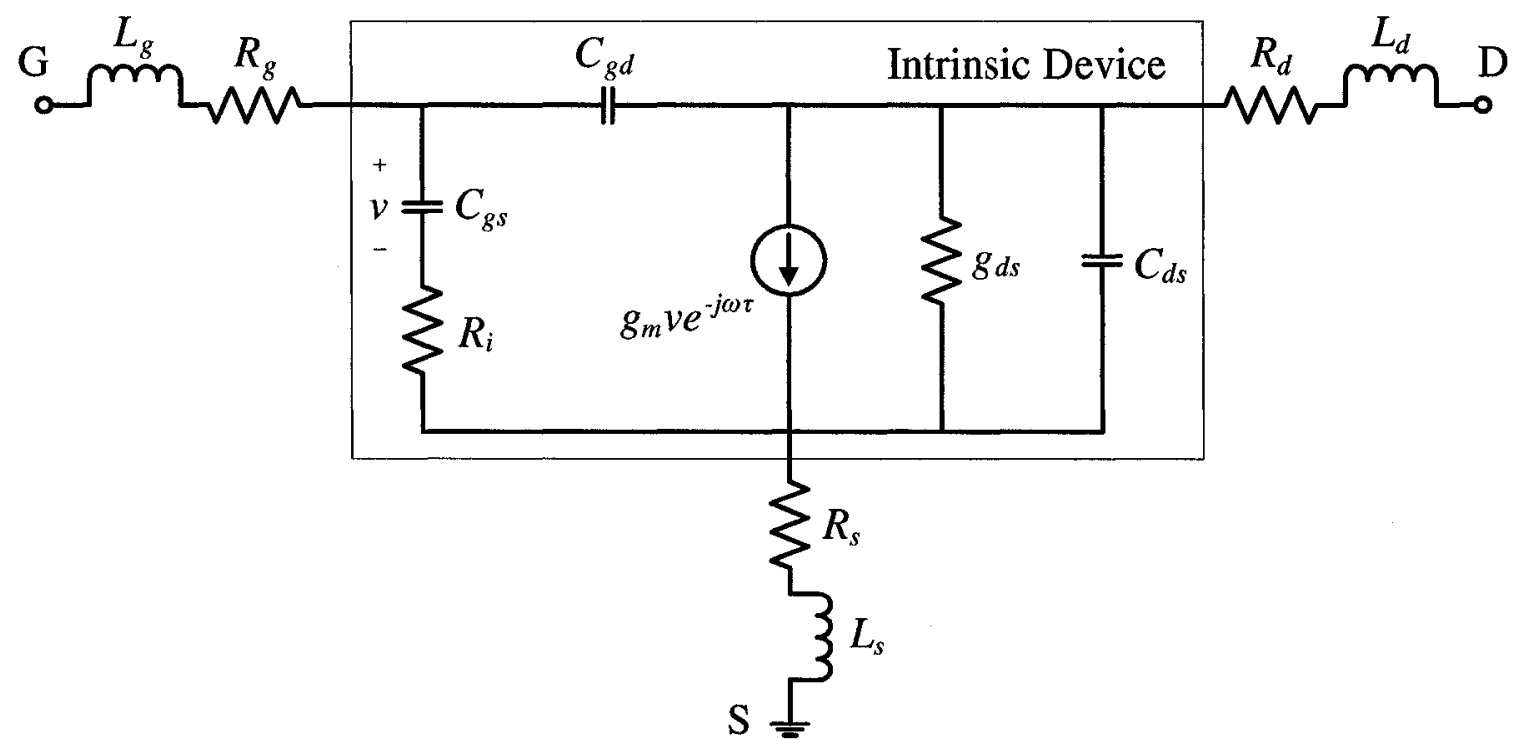

Figure 4.3: HEMT small signal model.

Gate

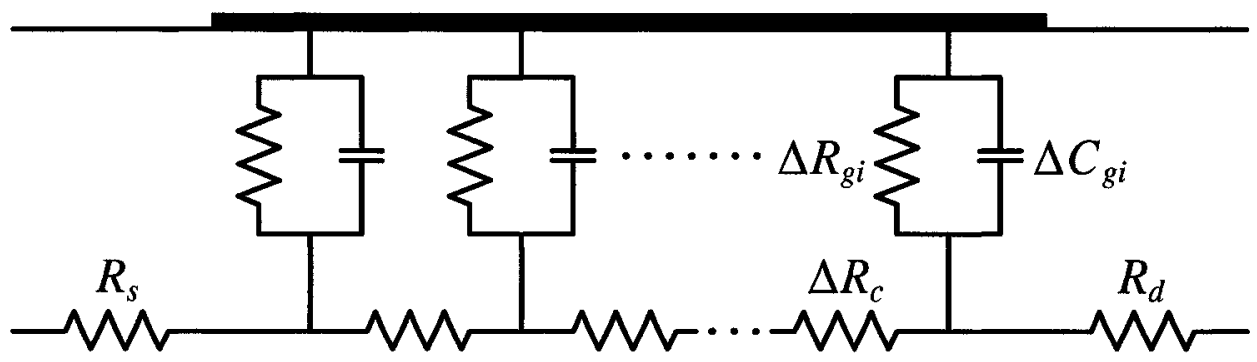

Figure 4.4: Distributed $R C$ transmission line representation of the region underneath the gate when $V_{d s}=0 \mathrm{~V}$.

as shown in Figure 4.4 under the condition that $V_{d s}=0 \mathrm{~V}$. Under this condition, the 
simplified model yields

$$
\begin{gathered}
Z_{11}=R_{s}+R_{g}+\frac{R_{c}}{3}+\frac{\eta k T}{q I_{g}}+j \omega\left(L_{s}+L_{g}\right) \\
Z_{12}=Z_{21}=R_{s}+\frac{R_{c}}{2}+j \omega L_{s} \\
Z_{22}=R_{s}+R_{d}+R_{c}+j \omega\left(L_{s}+L_{d}\right)
\end{gathered}
$$

where $R_{c}$ is the channel resistance, $I_{g}$ is the DC gate current, $\eta$ is the diode ideality factor, $k$ is Boltzmann's constant, and $T$ is the temperature. When the gate is heavily forward biased at a frequency lower than $5 \mathrm{GHz}$, the distributed resistance, $\Delta R_{g i}$, underneath the gate contact forms a short circuit across the distributed capacitance, $\Delta C_{g i}$. Figure 4.5 shows how $\Re\left\{Z_{11}\right\}$ varies with $\frac{1}{I_{g}}$ at $1 \mathrm{GHz}$. Linear extrapolation of $\Re\left\{Z_{11}\right\}$ to the

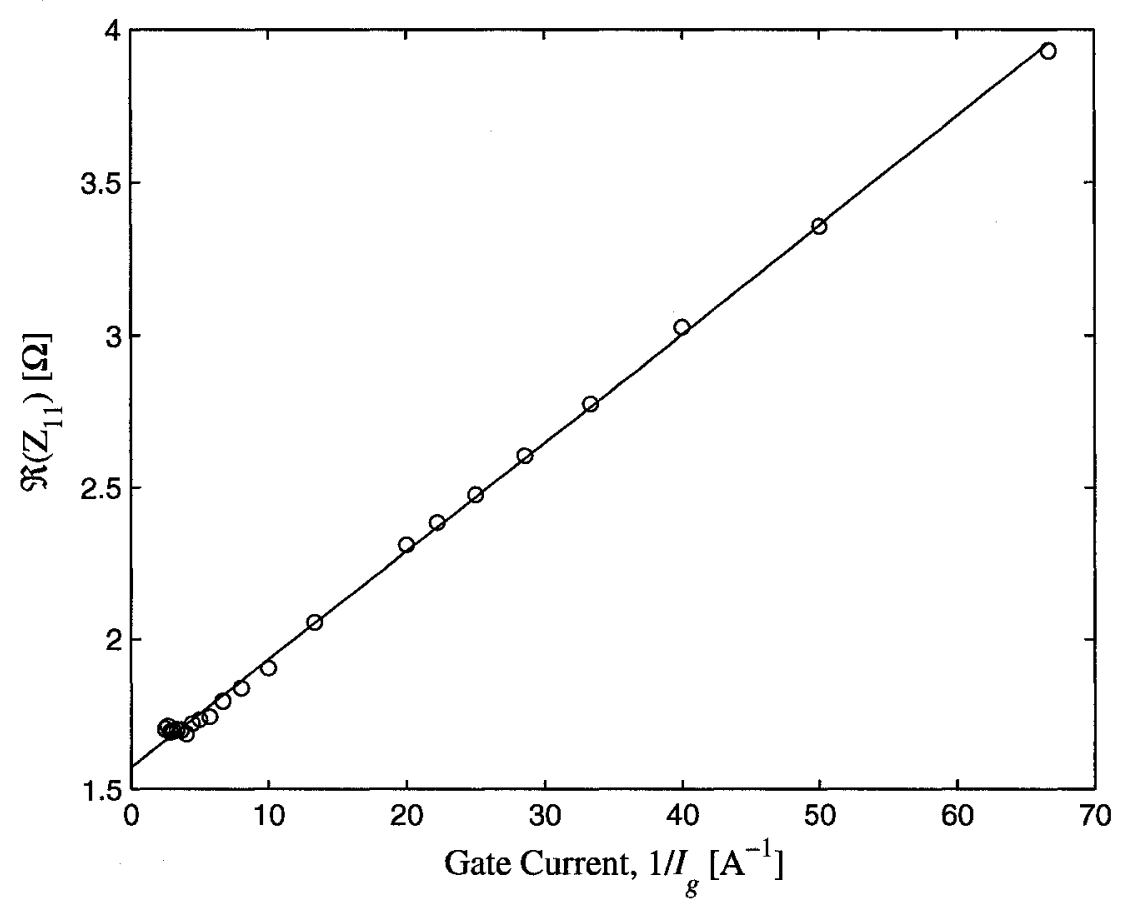

Figure 4.5: The real part of $Z_{11}$ plotted as a function of $\frac{1}{I_{g}}$ at $1 \mathrm{GHz}$ under the condition that $V_{d s}=0 \mathrm{~V}$. 
$\frac{1}{I_{g}}=0$ axis equates the fourth term in Equation 4.3.1 to zero, giving the expression in Equation 4.3.4.

$$
\left.\Re\left\{Z_{11}\right\}\right|_{I_{g} \rightarrow \infty}=R_{s}+R_{g}+\frac{R_{c}}{3}=1.551 \Omega
$$

The real parts of Equations 4.3.1 - 4.3.3 and Equation 4.3.4 represent five unknowns in four equations. In the absence of a fifth equations, the extrinsic elements in the symbolic forms of Equations 4.3.1 - 4.3.3 are optimized to fit both measured z-parameters and the condition in Equation 4.3.4. The schematic in Figure 4.6(a) models Equation 4.3.1 and the schematic in Figure 4.6(b) models Equations 4.3.2 and 4.3.3.

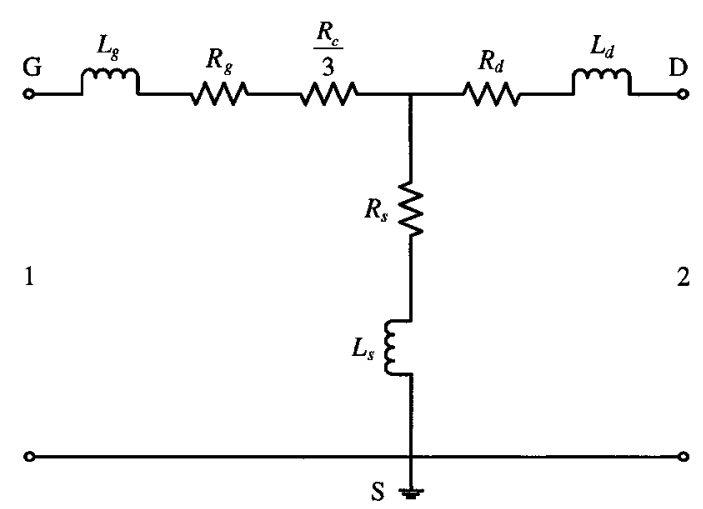

(a)

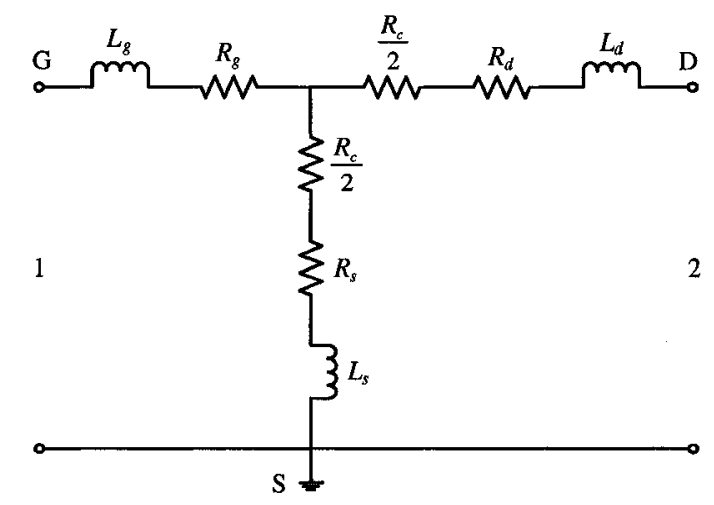

(b)

Figure 4.6: Schematic representations of (a) Equation 4.3.1 and (b) Equations 4.3 .2 and 4.3 .3

Initial estimates of the extrinsic inductances are obtained from the imaginary parts of Equations 4.3.1- 4.3.3. Figure 4.7 shows $\Im\left\{Z_{11}\right\}, \Im\left\{Z_{12}\right\}$, and $\Im\left\{Z_{22}\right\}$ plotted versus frequency up to $5 \mathrm{GHz}$. As expected, the linear progression of the imaginary parts of the z-parameters indicate fixed extrinsic inductances with respect to frequency. Using Equations 4.3.1 - 4.3.3, it is deduced from Figure 4.7 that $L_{s} \approx 1.52 \mathrm{pH}, L_{g} \approx 67.84$ $\mathrm{pH}$, and $L_{d} \approx 98 \mathrm{pH}$. This information is used in an ADS optimization routine where the 


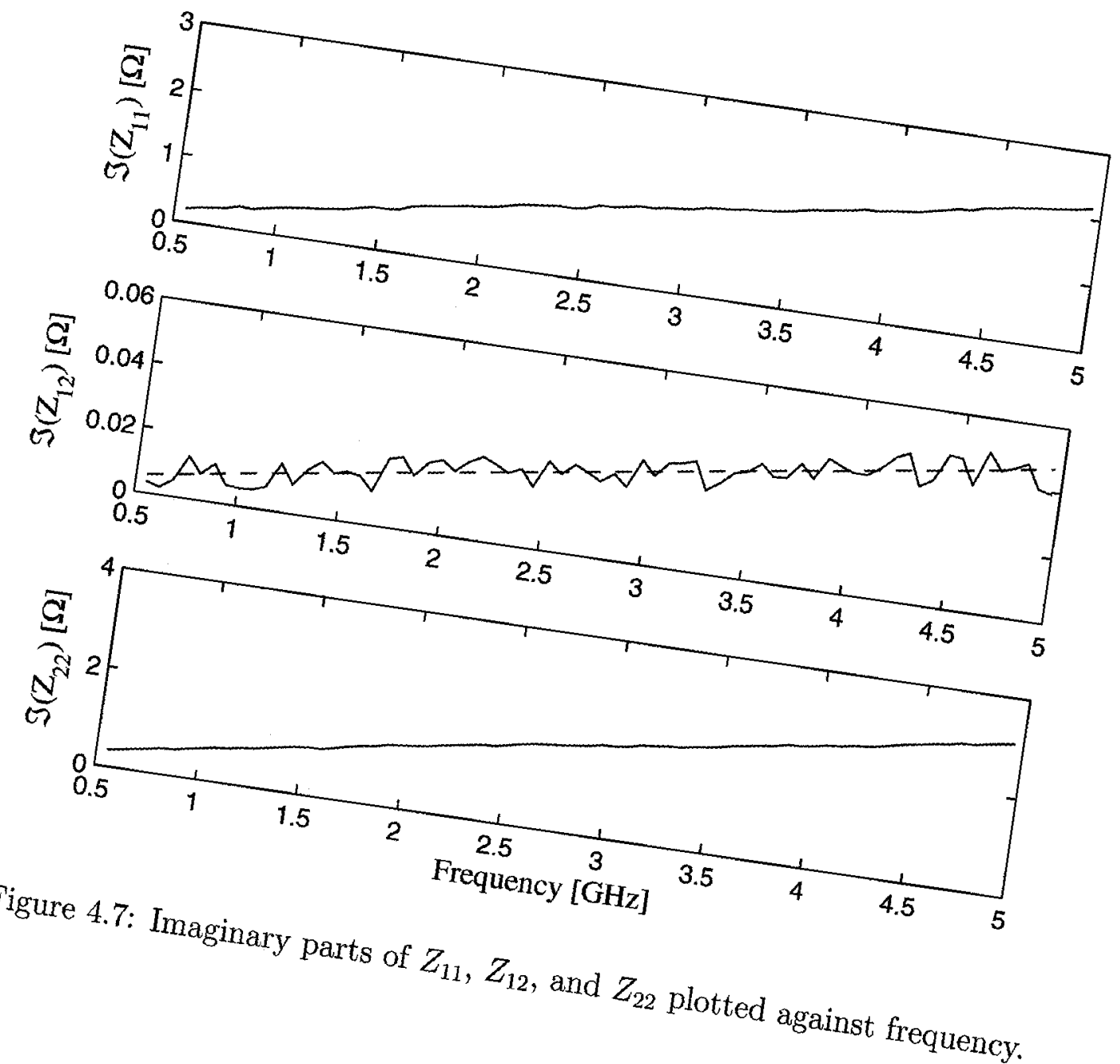

Reproduced with permission of the copyright owner. Further reproduction prohibited without permission. 
circuit elements are set as optimization variables and Equation 4.3.4 and the forward-gate z-parameters measured at an $I_{g}$ of $500 \mathrm{~mA}$ are used as simulation targets.

Owing to the bias independence of the extrinsic elements, the values obtained here are further constrained to fit small-signal measurements performed on the device at any bias. In this process, the intrinsic device parameters in Figure 4.3 are extracted as functions of applied bias while monitoring any variation of the extrinsic elements. The extrinsic elements remain constant with respect to bias, as shown in Figure 4.8 and summarized in Table 4.2 .
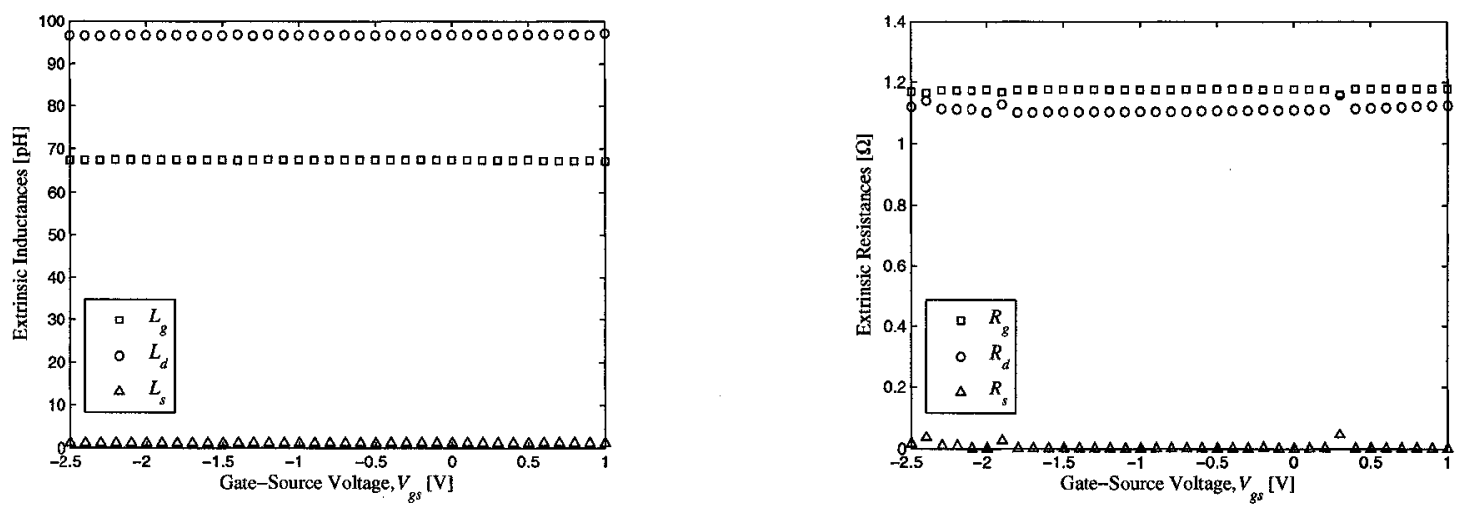

Figure 4.8: Extrinsic resistances and inductances extracted from small signal model optimization with respect to $V_{g s}$ at $V_{d s}=20 \mathrm{~V}$.

\begin{tabular}{|c|c|c|c|c|c|}
\hline$L_{g}$ & $L_{d}$ & $L_{s}$ & $R_{g}$ & $R_{d}$ & $R_{s}$ \\
\hline \hline $67.3 \mathrm{pH}$ & $96.5 \mathrm{pH}$ & $1.125 \mathrm{pH}$ & $1.176 \Omega$ & $1.1 \Omega$ & $0 \Omega$ \\
\hline
\end{tabular}

Table 4.2: Optimized extrinsic parameter values.

\subsubsection{Drain-Source Current and Transconductance}

The current-voltage characteristics are determined using $g_{d s}$, DC $g_{m}$, and the behaviour of $I_{d s}$ as a function of $V_{g s}$. Indeed, these attributes also depend upon the value of $V_{d s}$ 
at which they are analyzed, but the $V_{d s}$ dependency can be removed from many of the equations to simplify parameter extraction, and then later re-integrated as the model is tuned over bias. This feature is an integral part of the EEHEMT model via the model parameter $V_{d s o}$; in this case $V_{d s o}$ is set to $20 \mathrm{~V}$, which is the operating bias for the Class E PA in Chapter 5.

The only measurement in which the $V_{d s}$ dependency remains is in the $I_{d s}$ vs. $V_{d s}$ measurements. So, as a starting point, output conductance, $g_{d s}$, and saturation voltage, $V_{s a t}$, are determined from the $I_{d s}$ vs. $V_{d s}$ measurements in Figure 4.9. Taking the slope

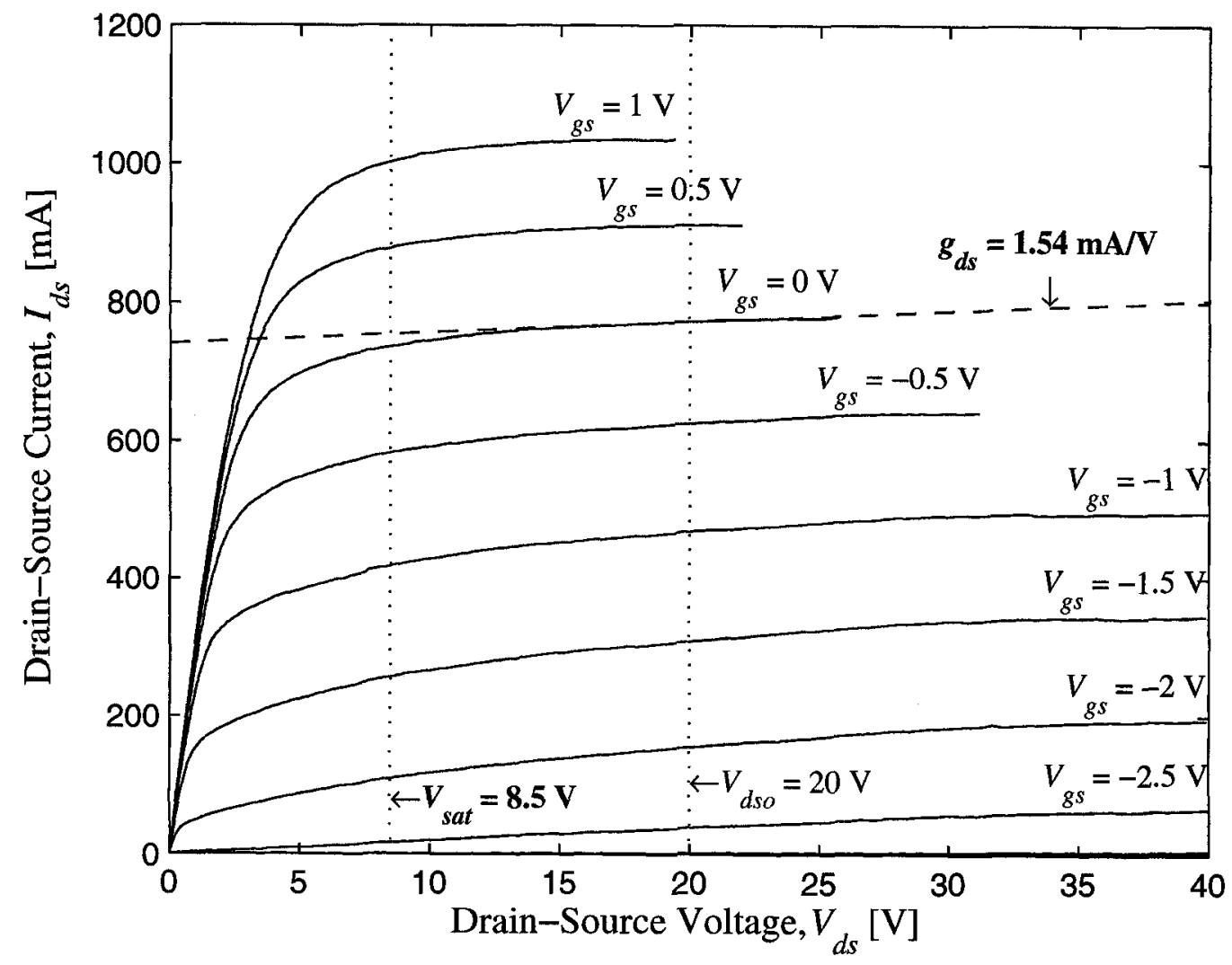

Figure 4.9: Pulsed current-voltage characteristics of the $2 \mathrm{~mm}$ GaN HEMT.

of the $V_{g s}=0 \mathrm{~V}$ curve at $V_{d s}=V_{d s o}$ gives a $g_{d s}$ of $1.54 \mathrm{~mA} / \mathrm{V}$. The voltage at which $I_{d s}$ 
saturates is estimated to be at $8.5 \mathrm{~V}$ for the same $V_{g s}$. The nonlinear equations contain additional parameters that model $V_{d s}$ dependent gate pinch-off (or short-channel effects) and drain current in the sub-threshold region. Although these properties are important, their relevance is only secondary. The model parameters that control these features are appropriately used as fitting parameters. Table 4.3 shows values for the fitting parameters as well as final optimized model parameters for this portion of the model.

\begin{tabular}{|c|c|c|}
\hline Parameter & Description & Value \\
\hline \hline$V_{d s o}$ & Typical operating $V_{d s}$ in saturation & $20 \mathrm{~V}$ \\
\hline$V_{s a t}$ & $V_{d s}$ at which $I_{d s}$ saturates & $8.5 \mathrm{~V}$ \\
\hline$g_{d s}($ Kapa $)$ & Output conductance in saturation & $0.01 \mathrm{~mA} / \mathrm{V}$ \\
\hline$G a m m a$ & $V_{d s}$ dependent pinch-off tuning parameter & 0.0025 \\
\hline$V_{c h}$ & $V_{g s}$ where effect of Gamma is removed & $2 \mathrm{~V}$ \\
\hline$V_{t s o}$ & Subthreshold onset voltage & $-19 \mathrm{~V}$ \\
\hline
\end{tabular}

Table 4.3: Summary of optimized model parameters obtained from current-voltage analysis. Parameters appearing in parentheses are the proper names used in the model's documentation where different variables are used in this document.

Transconductance is analyzed next; Figure 4.10 shows the DC $g_{m}$ vs. $V_{g s}$ response at $V_{d s}=V_{d s o}$. Using the details gathered from this plot, the model equations evaluate the $g_{m}$ profile in the four distinct regions mentioned in Section 4.2.1. The pertinent information is gathered by inspection: $V_{p}$ is $-3 \mathrm{~V}$; peak $g_{m}\left(g_{m m a x}\right)$ and the gate-source voltage at which the peak occurs $\left(V_{g o}\right)$ are $320 \mathrm{mS}$ and $-1.6 \mathrm{~V}$, respectively; and finally, the gate-source voltage at which $g_{m}$ begins to compress $\left(V_{c o}\right)$ and the slope of the compression $\left(\Delta g_{m}\right)$ are $-1 \mathrm{~V}$ and $30 \mathrm{mS} / \mathrm{V}$, respectively ${ }^{2}$. The model provisions abrupt $g_{m}$ compression and a "tail-off" feature where $g_{m}$ eventually goes to zero. This behaviour is not evident

\footnotetext{
${ }^{2}$ Proper variable definition of the slope of $g_{m}$ compression is $\frac{\Delta g_{m}}{\Delta V_{g s}} . \Delta g_{m}$ is a shortened form of the variable name used in the model's documentation.
} 


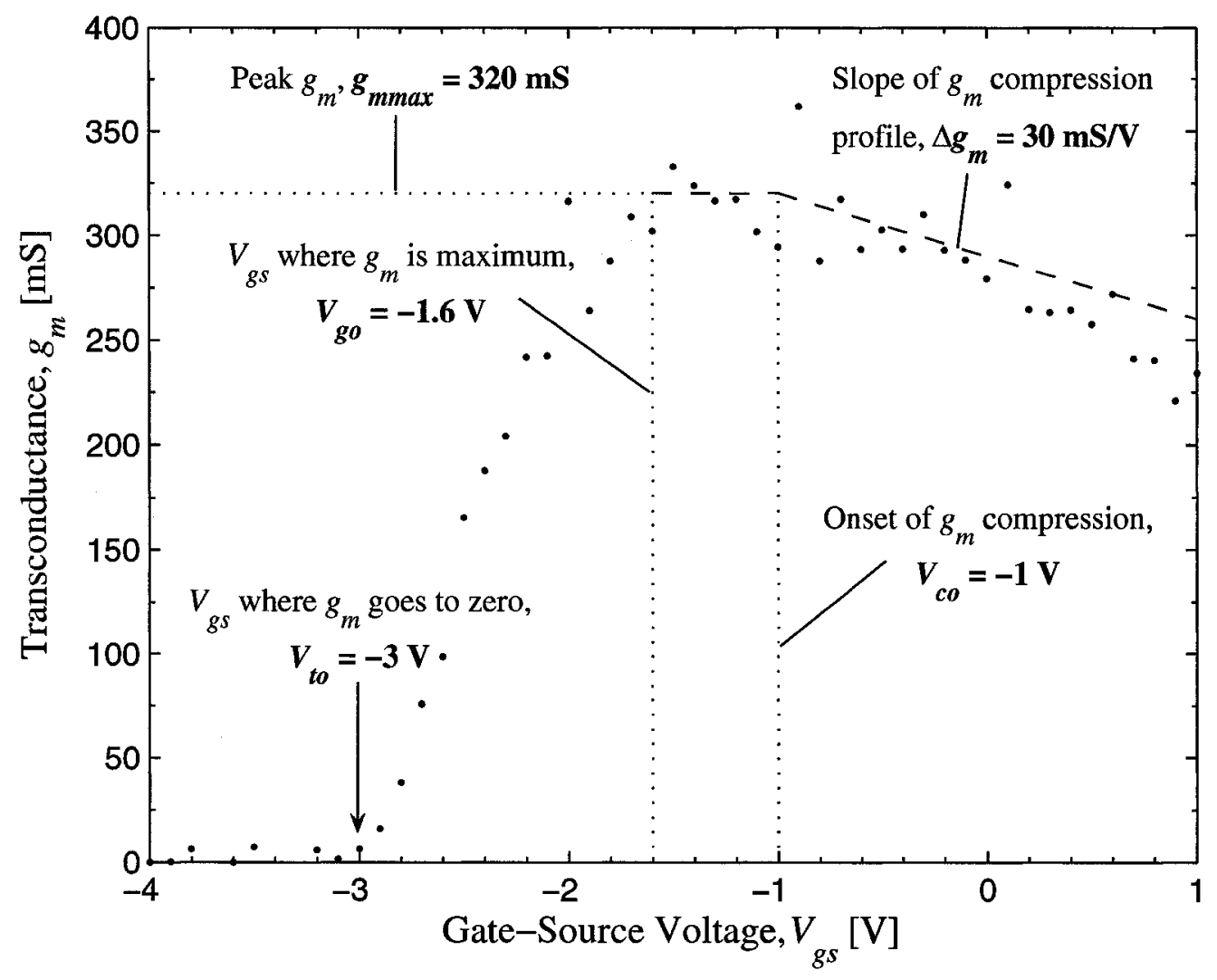

Figure 4.10: Pulsed $g_{m}$ profile at $V_{d s}=V_{d s o}=20 \mathrm{~V}$. DC $g_{m}$ is characterized below $V_{p}$, between $V_{p}$ and $g_{\text {mmax }}$, between $V_{g o}$ and $V_{c o}$, and beyond $V_{c o}$. 
from the plot in Figure 4.10, and so those additional model parameters are adjusted as needed during optimization. Table 4.4 summarizes the optimized model parameters for this segment of model extraction.

\begin{tabular}{|c|c|c|}
\hline Parameter & Description & Value \\
\hline \hline$V_{p}\left(V_{t o}\right)$ & Gate pinch-off voltage at $V_{d s}=V_{d s o}$ & $-3 \mathrm{~V}$ \\
\hline$g_{m m a x}$ & Peak DC $g_{m}$ & $330 \mathrm{mS}$ \\
\hline$V_{g o}$ & $V_{g s}$ where $g_{m m a x}$ occurs & $-1.6 \mathrm{~V}$ \\
\hline$V_{c o}$ & $V_{g s}$ where $g_{m}$ compression begins & $-1 \mathrm{~V}$ \\
\hline$\Delta g_{m}(D e l t g m)$ & Slope of $g_{m}$ compression $($ absolute value) & $30 \mathrm{mS} / \mathrm{V}$ \\
\hline$V_{b c}$ & Transition voltage between $V_{c o}$ and $g_{m}$ tail-off & $5 \mathrm{~V}$ \\
\hline$V_{b a}$ & $V_{g s}$ over which $g_{m}$ tails-off & $1 \mathrm{~V}$ \\
\hline$\mu(M u)$ & $V_{d s}$ dependent $V_{c o}$ correction factor & 0 \\
\hline$\alpha(A l p h a)$ & Controls abruptness of $g_{m}$ compression & $8.22(10)^{-7}$ \\
\hline
\end{tabular}

Table 4.4: Summary of optimized DC $g_{m}$ parameters. Parameters appearing in parentheses are the proper names used in the model's documentation where different variables are used in this document.

\subsubsection{Gate Forward Conduction}

The Schottky diode at the metal-AlGaN interface is modeled by Equation 4.3.5 shown below, where $I_{s}$ is the reverse saturation current and $q$ is the electronic charge.

$$
I_{g s}=I_{s}\left(e^{\frac{q V_{g s}}{\eta k T}}-1\right)
$$

Plotting $\ln \left(I_{g s}\right)$ against $V_{g s}$ as shown in Figure 4.11 and extrapolating the straight line portion to the $I_{g s}$ axis gives $I_{s}$ at $3(10)^{-12}$ A. The diode ideality factor, $\eta$, is determined by equating the slope of the same line to $\frac{q}{\eta k T}$ from Equation 4.3.5 [70]. The derivation gives $\eta=1.285$. Table 4.5 shows the optimized values for $\eta$ and $I_{s}$. Typical values for $\eta$ are between 1 and 2; values greater that 2 are non-physical. This phenomenon is 
responsible for some of the discrepancies in the device model as discussed in further detail in Section 4.4.

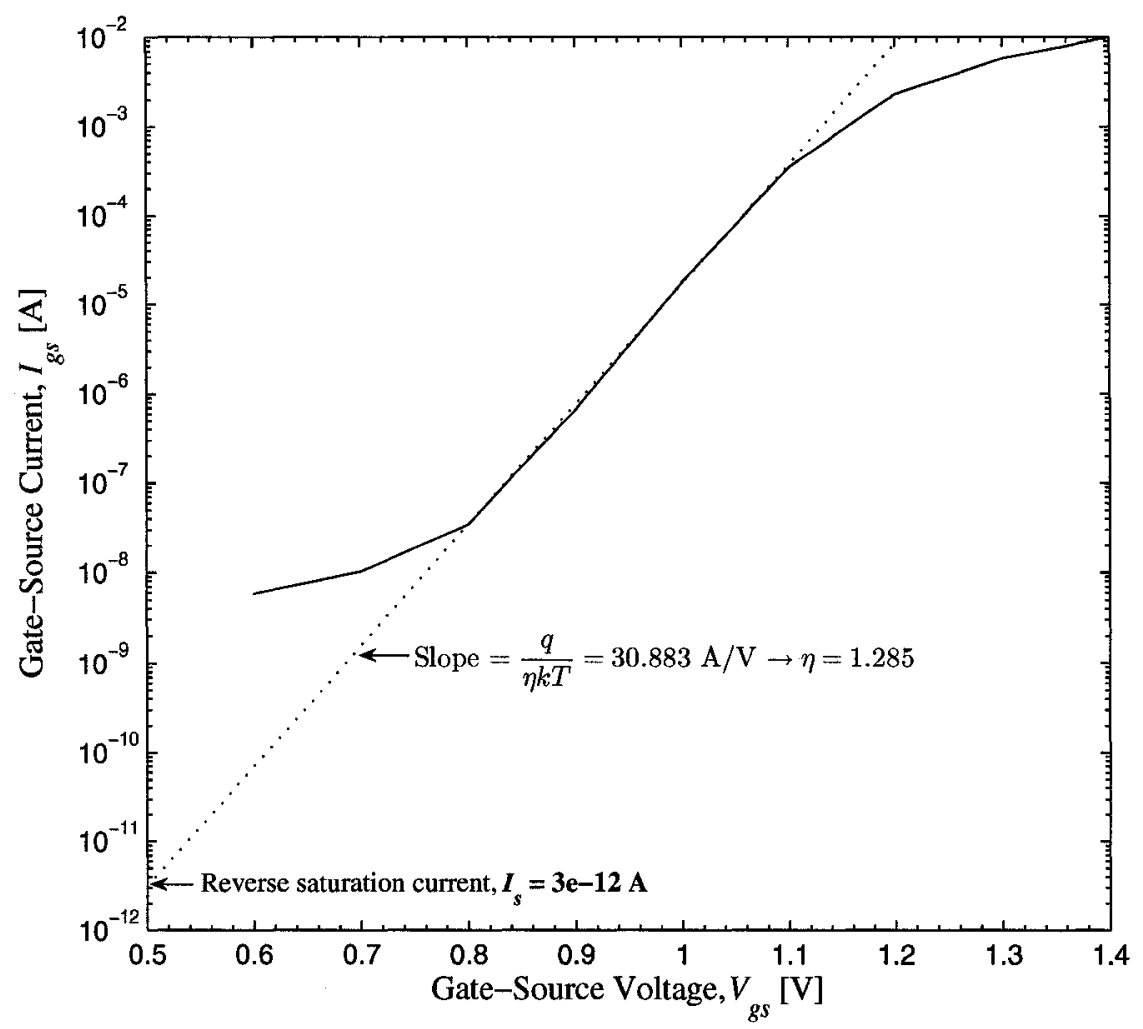

Figure 4.11: $I_{g s}$ vs. $V_{g s}$ measurement for Schottky diode characterization.

\begin{tabular}{|c|c|c|}
\hline Parameter & Description & Value \\
\hline \hline$I_{s}$ & Schottky diode reverse saturation current & $3(10)^{-12} \mathrm{~A}$ \\
\hline$\eta(N)$ & Schottky diode ideality factor & 2.05 \\
\hline
\end{tabular}

Table 4.5: Summary of optimized parameters for the Schottky diode model. Parameters appearing in parentheses are the proper names used in the model's documentation where different variables are used in this document.

\subsubsection{Gate and Output Capacitance}

Model parameters representing gate and output charge depend primarily on small-signal characteristics of the device. This part of the model represents the largest portion of 
model optimization, as many of the model parameters are automatically adjusted (from initial estimates) to fit measured bias-dependent small-signal device behaviour.

The nonlinear model schematic in Figure 4.2 shows the gate charge being split between two charge sources, $Q_{g c}$ and $Q_{g y}$. These charge sources correspond to the input capacitances $C_{11}$ and $C_{12}$, where port one is taken at the gate and port two at the drain. The capacitance parameter extraction procedure starts with s-parameter measurements taken at $V_{d s}=V_{d s o}$, similar to the manner in which the $g_{m}$ model is generated in Section 4.3.2. The characteristic $C_{11}$ curve in Figure 4.12, from measured s-parameters, reveals a maximum input capacitance $\left(C_{110}\right)$ of $3.401 \mathrm{pF}$. The downward trend of $C_{11}$ beyond a $V_{g s}$ of $-2 \mathrm{~V}$ is an artifact of the small-signal measurement method used to extract the input capacitance profile. It does not suggest that the transistor is forward biased. The model assumes a constant input capacitance below gate pinch-off, so the minimum input capacitance $\left(C_{11 t h}\right)$ is approximated at $1.36 \mathrm{pF}$ even though it is clear from the plot that the absolute minimum falls below this value. The model uses the voltage difference between $C_{11 t h}$ and $C_{11 o}$, and the curve's inflection point (at $V_{\text {infl }}$ ) to complete the characterization; $\Delta V_{g s}$ and $V_{\text {infl }}$ are $1 \mathrm{~V}$ and $-2.6 \mathrm{~V}$, respectively. The linear to saturation region transition of the gate capacitance is reflected by the model parameter, $\Delta V_{d s}$, as a change in $V_{d s}$. Figure 4.13 shows $C_{11}$ plotted as a function of $V_{d s}$. The transition voltage is clear for $V_{g s}=1 \mathrm{~V}$, and so $\Delta V_{d s}$ is set to $6.5 \mathrm{~V}$ as an initial estimate

The model assumes a linear voltage dependency of the gate-drain charge in saturation. The resulting constant gate-drain capacitance, $C_{g d s a t}$, is estimated using bias-dependent small-signal model extraction using the schematic in Figure 4.3. Figure 4.14 shows how 


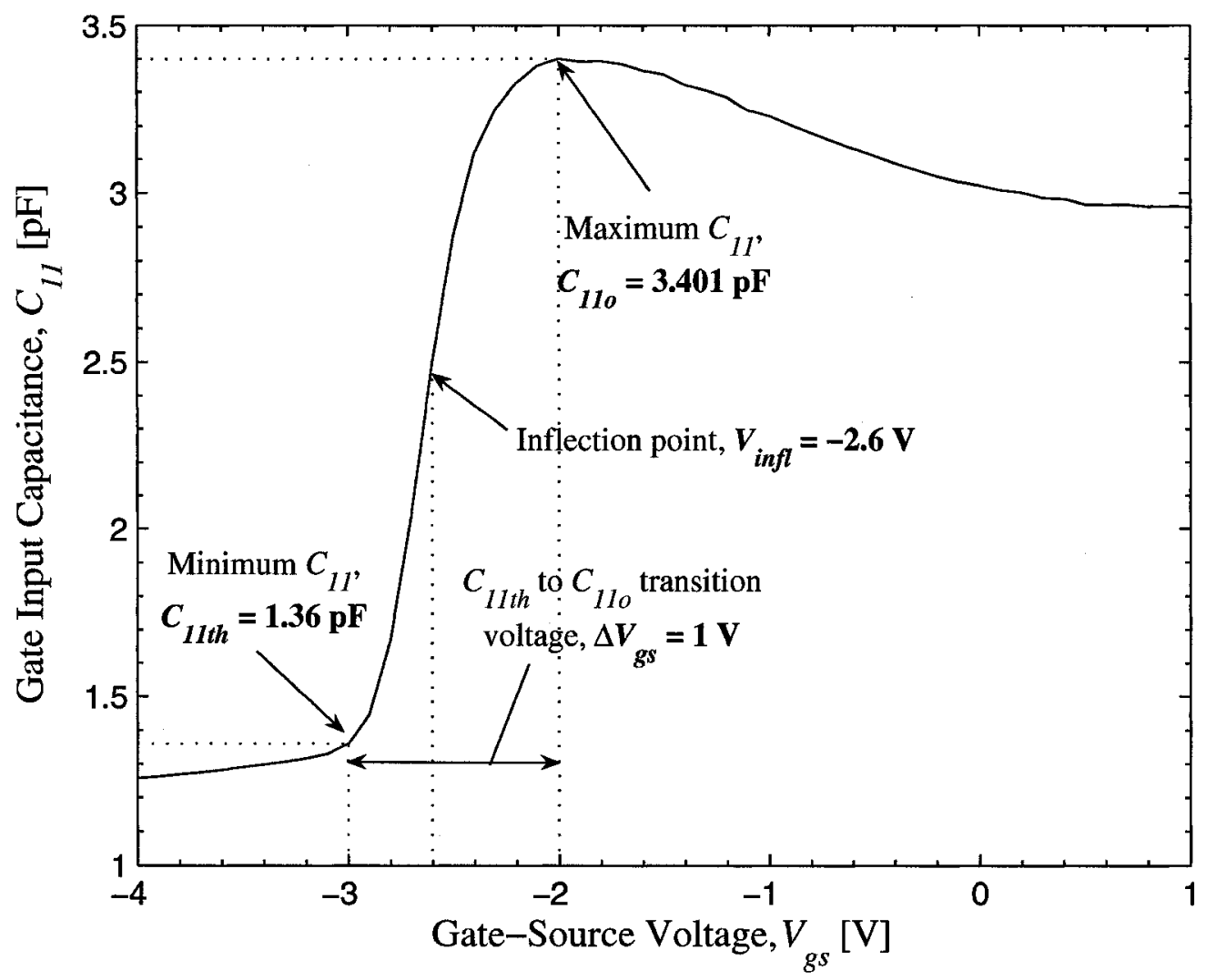

Figure 4.12: Input capacitance, $C_{11}$, plotted vs. $V_{g s}$ at $V_{d s}=V_{d s o}$ is used to characterize the nonlinear input capacitance. 


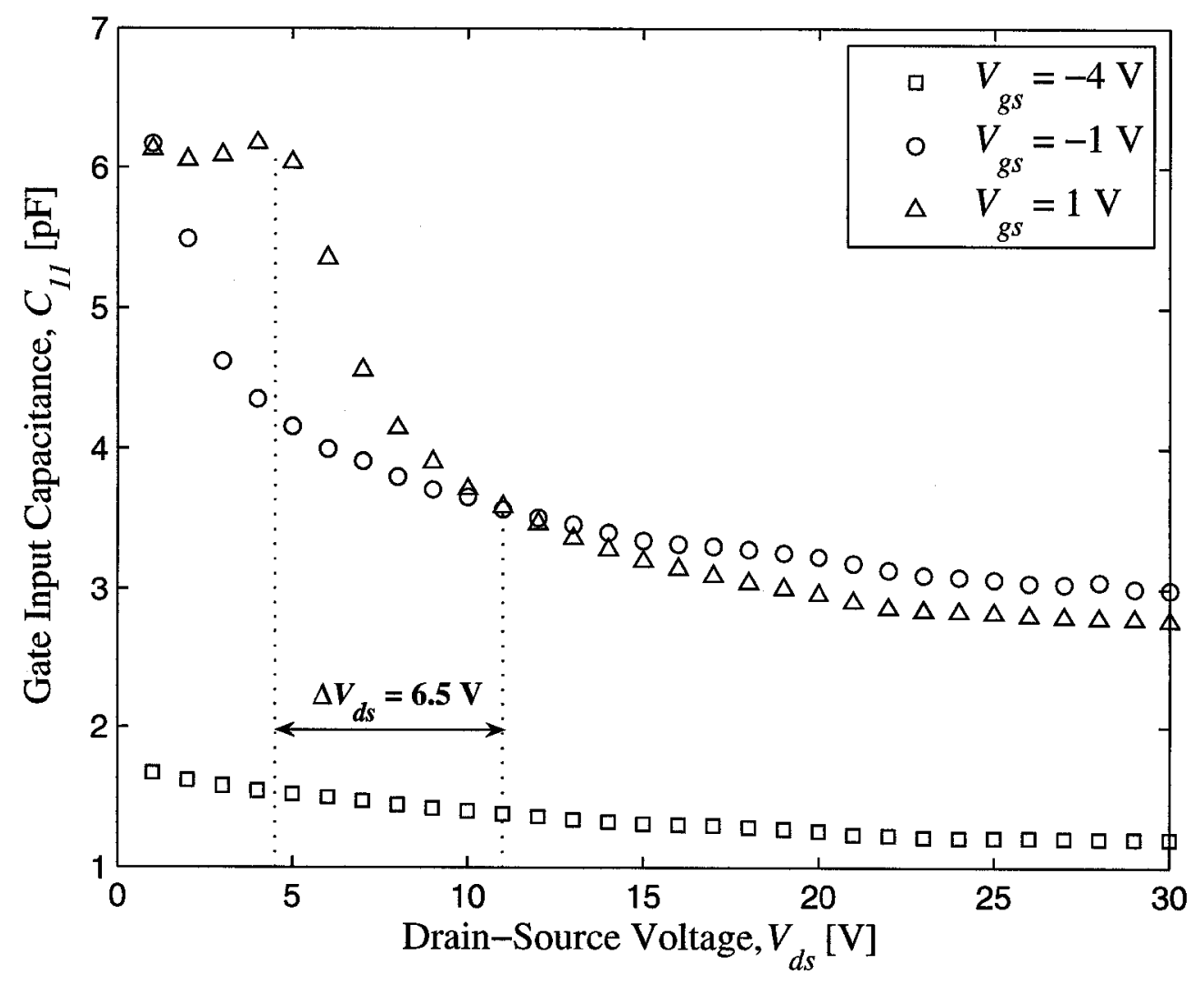

Figure 4.13: Input capacitance, $C_{11}$, plotted against $V_{d s}$, shows the range of $V_{d s}$ over which $C_{11}$ transitions into the saturation region. 
$C_{g d}$ varies with $V_{g s}$; from this plot, the model parameter, $C_{g d s a t}$, is estimated to be 0.32 $\mathrm{pF}$. The parameter, $\lambda$, described in the model's documentation as a " $C_{11}-V_{d s}$ slope parameter" is used as a correction factor in the tuning phase.

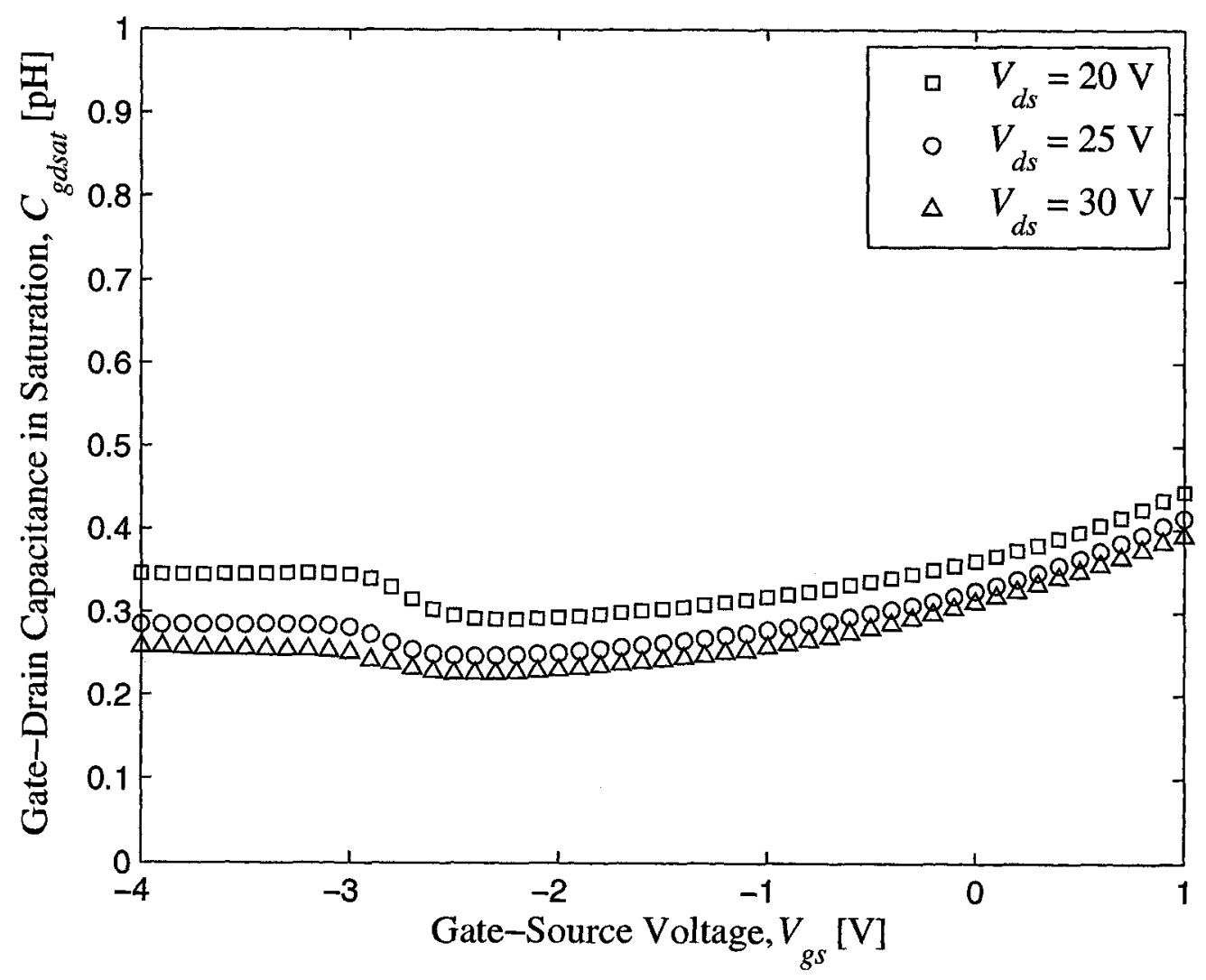

Figure 4.14: Gate-drain capacitance, $C_{g d}$, plotted vs. $V_{g s}$ for $V_{d s}=20 \mathrm{~V}, V_{d s}=25 \mathrm{~V}$, and $V_{d s}=30 \mathrm{~V} . C_{g d}$ is assumed constant by the model; the dependency of $C_{g d}$ on $V_{g s}$ is therefore ignored.

Finally, the output capacitance is easily obtained from Figure 4.15, which shows a roughly constant $C_{d s}$ as a function of $V_{d s}$. The output capacitance is typically a linear quantity; the model consequently predicts a linear evolution of drain-source charge with $V_{d s}$. The model parameter that defines drain-source capacitance, $C_{d s o}$, is set to an initial estimate of $0.27 \mathrm{pF}$. 


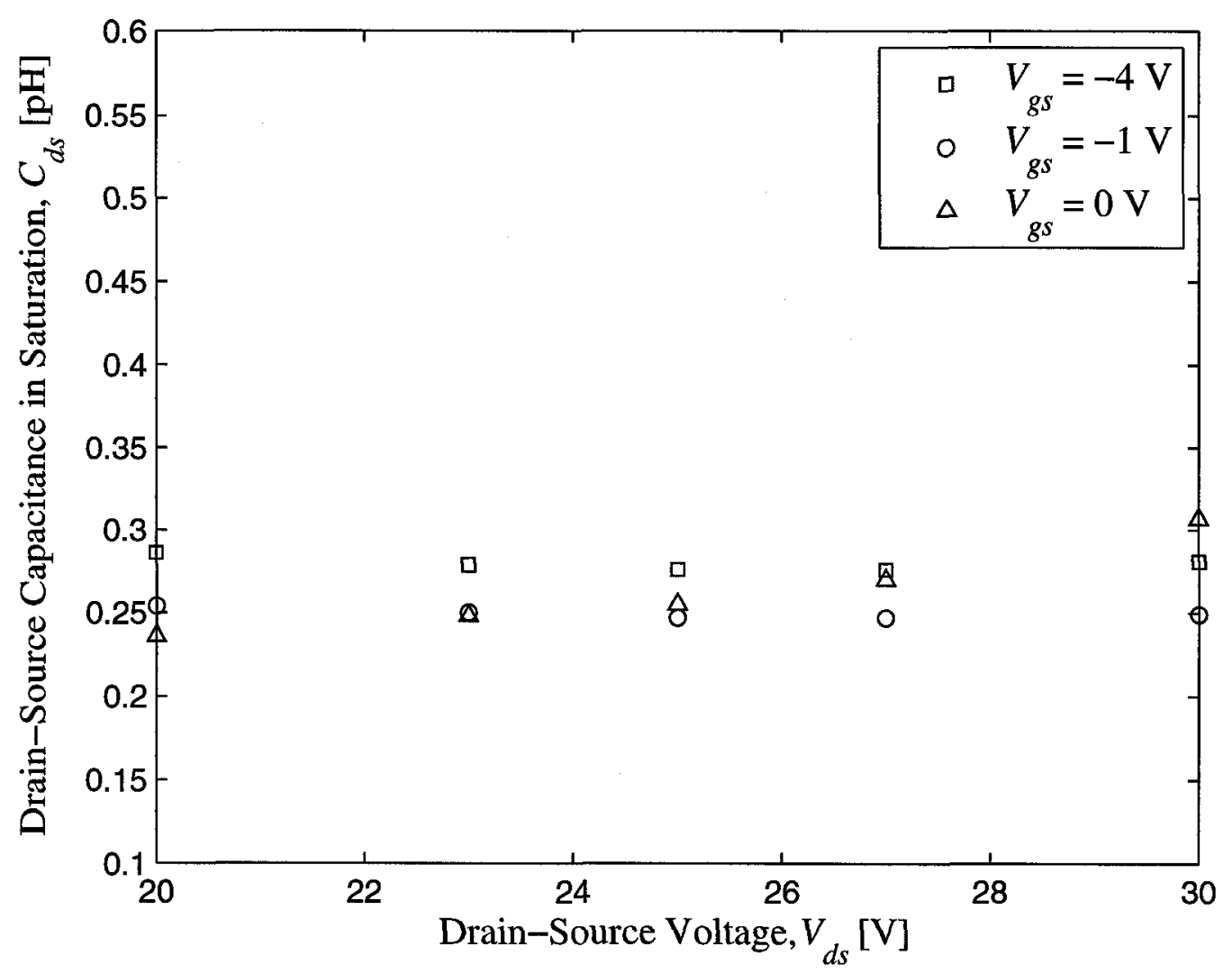

Figure 4.15: Output capacitance, $C_{d s}$, plotted vs. $V_{d s}$ for output capacitance extraction. $C_{d s}$ is constant with respect to $V_{d s}$, as assumed by the nonlinear model. 
The remaining charge parameters model the input transcapacitance in saturation $\left(C_{12 s a t}\right)$ and charging delay between the channel and depletion region, either in the form of channel resistances, $R_{i s}$ and $R_{i d}$, or using the drain-source current delay time constant, $\tau$. These parameters are tuned to provide optimal correlation between measured and modeled device behaviour in both the large- and small-signal regimes.

Table 4.6 summarizes the optimized parameters that characterize the input and output charge model.

\begin{tabular}{|c|c|c|}
\hline Parameter & Description & Value \\
\hline \hline$C_{110}$ & Maximum input capacitance for $V_{d s}=V_{d s o}$ & $3.399 \mathrm{pF}$ \\
\hline$C_{11 t h}$ & Minimum input capacitance for $V_{d s}=V_{d s o}$ & $1.359 \mathrm{pF}$ \\
\hline$V_{\text {infl }}$ & $C_{11}$ vs. $V_{g s}$ inflection voltage & $-2.708 \mathrm{~V}$ \\
\hline$\Delta V_{g s}($ Deltgs $)$ & Transition voltage between $C_{11 t h}$ and $C_{110}$ & $0.931 \mathrm{~V}$ \\
\hline$\Delta V_{d s}($ Deltds $)$ & $\begin{array}{c}\text { Linear to saturation region transition } \\
\text { voltage in } C_{11} \text { vs. } V_{d s} \text { profile }\end{array}$ & $6.972 \mathrm{~V}$ \\
\hline$\lambda($ Lambda $)$ & $C_{11}$ vs. $V_{d s}$ slope parameter & 0.003 \\
\hline$C_{12 s a t}$ & Input transcapacitance in saturation & $0.284 \mathrm{pF}$ \\
\hline$C_{g d s a t}$ & $C_{g d}$ in saturation & $0.344 \mathrm{pF}$ \\
\hline$C_{d s}\left(C_{d s o}\right)$ & Drain-source output capacitance & $0.28 \mathrm{pF}$ \\
\hline$R_{i d}$ & Channel resistance at the drain & $8.22 \Omega$ \\
\hline$R_{i s}$ & Channel resistance at the source & $2.08 \Omega$ \\
\hline$\tau($ Tau $)$ & $I_{d s}$ delay time constant & $7.269 \mathrm{ps}$ \\
\hline
\end{tabular}

Table 4.6: Summary of optimized parameters that describe the gate and drain charge profiles and charging delay. Parameters appearing in parentheses are the proper names used in the model's documentation where different variable names are used in this document.

\subsection{Model Verification}

The complete optimized nonlinear model can be found in Appendix A. In general, the model accurately fits measured data over wide-ranging operating conditions:

- $I_{d s}$ is predicted from $0 \mathrm{~A}$ to $1 \mathrm{~A}$ with respect to $V_{d s}$ up to $20 \mathrm{~W}$ of DC power. 
- $I_{d s}$ is predicted from $0 \mathrm{~A}$ to $1 \mathrm{~A}$ with respect to $V_{g s}$ from $-4 \mathrm{~V}$ to $1 \mathrm{~V}$.

- DC $g_{m}$ is predicted for $V_{g s}$ from $-4 \mathrm{~V}$ to $1 \mathrm{~V}$ and $V_{d s}$ up to $40 \mathrm{~V}$.

- With the exception of near pinch-off and forward gate bias levels, the small-signal model predicts measured performance up to $20 \mathrm{GHz}$.

- Large-signal power characteristics are predicted for Class A biasing conditions.

Having completed model generation of the $2 \mathrm{~mm}$ GaN-on-Si HEMT, a simulated vs. measured data comparison is presented in this section, starting with $I_{d s}$ vs. $V_{d s}$ characteristics shown in Figure 4.16. Although there is good correlation between modeled and measured

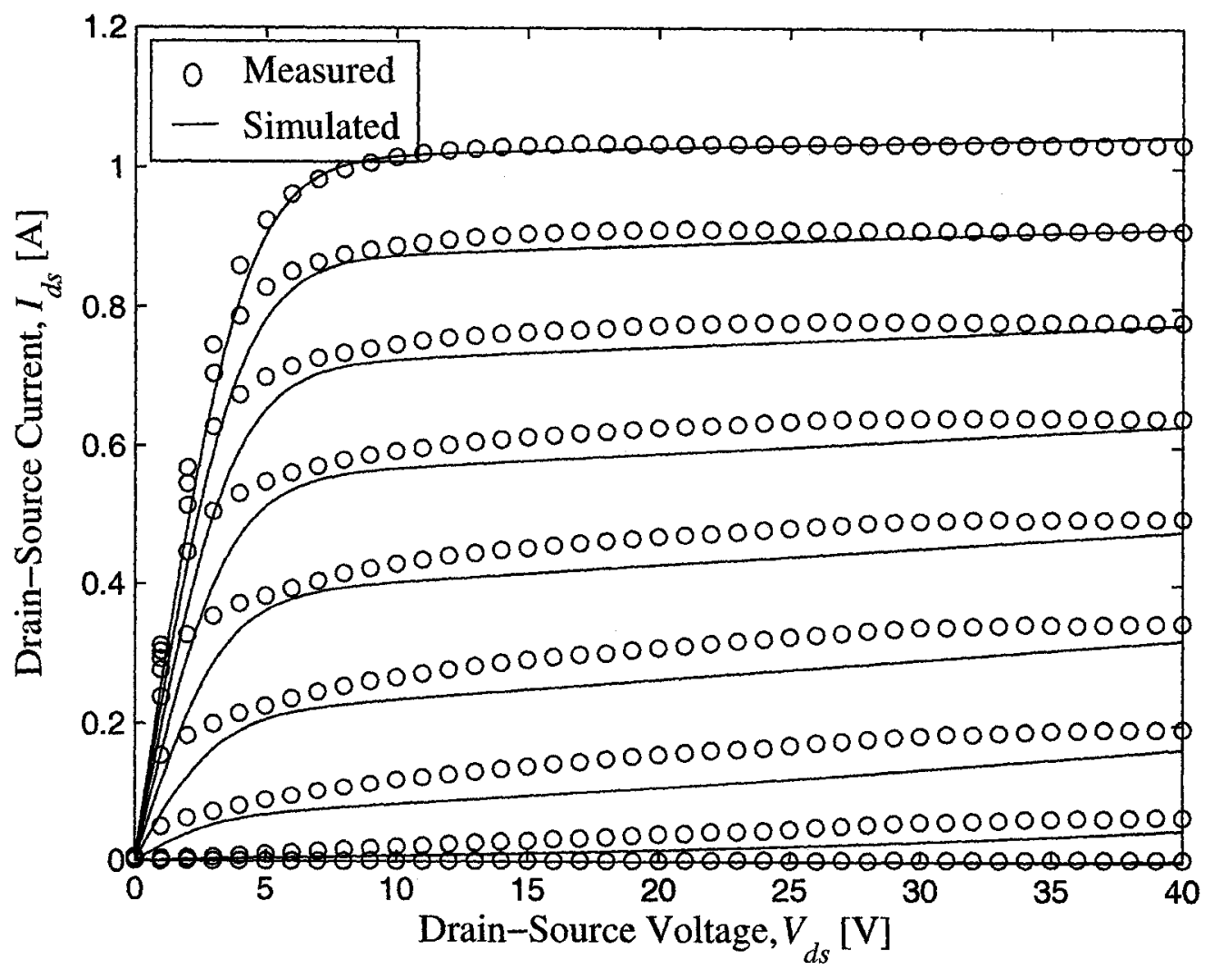

Figure 4.16: Comparison of modeled vs. measured $I_{d s}$ vs. $V_{d s}$ 
data, the model does not capture the knee region of the $I_{d s}-V_{d s}$ profile over most of the $V_{g s}$ plane. The measured data indicates a smaller on-resistance than that predicted by the model, translating to higher measured efficiency levels than those predicted in Class E PA circuit simulations.

Figure 4.17 shows measured DC $g_{m}$ and $I_{d s}$, plotted versus $V_{g s}$, and overlayed with simulations, for $V_{d s}$ ranging from $10 \mathrm{~V}$ to $40 \mathrm{~V}$. Modeled predictions are in good agreement with measured device performance.

Measured and simulated small-signal s-parameter data for $V_{d s}=20 \mathrm{~V}$ and $V_{g s}=-1$ $\mathrm{V}$ are shown in Figure 4.18. This level of agreement is typical across drain bias levels ranging from $10 \mathrm{~V}$ to $40 \mathrm{~V}$ and gate bias levels from below pinch-off $(-4 \mathrm{~V})$ to $0 \mathrm{~V}$. The only exceptions are at or near $V_{p}$ and near the gate voltage that drives the Schottky diode into the forward biased region of operation. The $S_{11}$ and $S_{22}$ responses in Figure 4.19 highlight the discrepancies in the model for a fixed $V_{d s}$ of $20 \mathrm{~V}$.

With $V_{g s}$ set to $1 \mathrm{~V}$, measurements in Figure 4.19 (a) show that the input resistance is constant with frequency, while the model predicts a frequency dependence. Similarly, the model predicts a frequency dependent output resistance at $V_{g s}=-3 \mathrm{~V}$, a prediction that is contrary to the measured response as shown in Figure 4.19(b). These deviations are a consequence of transmission line effects of the gate, source, and drain fingers on the die that are not captured by the device model [30]. The choice of ideality factor is also to blame (see Table 4.5). Although $\eta$ is not particularly high at 2.05, the physical process that determines $\eta$, dictated by the Sah-Noyce-Schokley theory, does not account for ideality factors greater than 2.0 [71]; but ideality factors as high as 26 have been encountered 

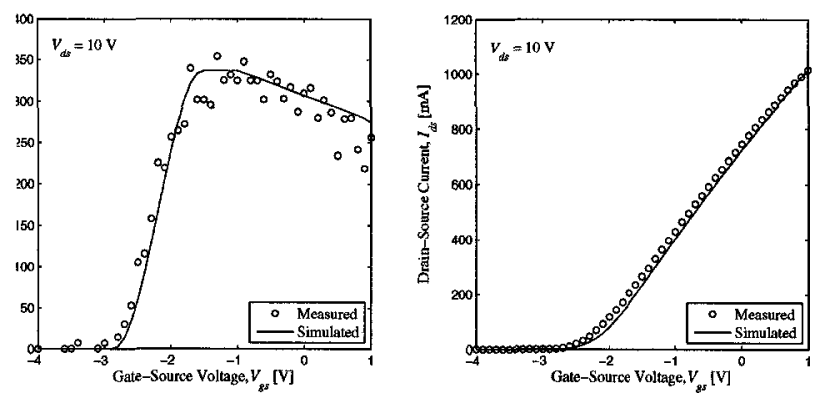

(a)
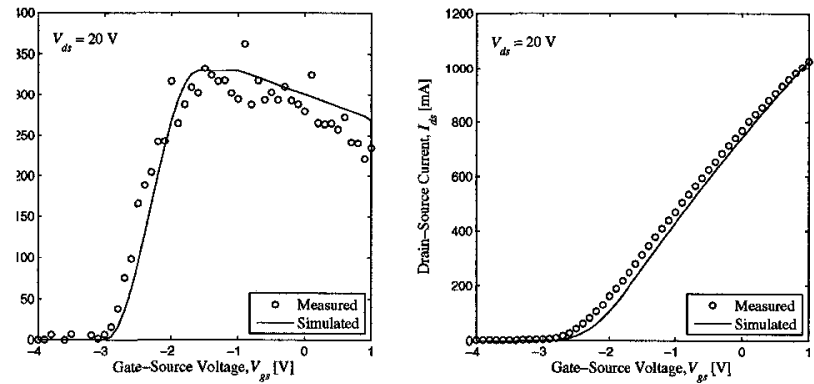

(b)
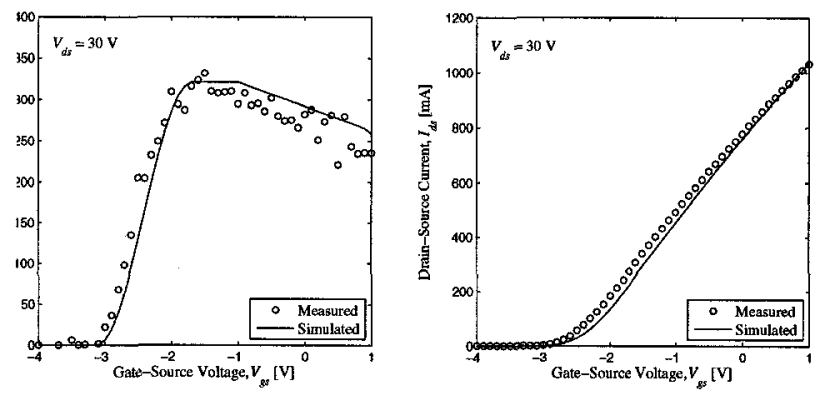

(c)
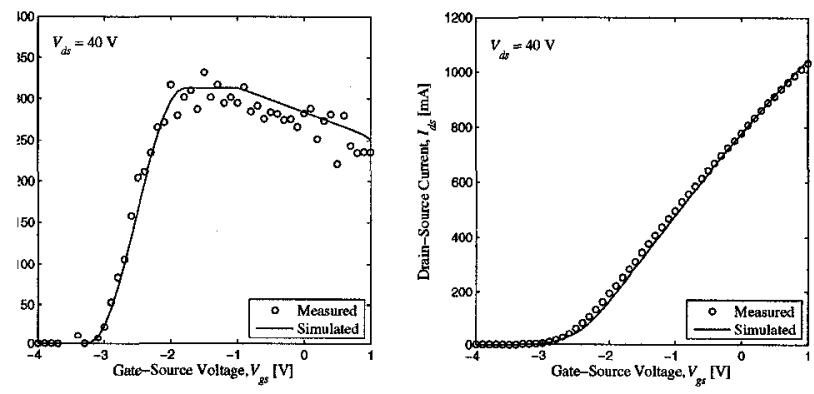

(d)

Figure 4.17: Comparison of measured and simulated DC $g_{m}$ and $I_{d s}$ with respect to $V_{g s}$ for (a) $V_{d s}=10 \mathrm{~V}$, (b) $V_{d s}=20 \mathrm{~V}$, (c) $V_{d s}=30 \mathrm{~V}$, and (d) $V_{d s}=40 \mathrm{~V}$. 

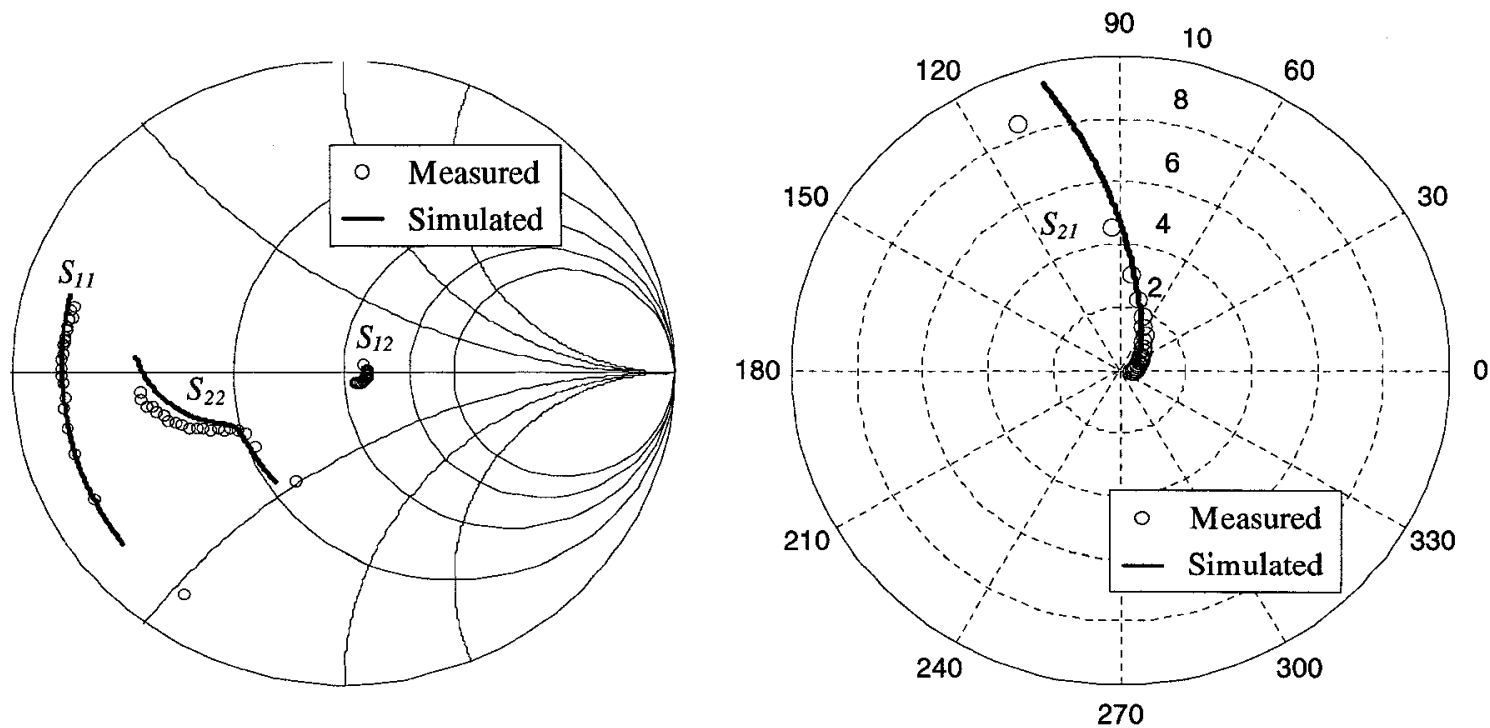

Figure 4.18: Comparison of measured vs. simulated s-parameters for $V_{d s}=20 \mathrm{~V}$ and $V_{g s}=-1 \mathrm{~V}$.

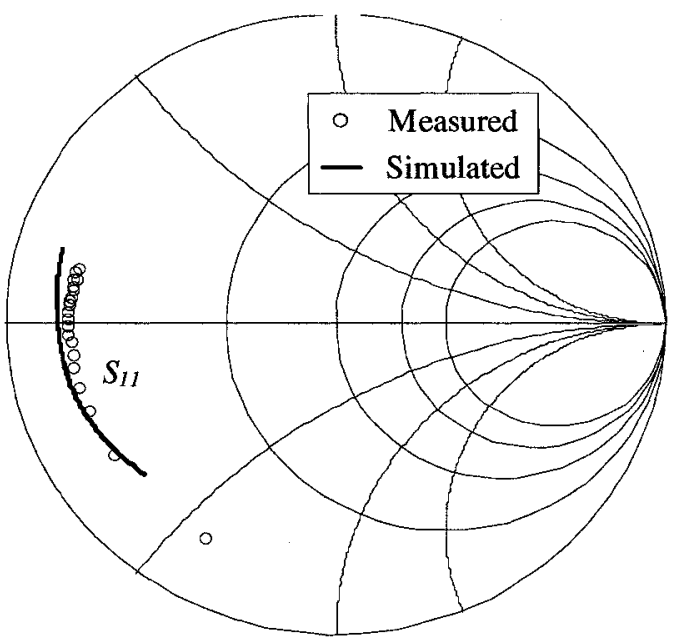

(a)

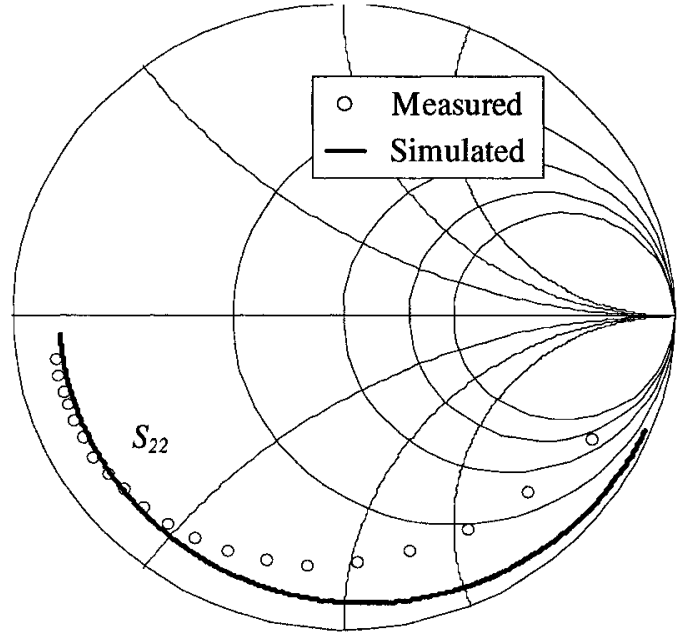

(b)

Figure 4.19: Nonlinear model response deviates from measurements for (a) $V_{g s}=1 \mathrm{~V}$ and (b) $V_{g s}=-3 \mathrm{~V}$. The behaviour of the GaN Schottky diode and the transmission line nature of the gate, drain, and source fingers are not captured, causing deviations in predicted HEMT performance near $V_{p}$ and the forward-biased gate voltage. 
for the GaN HEMT in literature [72]. Evidently, the high ideality factor in the GaN Schottky diode is a common occurrence that requires a detailed study. Figure 4.20 shows the measured and simulated responses of the Schottky diode for further confirmation of the reason for the deviations.

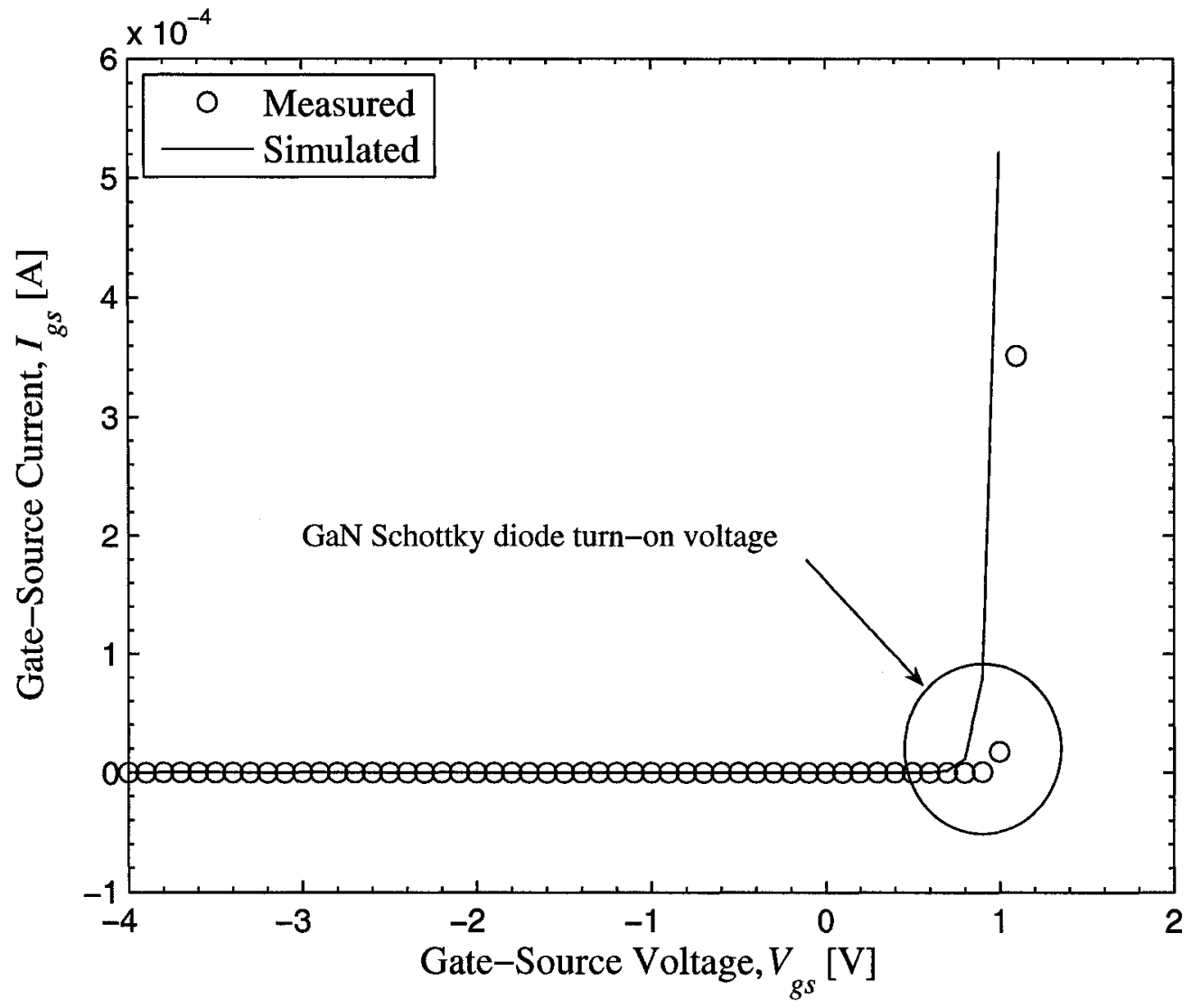

Figure 4.20: Simulated vs. measured GaN Schottky gate characteristic.

Measured load pull data for this $2 \mathrm{~mm}$ die are shown in Figure 4.21. Under Class A bias conditions $\left(V_{d s}=20 \mathrm{~V}\right.$ and $\left.V_{g s}=-1 \mathrm{~V}\right)$, measurements show that an optimum $P A E$ of $23.94 \%$ is reached for a gain of $6.75 \mathrm{~dB}$ and an output power of $31.75 \mathrm{dBm}$. Although not exact, simulations predict large-signal power characteristics of the device with some level of certainty. 


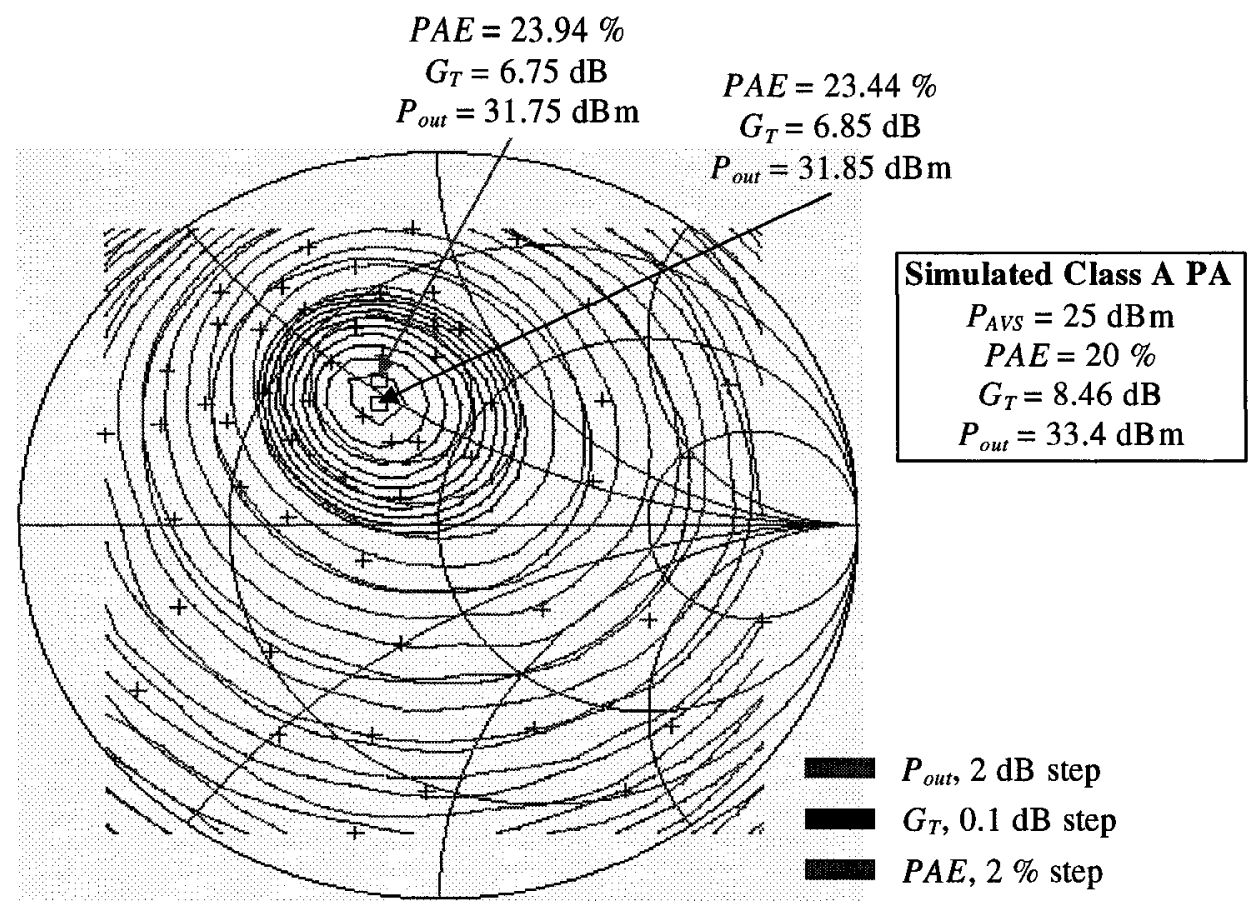

Figure 4.21: Measured load pull contours of the $2 \mathrm{~mm} \mathrm{GaN} \mathrm{HEMT} \mathrm{die} \mathrm{operating} \mathrm{at} 3.5$ $\mathrm{GHz}$ and tuned for an input power of $25 \mathrm{dBm}$ at a Class $\mathrm{A}$ bias level.

The parameter extraction procedure is based on published technical theory and corresponds to the process flow indicated in the model's documentation. The fact remains however, that the model generates an approximate mathematical fit to measured device behaviour. The model, during its conception, was not tested on GaN HEMTs due to their relative newness, so it is conceivable that some of the model's equations simply cannot predict the physical properties of $\mathrm{GaN}$ devices.

\subsection{Summary}

The switching behaviour of an active device in a Class E PA cannot be treated as ideal at microwave frequencies. Transistor parasitics and non-ideal driving waveforms need to be considered in the design process. The active device which, in this case, is a new GaN-on-Si 
HEMT, is modeled in order to accurately capture the performance of a nonlinear PA in simulation.

An existing ADS-based GaAs HEMT model is used to predict the performance of a $2 \mathrm{~mm}$ GaN-on-Si HEMT based on measured data supplied by the manufacturer of the device. The model predicts measured device behaviour for DC power up to $20 \mathrm{~W}$ and $I_{d s}$ greater than $1 \mathrm{~A}$. The model is accurate for frequencies up to $20 \mathrm{GHz}$. Correlation between measured and simulated device behaviour is weaker near the gate pinch-off and forward biased regions suggesting that some modifications to the model are needed for this $\mathrm{GaN}$ process.

The empirical model does not allow modification of the mathematical equations that define the model. This, combined with the newness of the technology, limits the ability of the model to provide an exact representation of the physical device. In spite of its deficiencies, the model predicts measured device behaviour reasonably well for the purposes of this work. 


\section{Chapter 5}

\section{Class E GaN-on-Si Microwave Power Amplifier}

Given the high breakdown voltage and high current density of GaN HEMTs, a Class E power amplifier is a natural progression of the work presented so far. Using the nonlinear model presented in the previous chapter, the first known $3.5 \mathrm{GHz}$ GaN-on-Si Class $\mathrm{E}$ power amplifier is demonstrated in this chapter. The work presented here is an adaptation of the Class E PA analysis and design done by Thomas Mader in his Ph.D. thesis [38, 73]. His work has been carried forward over the years [74-76], but to the best of the author's knowledge, has never been implemented in a GaN-on-Si PA. The GaN-based Class E switched-mode concept is not novel. Others have published working Class E power amplifiers, but most have based their $\mathrm{GaN}$ technology on expensive $\mathrm{SiC}$ substrates. Perhaps the single most distinguishing feature of the PA in this work, is that the active device has a silicon substrate; a technology that combines the superior performance of GaN with a mass-producible inexpensive substrate.

Starting with the ideal Class E power amplifier circuit, this chapter evolves the basic 
lumped element design into a planar transmission line circuit. As the chapter progresses, the degree of "idealness" is removed from the circuit simulations until a practical design of each component in the PA is developed. The PA design specifications established in Chapter 3 are repeated in Table 5.1, along with substrate properties.

\begin{tabular}{|c|c|}
\hline Design frequency $\left(f_{1}\right)$ & $3.5 \mathrm{GHz}$ \\
\hline Output power $\left(P_{1}\right)$ & $1 \mathrm{~W}$ \\
\hline Power-added efficiency $(P A E)$ & $\geqslant 40 \%$ \\
\hline Minimum input return loss $(R L)$ & $15 \mathrm{~dB}$ \\
\hline Drain bias supply voltage $\left(V_{d d}\right)$ & $25 \mathrm{~V}$ \\
\hline \hline Material & Rogers RT/duroid@ 5880 \\
\hline Substrate thickness & 31 mil \\
\hline Cladding & 1 oz. rolled copper foil \\
\hline Dielectric constant & 2.20 \\
\hline Dissipation factor $(10 \mathrm{GHz})$ & 0.0009 \\
\hline
\end{tabular}

Table 5.1: Class E PA specifications and high frequency laminate material properties.

Figure 5.1 shows a high-level diagram of the Class E PA, which consists of input and output matching networks and additional biasing circuitry. The phasor impedance

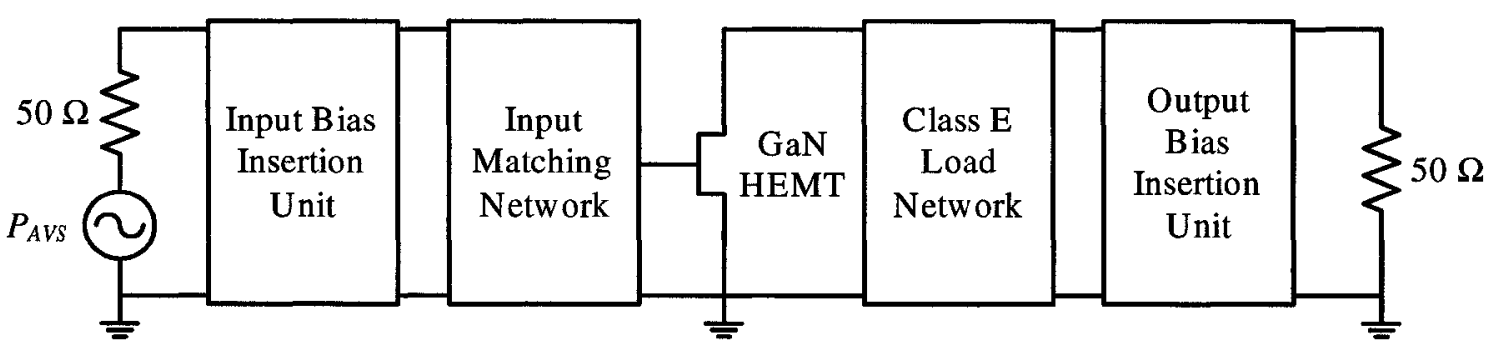

Figure 5.1: Block diagram of Class E power amplifier module

of the Class E load network is used to synthesize the appropriate output load network that meets the criteria for Class $\mathrm{E}$ operation. The bias insertion circuit, which must be included as part of the matching networks, is carefully designed to function only to bias the active device without causing any unwanted effects. An entire section is duly devoted 
to the design of the input and output biasing circuits. And finally, when dealing with appreciable power levels, thermal effects cannot be ignored, so the chapter also includes a heat sink design that ensures the best available thermal gounding to the GaN HEMT.

\subsection{Ideal Class E Power Amplifier}

The basic Class E power amplifier proposed by the Sokals, and whose theoretical performance is discussed in Chapter 3, is shown again in Figure 5.2. The design is fairly

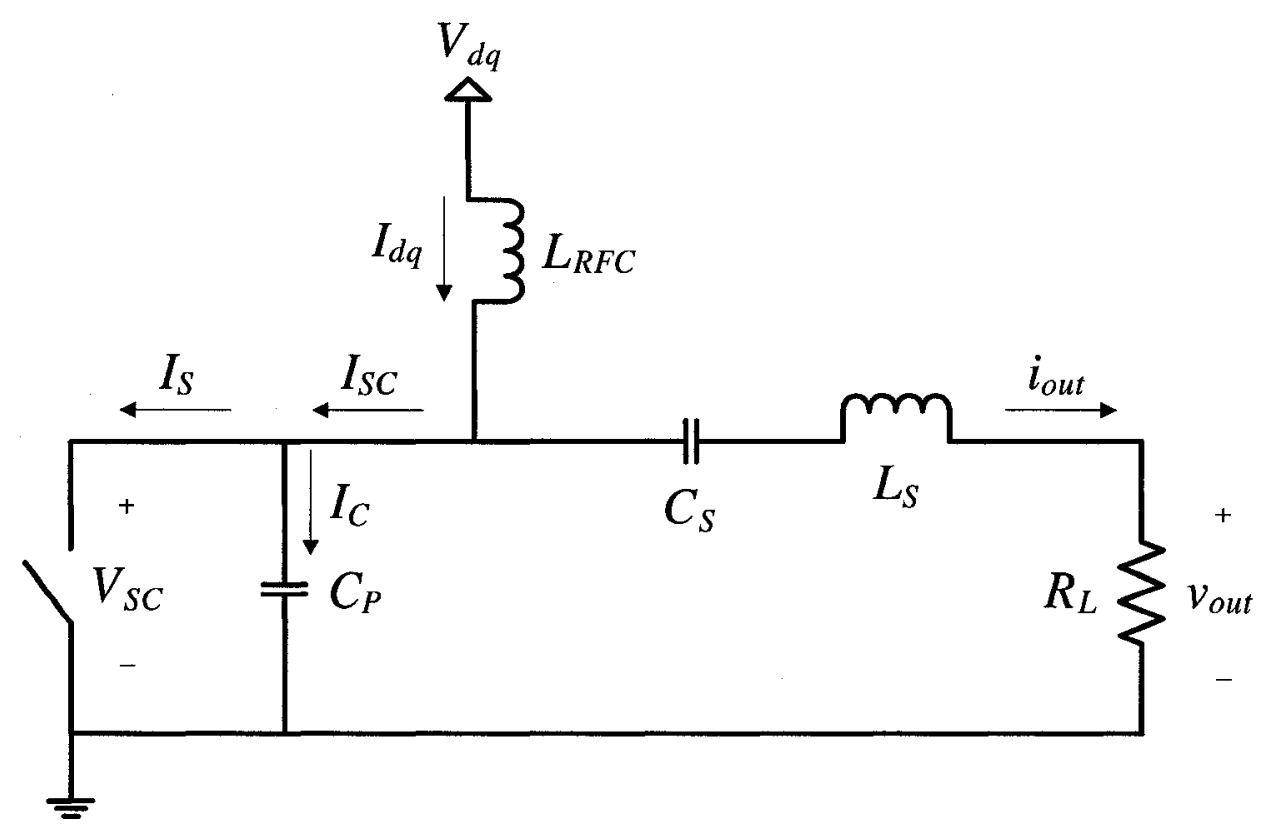

Figure 5.2: Original Class E PA used to verify ideal Class E operation in simulation.

straightforward: one must have fundamental frequency, device breakdown voltage, and loaded Q-factor $\left(Q_{L}\right)$ readily available to apply to the design equations presented by Nathan Sokal in [33]. The drain supply voltage is $20 \mathrm{~V}$ putting the required transistor breakdown voltage and peak current to at least $71 \mathrm{~V}$ and $660 \mathrm{~mA}$, respectively, according to Equations 3.3.14 and 3.3.16. Of course, these are theoretical estimates; in practice, 
there are loss mechanisms in the circuit (see Section 3.3) that will reduce the peak voltage swing. The idealized output power level and circuit elements are computed using Equations 5.1.1 - 5.1.5 for $V_{d q}=20 \mathrm{~V}, Q_{L}=5$, and $R_{L}=50 \Omega$. The calculations yield $P_{\text {out }}=4.13 \mathrm{~W}, C_{P}=0.19 \mathrm{pF}, C_{S}=0.24 \mathrm{pF}, L_{S}=0.11 \mathrm{nH}$, and $L_{R F C}=326 \mathrm{nH}$.

$$
\begin{aligned}
\frac{P_{o u t} R_{L}}{V_{d q}^{2}} & =0.51659 \\
C_{P} \omega_{s} R_{L} & =0.20907 \\
C_{S} \omega_{s} R_{L} & =0.26924 \\
L_{S} & =\frac{Q_{L} R_{L}}{\omega_{s}} \\
L_{R F C} & =\frac{30}{\omega_{s}^{2} C_{P}}
\end{aligned}
$$

The simulations for the ideal model are shown in Figure 5.3. The drain voltage and current reach their respective peak values of approximately $71 \mathrm{~V}$ and $600 \mathrm{~mA}$. There are instantaneous spikes in current across the switch at the exact moment that the switch turns on that would normally appear as losses across any inductance present between the switch and $C_{P}$. It has been shown however, that for nonzero output power, at least one such discontinuity per cycle is necessary [77]. Note that the current spikes have been omitted from Figure 5.3 for clarity.

Although the ideal Class E PA presents a valid design strategy, the problem is that the required Class $\mathrm{E}$ shunt capacitance is calculated and cannot be independently chosen. The driving waveform is an additional concern in that a $3.5 \mathrm{GHz}$ square-wave input drive with a $50 \%$ duty cycle is difficult to implement. The next section shows a simple impedance 

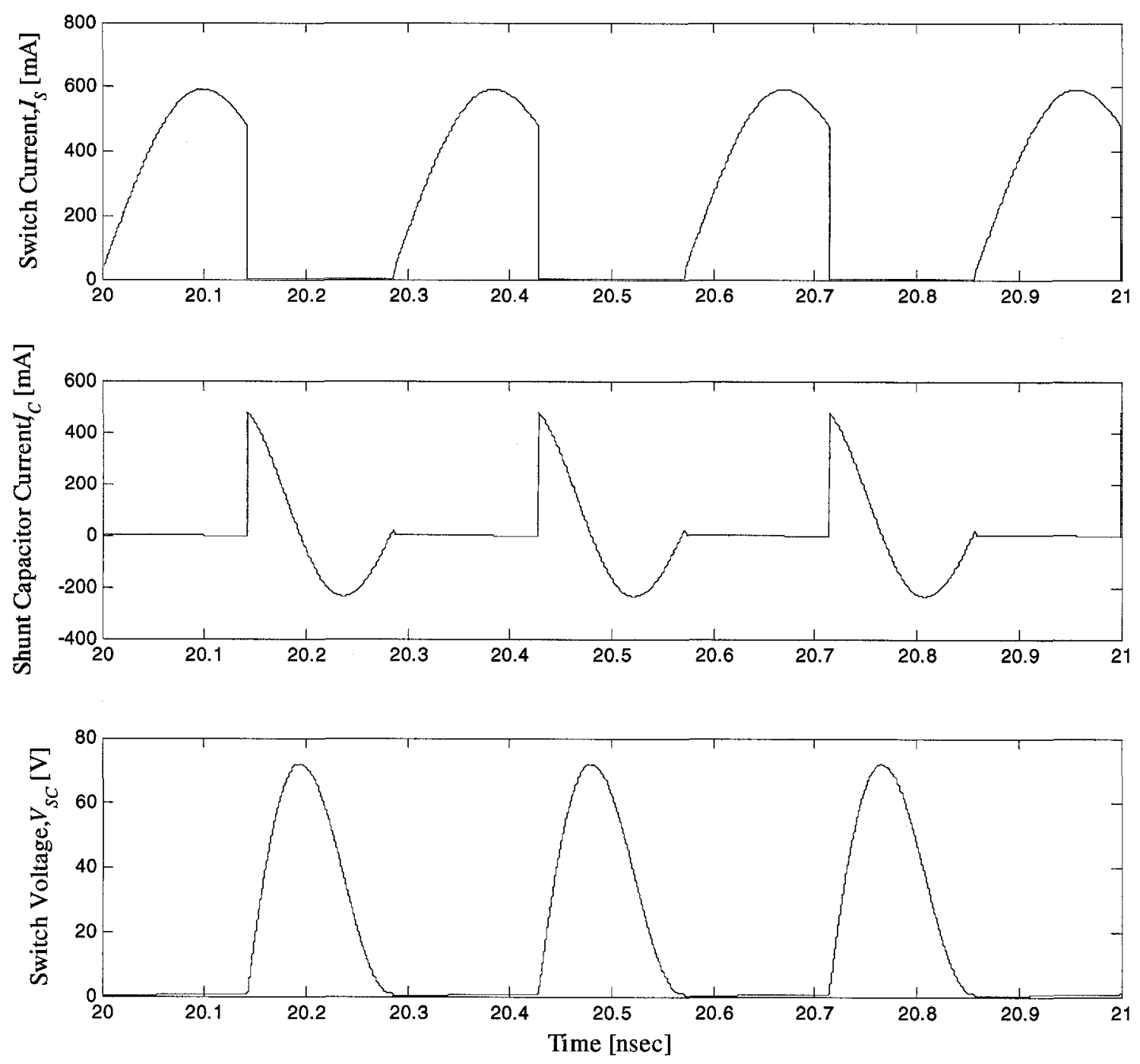

Figure 5.3: Simulated voltage and current waveforms verify ideal Class E PA operation. 
analysis that solves the latter problem and allows the load network to be realized with microstrip transmission lines.

\subsection{The Class E Load Network}

The purpose of the Class E load network is to

1. provide the correct impedance magnitude and load angle to the output of the HEMT at the fundamental frequency such that all Class E criteria, as per the discussion in Section 3.3, are satisfied; and

2. ensure high impedance terminations for all higher-order harmonics (this is implicit in the discussion in Section 3.3)

Although the first purpose is realizable, the second is not due to the narrowband nature of transmission line circuits. For the moment, the discussion continues with the assumption that higher-order harmonics are properly terminated.

The lumped element load network analysis proceeds with ideal RF chokes and coupling capacitors in place of the true input and output bias insertion circuits. The input and output biasing circuits are designed to not affect circuit performance, appearing invisible to the drain of the HEMT. This level of exclusivity can be maintained by the biasing circuits provided that the bias supply is properly decoupled and the bias circuit does not load the PA over the RF band of interest. The bias circuit is assumed to consist only of ideal elements throughout this Chapter until Section 5.4, where the complete design, including measurements, is demonstrated. 
A lumped element Class E load network, based on the phasor impedance required at the output of the transistor, is presented next. A microstrip transmission line implementation follows along with a discussion on harmonic terminations.

\subsubsection{Lumped Element Analysis}

Fourier series analysis of the Class E load network gives an expression for the fundamental component of $V_{S C}$ in Figure 5.2. This voltage, divided by the RF current in the load network, gives the RF phasor impedance as a function of switching frequency $\left(\omega_{s}\right)$ and shunt capacitance $\left(C_{P}\right)$,

$$
Z_{E} \approx \frac{0.28015}{\omega_{s} C_{P}} e^{j 49.0524^{\circ}}
$$

keeping in mind that $C_{P}$ is intended to be solely composed of the HEMT's output capacitance, $C_{d s o}$ [38]. The ultimate goal is to synthesize the impedance in Equation 5.2.1 using microstrip transmission lines. So, as a next step in the analysis, this impedance is shown schematically in Figure 5.4. The Class E load network is composed of lumped elements

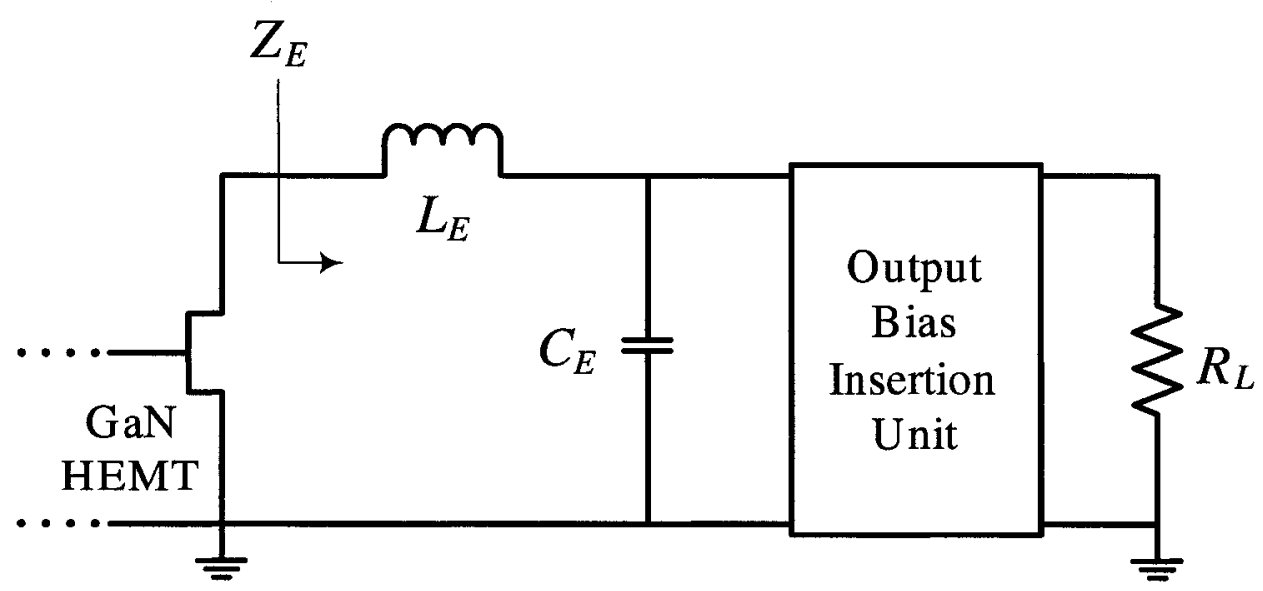

Figure 5.4: The load, $R_{L}$, is technically transformed through the output bias circuit as well as the Class $\mathrm{E}$ load network. 
and the output bias insertion unit is assumed to not affect the RF performance of the circuit. The output bias circuit is effectively invisible to the transistor, so the transistor actually "sees" the circuit in Figure 5.5. The input impedance of the load network is

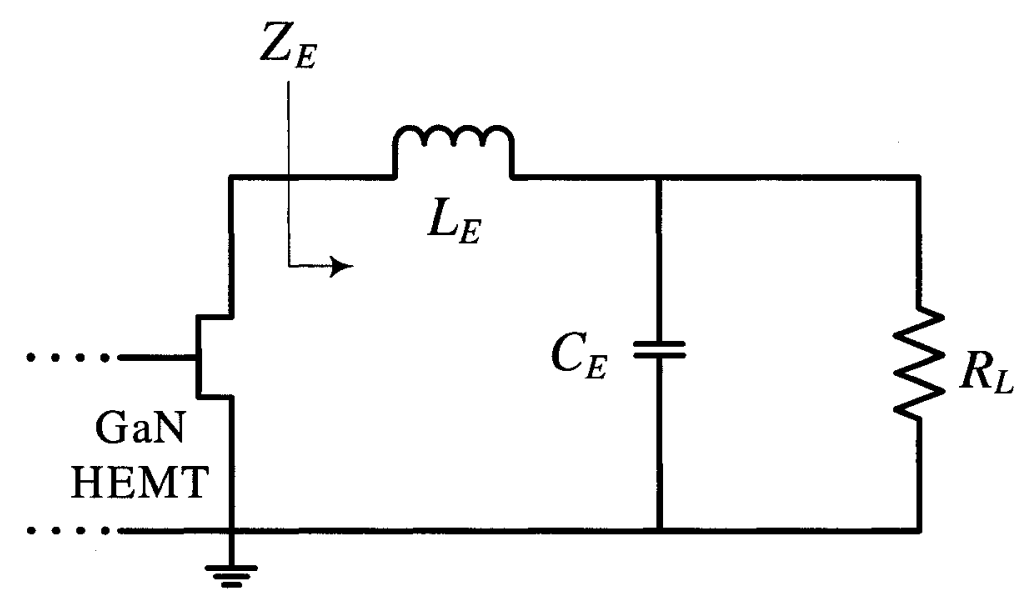

Figure 5.5: When designed correctly, the biasing circuitry does not load the output of the transistor in the RF band of interest; instead, it appears invisible to the circuit.

$$
Z_{E}=j \omega_{s} L_{E}+\frac{R_{L}}{1+j \omega_{s} C_{E} R_{L}}
$$

Equating this load network impedance to the required Class E phasor impedance in Equation 5.2.1 gives expressions for $L_{E}$ and $C_{E}$ [38]:

$$
\begin{gathered}
L_{E}=\frac{k_{0}}{\omega_{s}^{2} C_{p}}\left(\sin \theta_{0}+\cos \theta_{0} \sqrt{\frac{\omega_{s} C_{p} R_{L}}{k_{0} \cos \theta_{0}}-1}\right) \\
C_{E}=\frac{1}{\omega_{s} R_{L}} \sqrt{\frac{\omega_{s} C_{p} R_{L}}{k_{0} \cos \theta_{0}}-1}
\end{gathered}
$$

where $k_{0}=0.28015, \theta_{0}=49.0524^{\circ}, R_{L}$ is the load resistance, and $C_{P}$ is the required capacitance in parallel with the output of the HEMT. The expressions in Equations 5.2.3 and 5.2.4 give $\omega_{s}, R_{L}$, and $C_{P}$ as independent variables that can be freely chosen. This means that, once the switching frequency and output power are known, a suitable transistor can be chosen whose intrinsic output capacitance can be used, in its entirety, as 
the required shunt capacitance for Class $\mathrm{E}$ operation. Keep in mind that $C_{P}$ and $C_{d s o}$ are assumed to be linear. In actual fact, Figure 4.15 shows that $C_{d s o}$ depends on $V_{d s}$ making it nonlinear. Further to that, it should be noted that output power is directly a function of the drain bias voltage $\left(V_{d q}\right)$ as per the relation in Equation 5.1.1, but is indirectly related to Equation 5.2.1 in that the correct impedance must be present for Equation 5.1.1 to be true.

Before moving on to the transmission line circuit, the preceding analysis is verified in conjunction with the GaN HEMT nonlinear model. Figure 5.6 shows a Class E PA schematic complete with the GaN HEMT and a lumped element load network. The

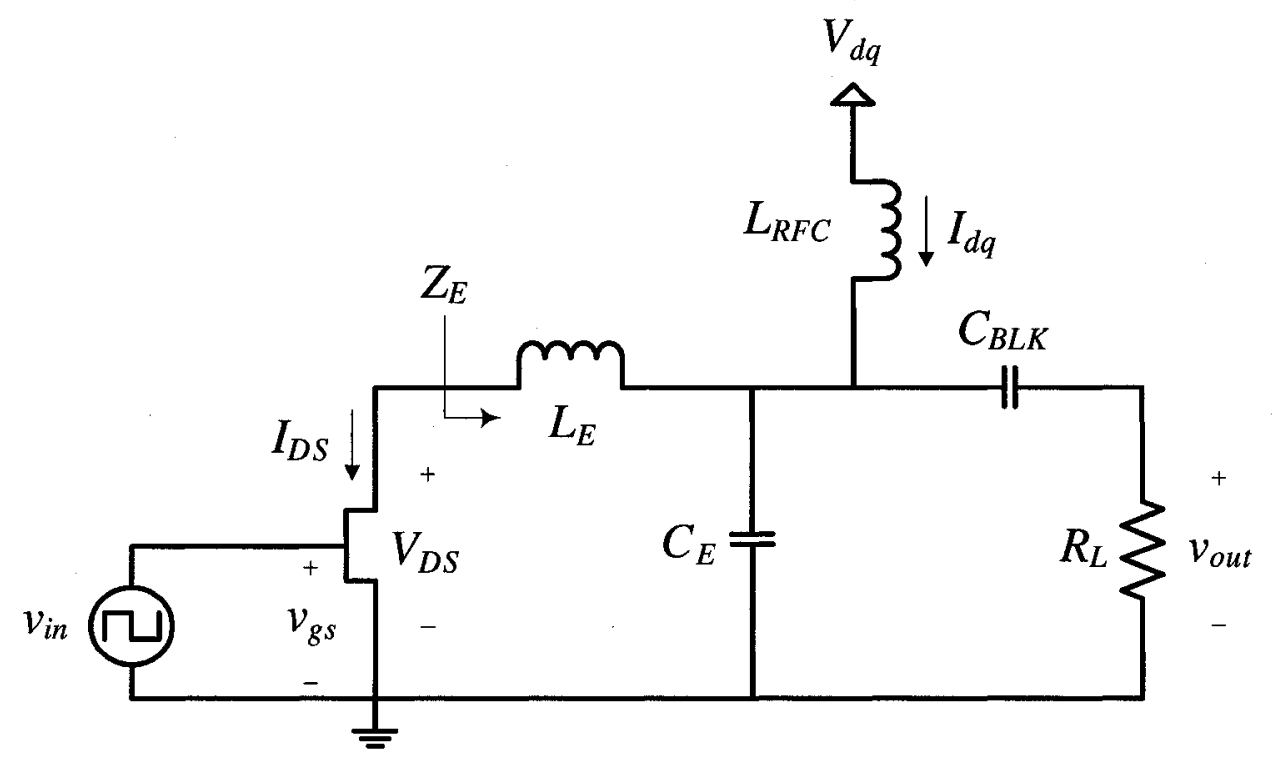

Figure 5.6: Class E PA with nonlinear GaN HEMT device model and ideal lumped element load network that transforms the $50 \Omega$ to the phasor impedance $Z_{E}$.

gate of the HEMT is switched from $V_{g s, o f f}=-4 \mathrm{~V}$ to $V_{g s, o n}=1 \mathrm{~V}$ by an ideal driving circuit that produces a $50 \%$ duty cycle square-wave voltage reference (indicated by $v_{\text {in }}$ in Figure 5.6) at the design frequency. Ideally, the minimum power needed to switch the 
device should be used to maximize gain, suggesting that a switching voltage $\left(V_{S W}\right)$ of about $4 \mathrm{~V}$ (instead of $5 \mathrm{~V}$ ) is adequate since the gate pinch-off voltage is around $-3 \mathrm{~V}$. For this work, the importance of gain is second to that of drain efficiency.

The load impedance and the lumped elements in the load network are computed using Equations 5.2.1, 5.2.3 and 5.2.4. The capacitance, $C_{P}$, is set to the HEMT's output capacitance, $C_{d s o}$, which is $0.28 \mathrm{pF}$ (see Appendix A). As in the ideal Class E PA example, the switching frequency $\left(f_{s}\right)$ and load impedance $\left(R_{L}\right)$ are $3.5 \mathrm{GHz}$ and $50 \Omega$, respectively. Making the appropriate substitutions into Equation 5.2 .1 gives $Z_{E}=29.817+j 34.364 \Omega$. The lumped elements are also determined: $L_{E}=2.678 \mathrm{nH}$ and $C_{E}=0.748 \mathrm{pF}$.

Figure 5.7 shows $V_{D S}$ superimposed by the gate driving voltage, $v_{g s}$. Comparing this to the ideal case in Figure 5.3, it is clear that the Class E conditions are not being satisfied. The on-state resistance of the HEMT is approximately $4 \Omega$ from the device's measured $I_{d s}$ vs. $V_{d s}$ curves in Figure 4.16. So when in the on state, this finite resistance reduces drain efficiency by prohibiting $V_{D S}$ from reaching $0 \mathrm{~V}$. In addition, the HEMT turns on before $V_{D S}$ reaches its minimum value, which creates a short-circuit across the output capacitance $\left(C_{d s o}\right)$, causing further losses as the capacitor's stored energy is discharged through the HEMT. The current through the HEMT is composed of the current that flows through the transistor itself when in the on-state and that which flows through $C_{d s o}$ in the transistor's off-state. A plot with $I_{D S}$ together with $V_{D S}$ is shown in Figure 5.8. Since a real transistor is being used, it is difficult to establish the moment in time that $I_{D S}$ transitions from the $4 \Omega$ on-state resistance to $C_{d s o}$. Taking a simplistic approach to the analysis, let us say that the device transitions back-and-forth from the on- to the 


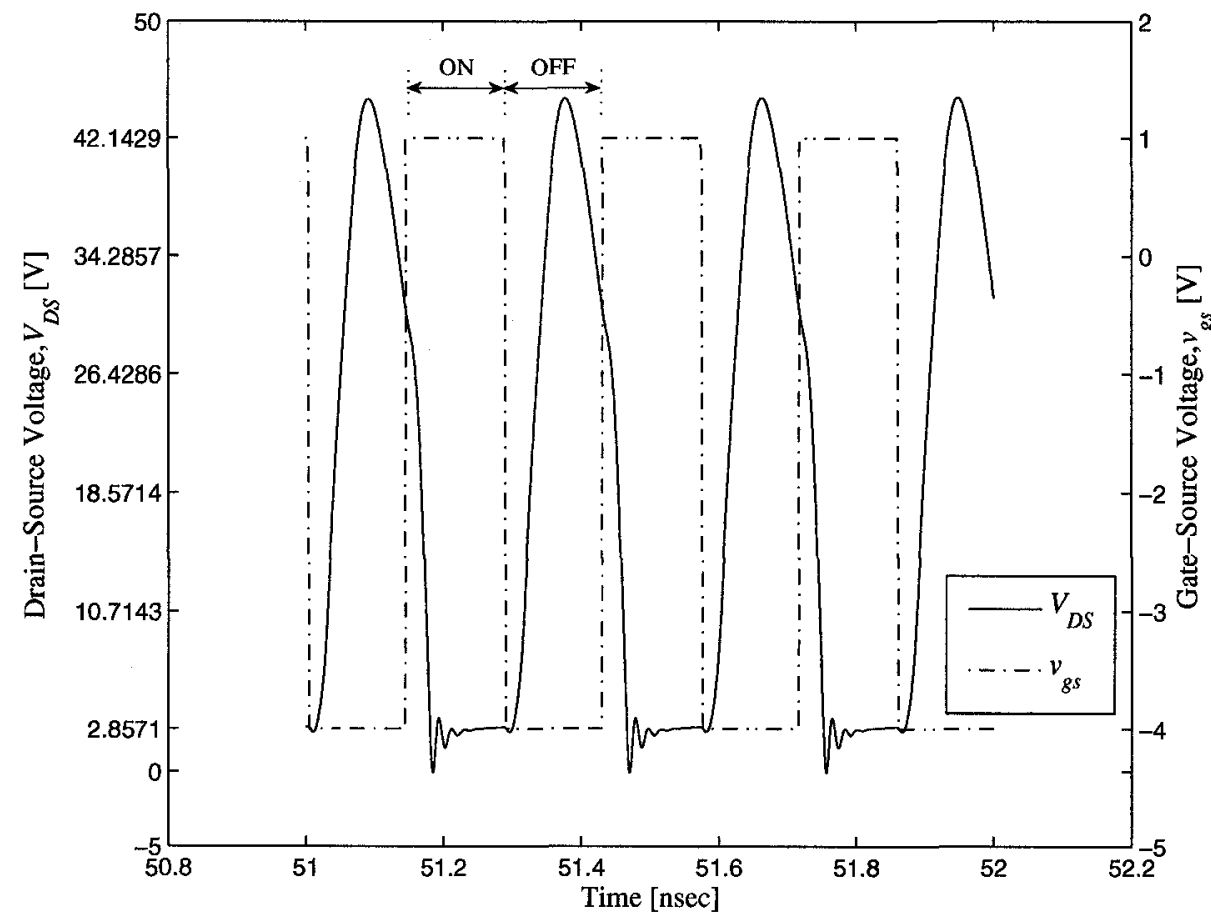

Figure 5.7: An ideal gate driving signal produces a Class E drain voltage when the drain of the GaN HEMT is loaded by the phasor impedance $Z_{E}$. 
off-states instantaneously. The shaded region of the plot shows the power that is lost every period due to the short-circuit across $C_{d s o}$. Since, in the off-state, the plot indicates that $I_{D S}$ flows entirely through $C_{d s o}$, there is no power loss, which is likely not the case in practice.

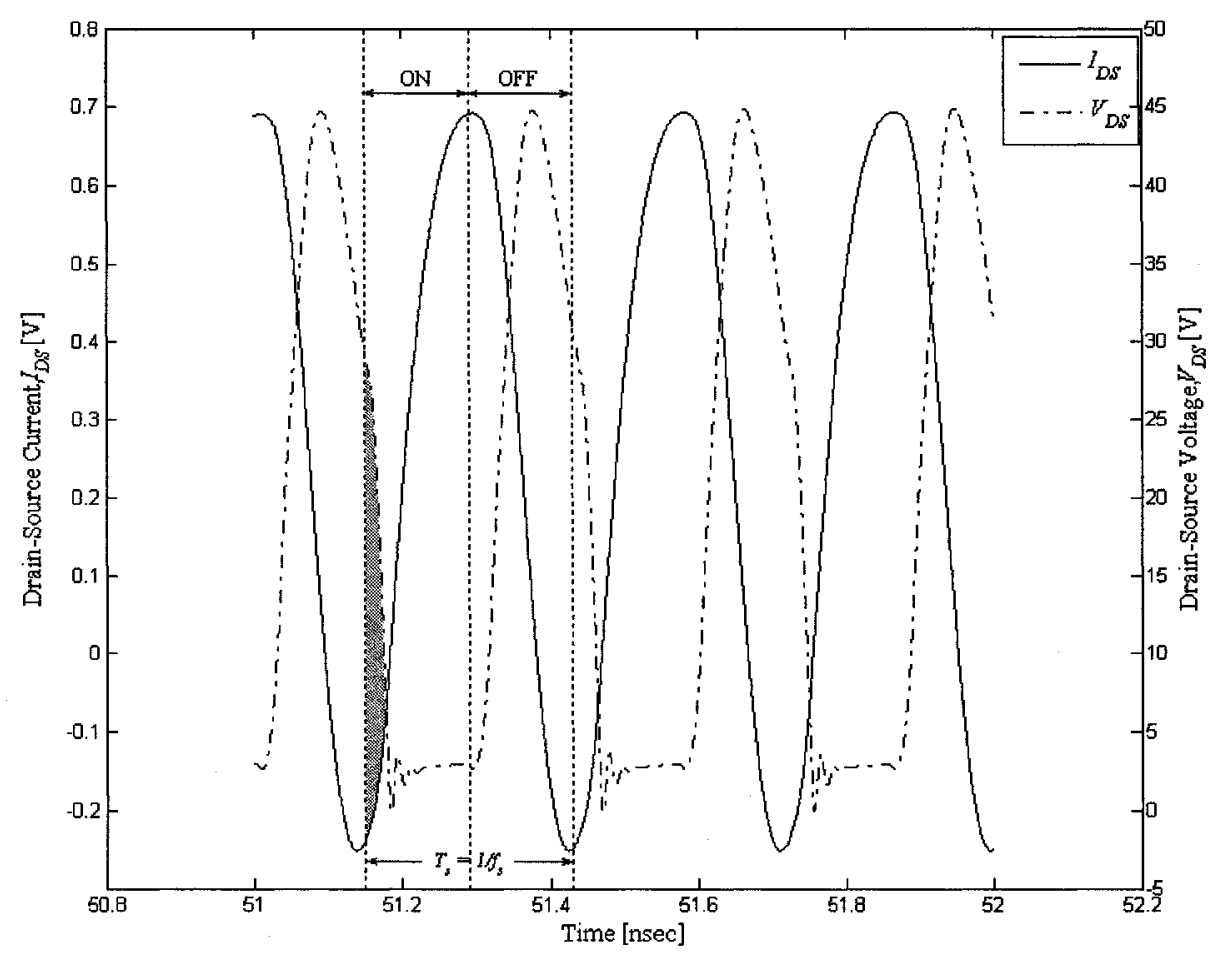

Figure 5.8: An ideal gate driving signal produces these $I_{D S}$ and $V_{D S}$ waveforms when the drain of the GaN HEMT is loaded by the phasor impedance $Z_{E}$.

There is room for improvement in terms of the circuit's response to an ideal squarewave input. The Class E impedance given by Equation 5.2.1 needs adjustment to account for bondwire inductance and device parasitics. As a logical next step, the load network impedance is adjusted to maximize drain efficiency and the ideal $3.5 \mathrm{GHz}$ square-wave driver is replaced with a large-amplitude voltage source conjugately matched to the gate of the $2 \mathrm{~mm}$ HEMT. Relative to the output, the input of a transistor is less sensitive to 
the type of matching circuit presented to the gate. Although a small-signal input match is a good first-order approximation [26], the gate of the HEMT is eventually power matched to the $50 \Omega$ source impedance to finalize the design (see Section 5.3).

This next stage in the design process is shown by the slightly more detailed schematic in Figure 5.9. The RF power required to switch the device from completely off (where $V_{g s, o f f}$

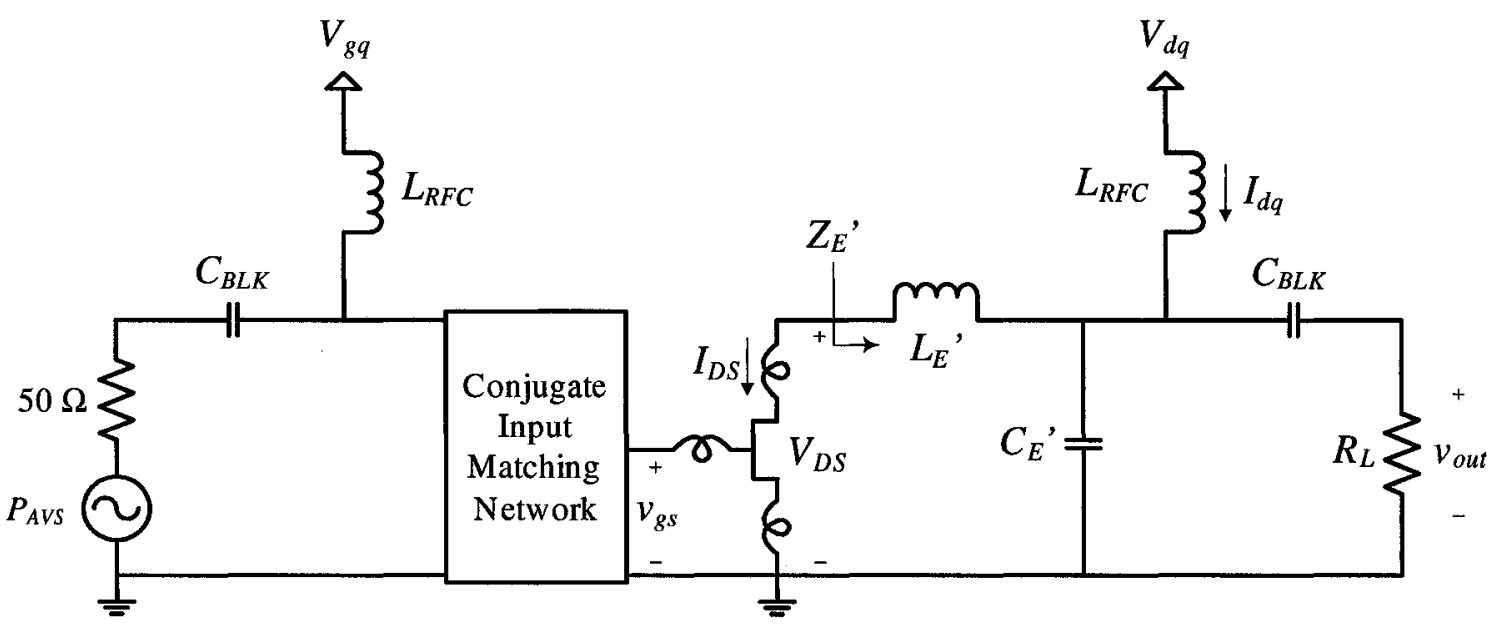

Figure 5.9: Lumped element Class E PA with tuned load network, small-signal matching circuit, and large-amplitude sinusoidal voltage input drive.

is $-4 \mathrm{~V}$ ) to completely on (where $V_{g s, o m}$ is $1 \mathrm{~V}$ ) is determined based on Equation 3.3.17. Taking the difference between $V_{g s, o n}$ and $V_{g s, o f f}$ gives $V_{S W}=5 \mathrm{~V}$. Setting $C_{g s}$ to $3.4 \mathrm{pF}$ from Figure 4.12, the required sinusoidal input power at $3.5 \mathrm{GHz}$ is approximately 25 $\mathrm{dBm}$ :

$$
P_{S W}=3.4(10)^{-12}(5)^{2} 3.5(10)^{9}=298 \mathrm{~mW} \text { or } 24.7 \mathrm{dBm}
$$

Tuning $L_{E}^{\prime}$ and $C_{E}^{\prime}$ in the load network for best drain efficiency at $P_{A V S}=25 \mathrm{dBm}$ yields the corrected phasor impedance, $Z_{E}^{\prime}$ (Equation 5.2.6), where $L_{E}^{\prime}$ and $C_{E}^{\prime}$ are tuned to 2.82 $\mathrm{nH}$ and $1.12 \mathrm{pF}$, respectively.

$$
Z_{E}^{\prime}=19.894+j 37.489 \Omega
$$


The adjusted impedance, $Z_{E}^{\prime}$, now forms the basis for the microstrip implementation, which is discussed next.

\subsubsection{Microstrip Transmission Line Implementation}

The ideal Class E PA assumes that all higher order harmonics see a high impedance load. In practice, it has been shown that only two harmonics are sufficient at the dran for approximate Class E operation [38]. An approximation is all that can be hoped to be achieved since neither the load network nor the input driving waveform imposes ideal conditions to the transistor.

The microstrip Class E power amplifier uses a two-stage load network whose function is to

1. present $Z_{E}^{\prime}$ to the drain of the HEMT at the fundamental frequency $(3.5 \mathrm{GHz})$, and

2. present an open-circuit condition to the the second harmonic $(7 \mathrm{GHz})$

This is illustrated in Figure 5.10. The high impedance second harmonic termination is acheived by setting the lengths of transmission line closest to the drain of the device to $45^{\circ}$ at the fundamental frequency. At the second harmonic, the electrical length of the lines is $90^{\circ}$, making them quarter-wave impedance transformers. The open-circuit at 'A' is transformed into a short-circuit at ' $\mathrm{B}$ ' and back to an open-circuit at ' $\mathrm{C}$ ' where the transmission line interfaces to the drain of the HEMT. Having properly terminated the second harmonic with the first stage of the load network, the second stage transforms the $50 \Omega$ load impedance to $Z_{E}^{\prime}$ at the fundamental frequency as seen at the output of the HEMT at ' $\mathrm{C}$ '. 


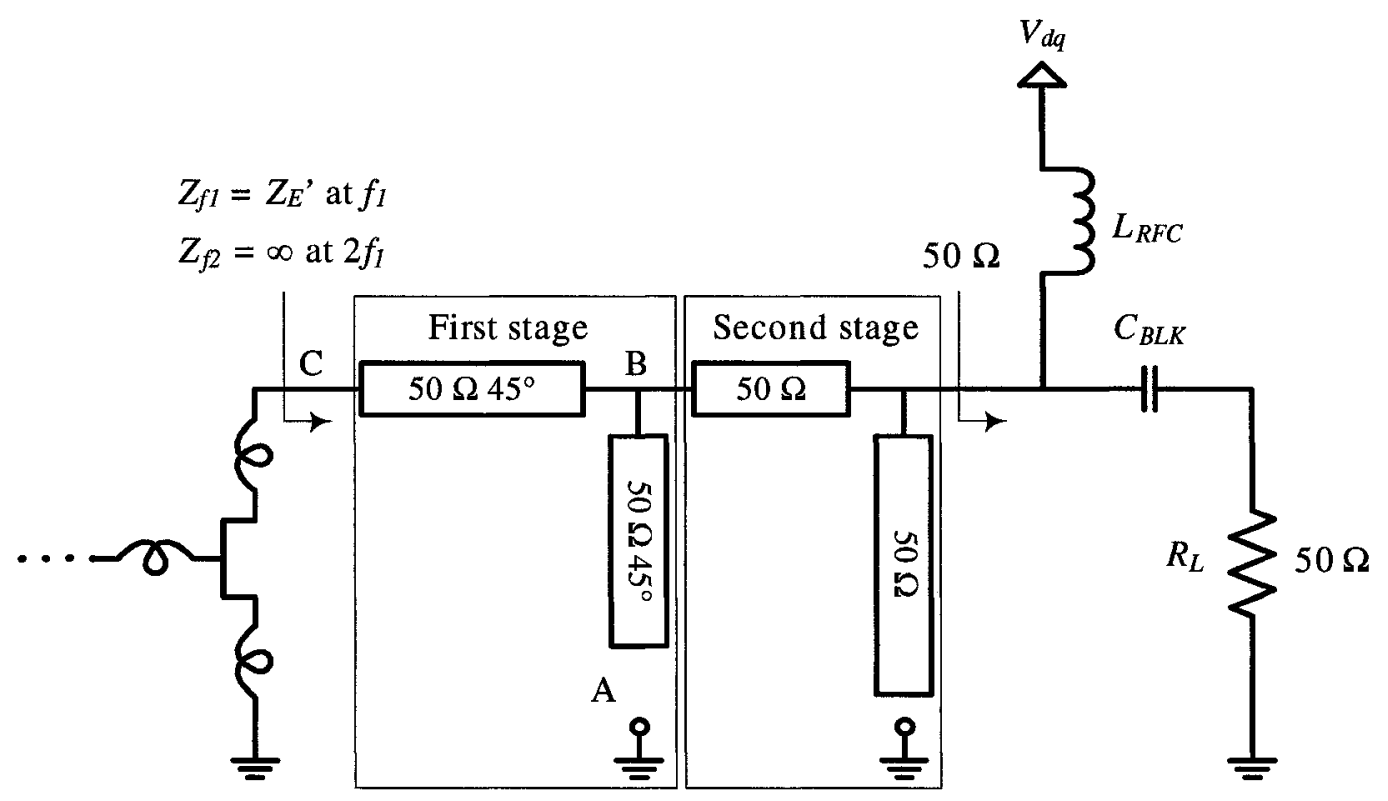

Figure 5.10: A two-stage load network is used at the output of the GaN HEMT to provide a high impedance termination to $2 f_{1}$ and $Z_{E}^{\prime}$ to $f_{1}$, where $f_{1}=3.5 \mathrm{GHz}$.

In the practical implementation shown in Figure 5.11, transmission line lengths and characteristic impedances have to be adjusted so that the impedance requirements at 3.5 $\mathrm{GHz}$ and $7 \mathrm{GHz}$ are met simultaneously. The simulated input impedance in Figure 5.12 shows that the impedance conditions are indeed satisfied: the second harmonic sees an open-circuit and the fundamental sees $Z_{f 1}=19.75+j 37.40 \Omega$, which is close to $Z_{E}^{\prime}$ in Equation 5.2.6. The true test of the load network design is demonstrated in Section 5.6, where high-efficiency switched-mode PA operation is verified in both simulations and measurements.

\subsection{Input Matching Network}

Since an RF signal source is used as the input driver of the PA, a matching network is needed to ensure that the maximum amount of power from the source gets delivered to 


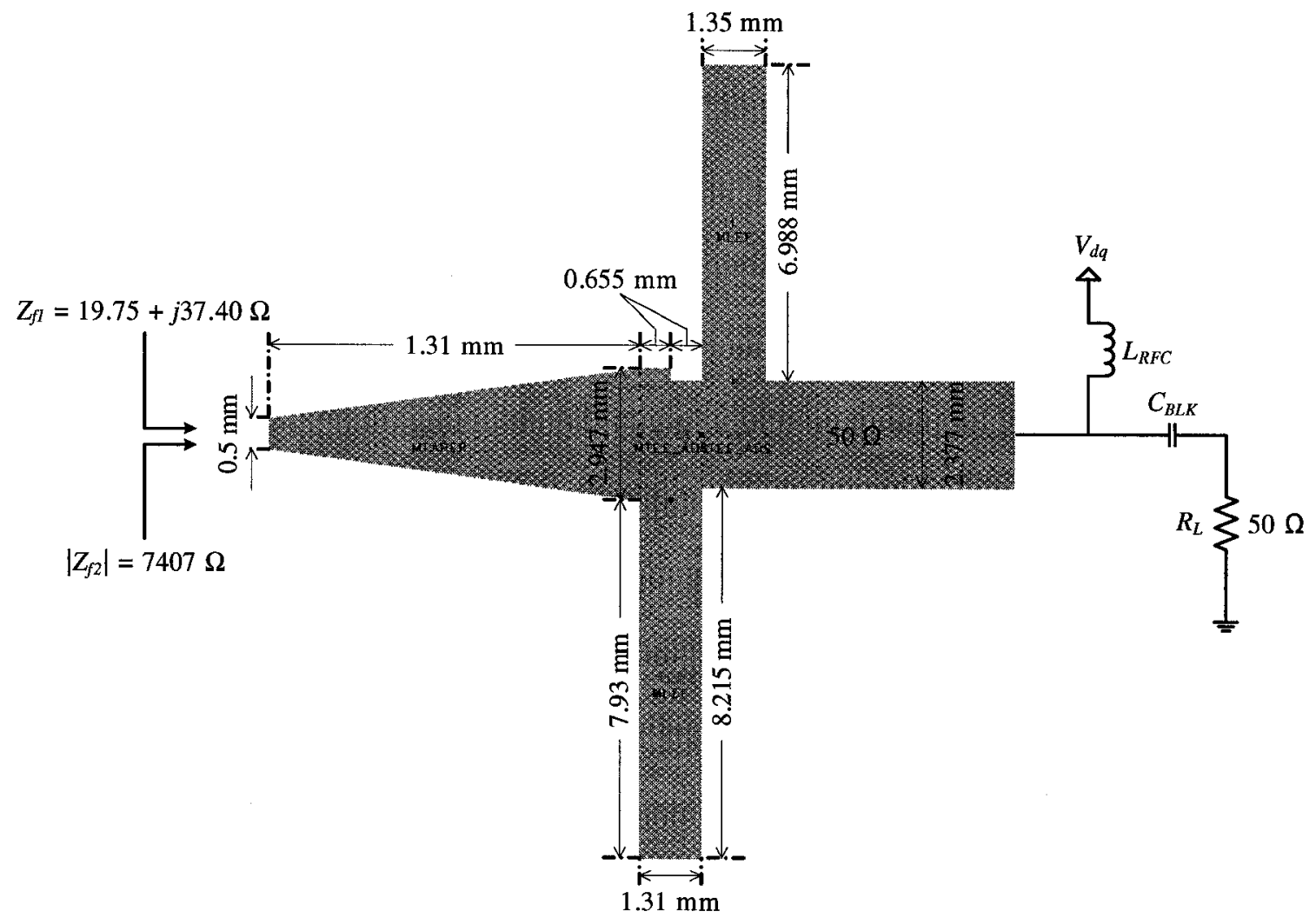

Figure 5.11: Microstrip transmission line layout of the Class E load network. 


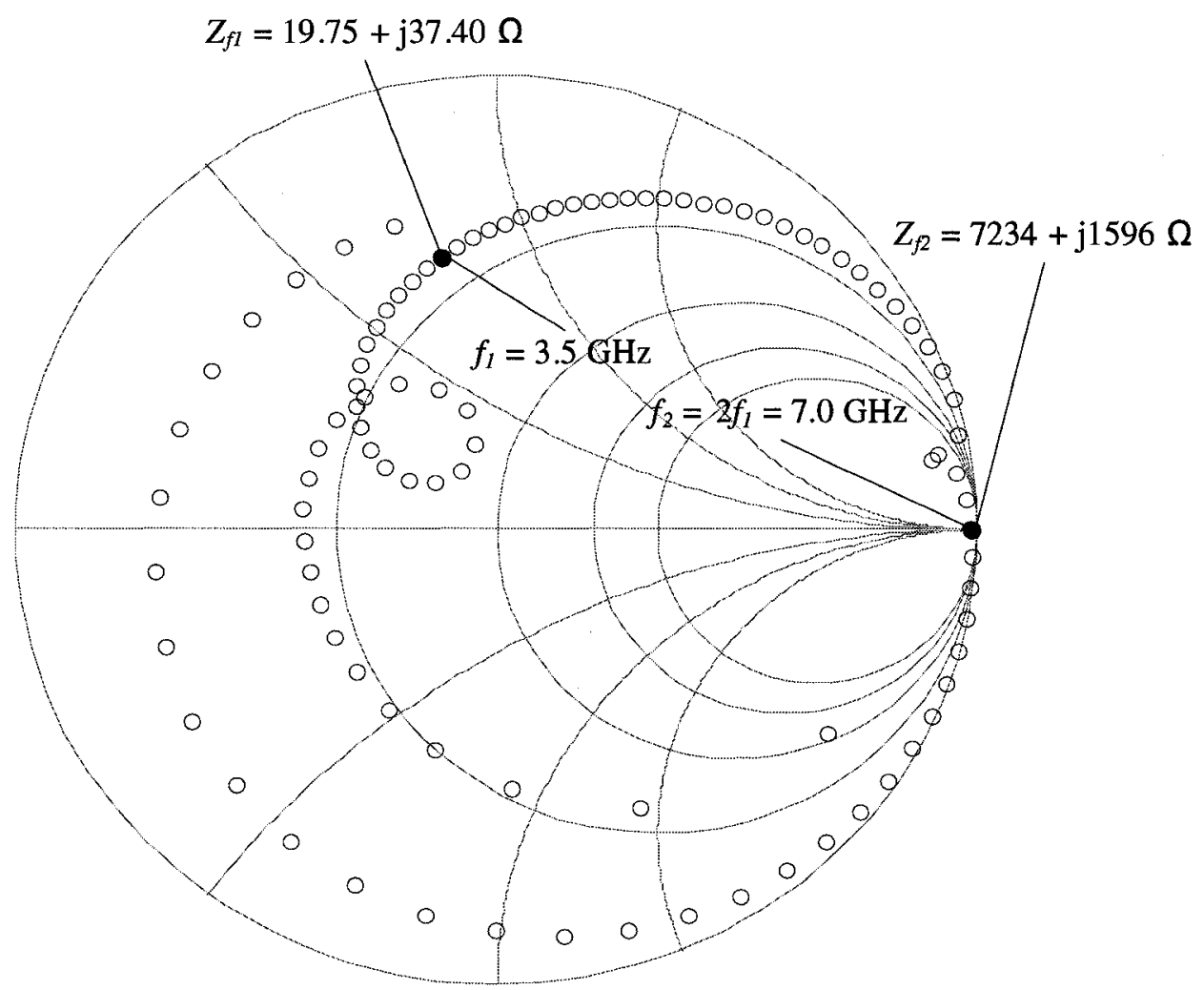

Figure 5.12: Simulated $S_{11}$ of the microstrip transmission line load network shows that the correct impedances for Class E operation are presented to output of the HEMT. 
the gate of the HEMT. The initial design strategy, referring to Figure 5.9, is to provide a conjugate, or small-signal input match to facilitate the characterization of the load network. The conjugate input match, shown only as an abstraction in Figure 5.9, is revealed in Figure 5.13 in its lumped-element form. The simulated $S_{11}$ response in Figure 5.14

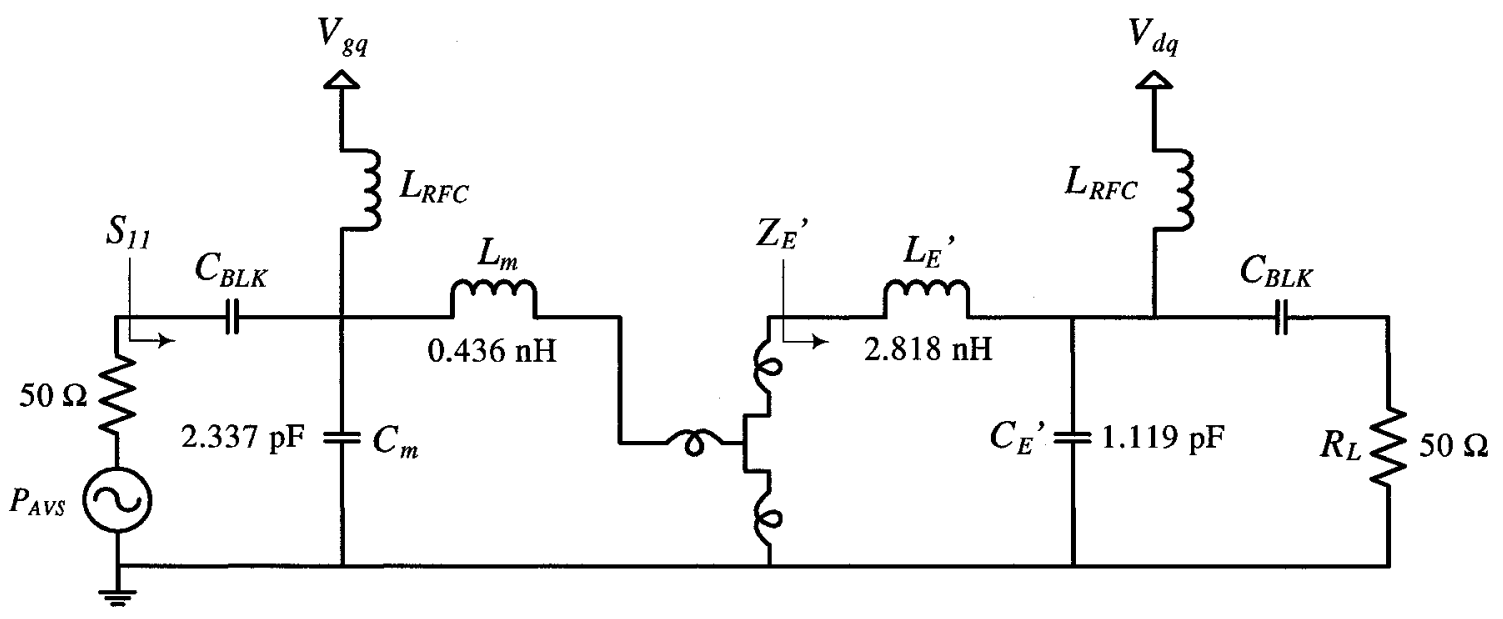

Figure 5.13: A conjugate input matching circuit is used to facilitate the Class E load network design. The small-signal input matching circuit is later replaced with an input matching circuit optimized for $25 \mathrm{dBm}$ of source power.

shows a return loss $(R L)$ of $40.037 \mathrm{~dB}$. This is the circuit's response to a small-signal excitation which captures only the approximate behaviour of a nonlinear PA.

The drive level at the gate must be sufficiently high to switch the HEMT $\left(P_{A V S}=25\right.$ $\mathrm{dBm}$ ). Applying this level of RF power to a matched $50 \Omega$ input, corresponds to a 11.247 $\mathrm{V}$ peak-to-peak voltage swing at the gate terminal, taking the transistor outside of the small-signal regime. A power match at the input with fixed Class E loading at the output (see Figure 5.11) is shown here in the final phases of the design process as a means to obtain the best possible performance from the PA. The input matching circuit design proceeds as follows. 


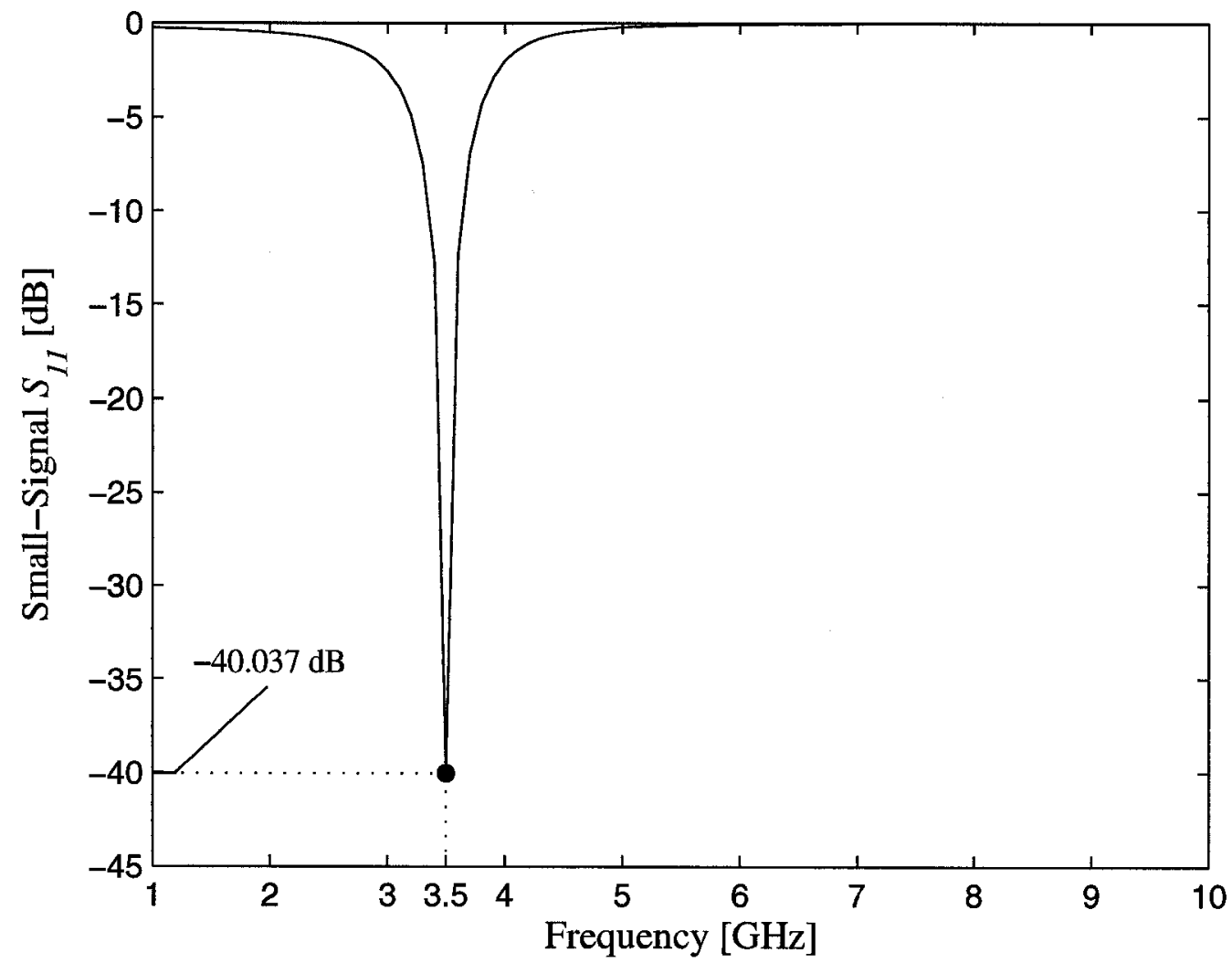

Figure 5.14: Simulated $S_{11}$ of the Class E PA with a conjugate input matching circuit. 
1. The gate of the HEMT must be driven by the required RF power level: $P_{A V S}=$ $P_{S W}=25 \mathrm{dBm}$.

2. The input matching circuit must be tuned until drain efficiency and output power are maximized for the applied input power level in (1) and fixed output loading as shown in Figure 5.13.

Performing a large-signal match at the input of the PA for $P_{A V S}=25 \mathrm{dBm}$ yields the circuit in Figure 5.15 and the corresponding large-signal $S_{11}$ response in Figure 5.16. Simulating this circuit in the small-signal domain gives the PA's small-signal input re-

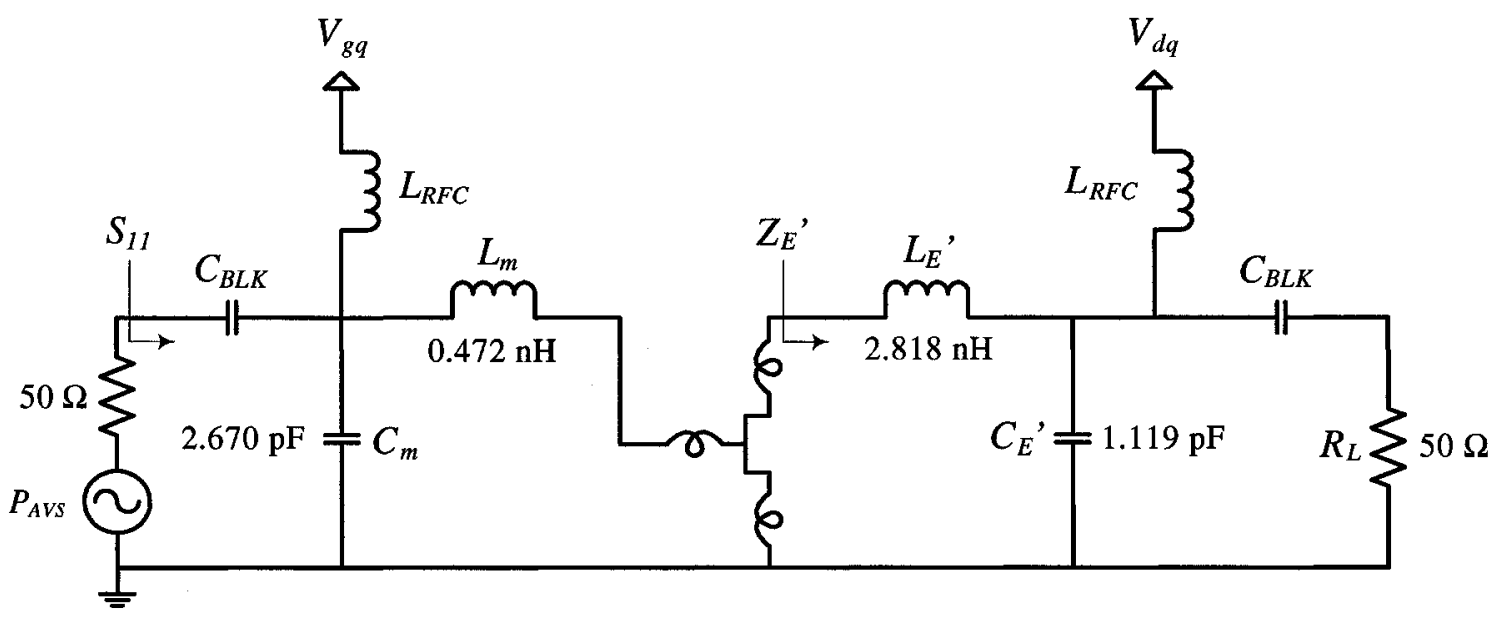

Figure 5.15: Class E PA with the input of the device power matched to the $50 \Omega$ source impedance.

sponse, which is also shown in Figure 5.16, to illustrate the importance of large-signal impedance matching. The difference in the small- and large-signal impedances surfaces when the performance of the PA is assessed. Table 5.2 gives a brief comparison of how the circuit performs with a conjugate matching circuit versus a power match at a source power of $25 \mathrm{dBm}$. All parameters are slightly higher with the power match at the input; 


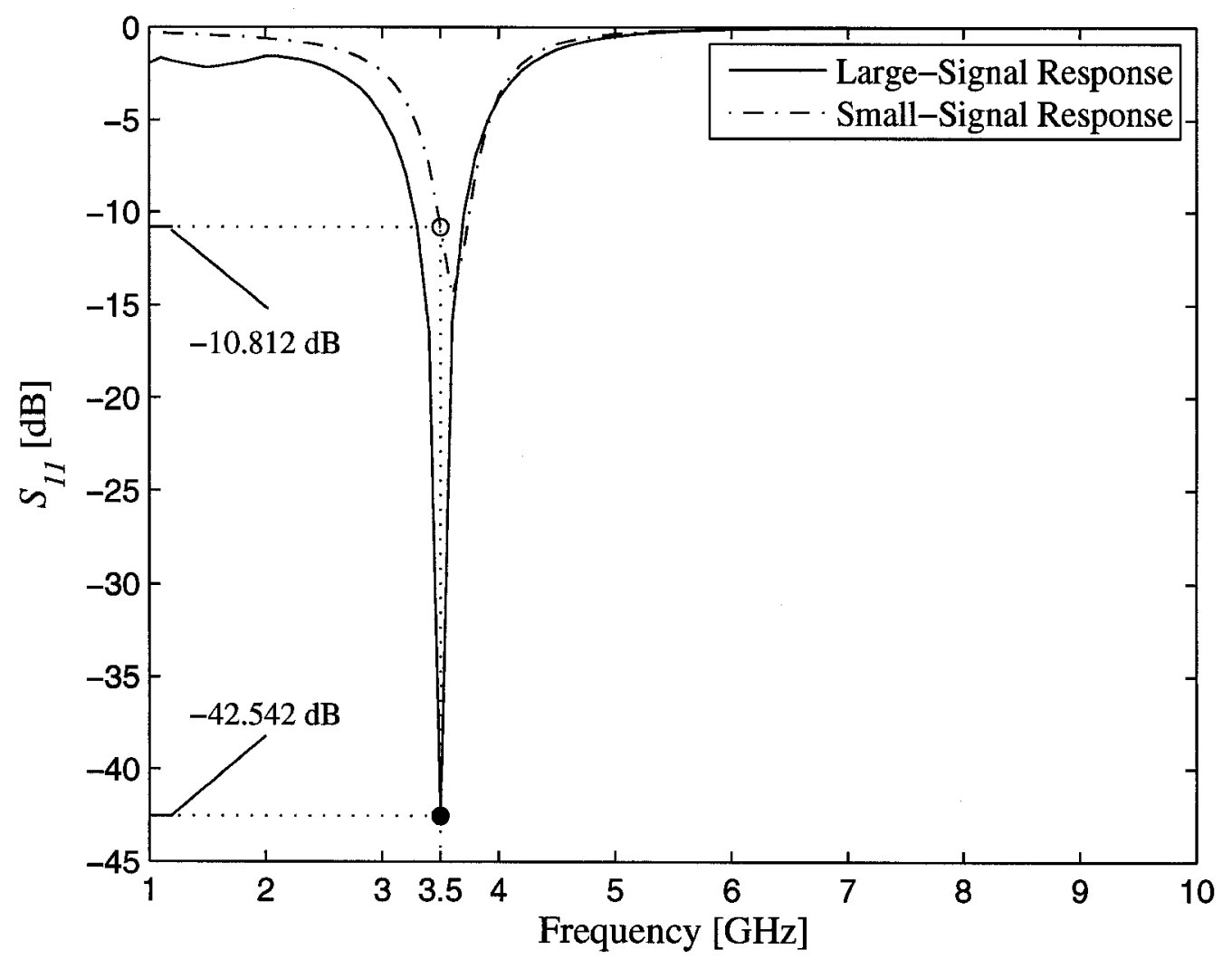

Figure 5.16: Simulated large-signal $S_{11}$ of the Class E PA with the input of the device power matched to $50 \Omega$ at $25 \mathrm{dBm}$ of source power. Also shown is the $S_{11}$ response of the circuit in Figure 5.15 simulated under small-signal conditions.

\begin{tabular}{|c|c|c|}
\hline Parameter & Small-Signal Input Match & Large-Signal Input Match \\
\hline \hline$P_{\text {out }}[\mathrm{dBm}]$ & 31.723 & 31.969 \\
\hline$G_{T}[\mathrm{~dB}]$ & 6.723 & 6.969 \\
\hline$D E[\%]$ & 65.005 & 66.891 \\
\hline$P A E[\%]$ & 51.182 & 53.448 \\
\hline
\end{tabular}

Table 5.2: Class E PA performance for small- and large-signal input matching circuits. 
two conclusions can therefore be made:

1. The Class E PA operates more efficiently and with higher output power when the gate of the HEMT is loaded with a large-signal matching circuit than when loaded with a conjugate matching circuit

2. The improvement with a power input match is significant, but not drastic. The design strategy of using a conjugate input match to evaluate the performance of the load network and to estimate the overall performance of the PA is therefore valid.

The lumped-element input matching circuit gives a high large-signal return loss of $42.542 \mathrm{~dB}$, making it suitable for synthesis into a microstrip equivalent circuit as shown in Figure 5.17.

\subsection{Bias Insertion Network and Stability}

Considerable effort is always bestowed upon power amplifier matching circuitry so that typical power amplifier specifications, such as gain, output power, and efficiency are met. Comparatively speaking, the manner in which bias is inserted into the PA and the effects of the bias insertion circuit on the power amplifier is usually the least considered facet of the overall design [78].

The input and output bias circuits in this work are considered integral parts of the Class E microwave power amplifier and so are included in every part of the design process. They are designed to interface with a $50 \Omega$ system and to not interfere with the PA's input and output matching circuitry. The impedances are set up as shown in Figure 5.18. The 


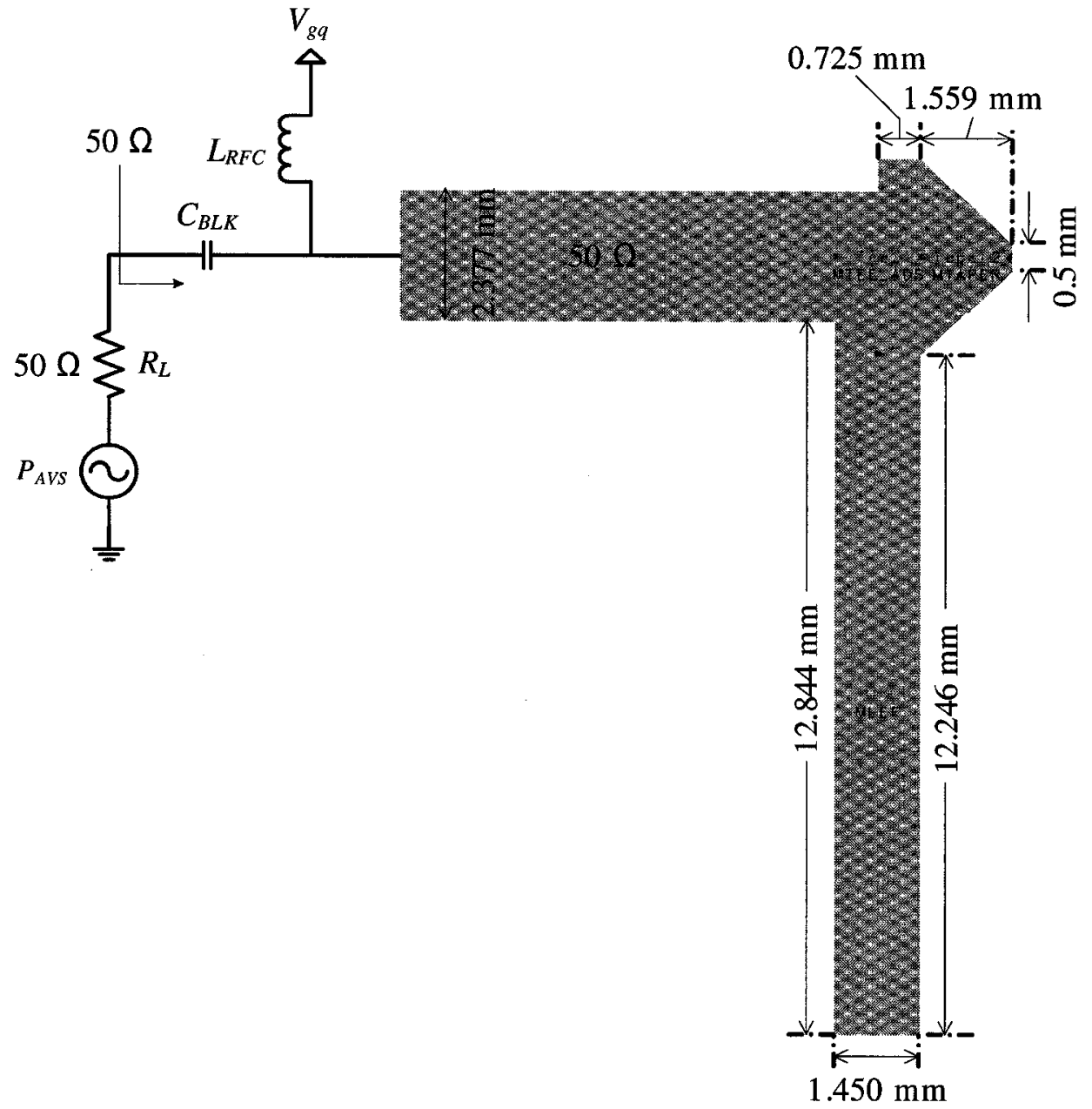

Figure 5.17: Layout and dimensions of input matching circuit for Class E PA. 


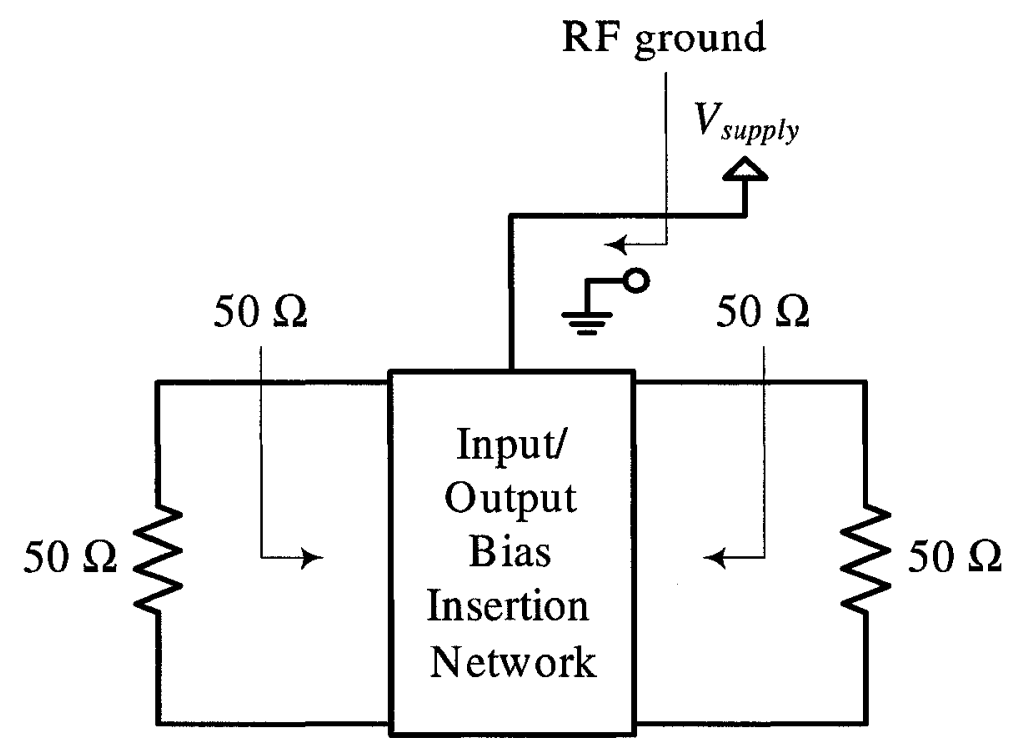

Figure 5.18: System-level schematic of input and output bias insertion circuits in a $50 \Omega$ system.

bias insertion circuits at the input and output of the transistor are essentially the same, with differences only in the stabilizing elements and in the tolerances of the components used. The key requirements of the bias circuit are as follows.

- A stable operating point must be presented to the transistor in spite of the large signal variations at the input and output ports of the transistor.

- The power supply should ideally be decoupled over the entire frequency range over which the HEMT has current gain (up to $f_{T}$ ).

- The point at which the bias circuit interfaces with the PA's matching circuitry should be an open-circuit over the RF band of interest ( $3.5 \mathrm{GHz}$ to at least $7 \mathrm{GHz}$ )

- The desired RF signal at 3.5 GHz must be properly AC-coupled in to and out of the PA. 
- Out-of-band oscillation suppression should be included.

- Protection circuitry is required to prevent transistor from catastrophic burn-out during the power-up sequence.

A system-level schematic for the design that follows is shown in Figure 5.19; the blocks in the schematic are dissected in a four-part series. The first part deals with power

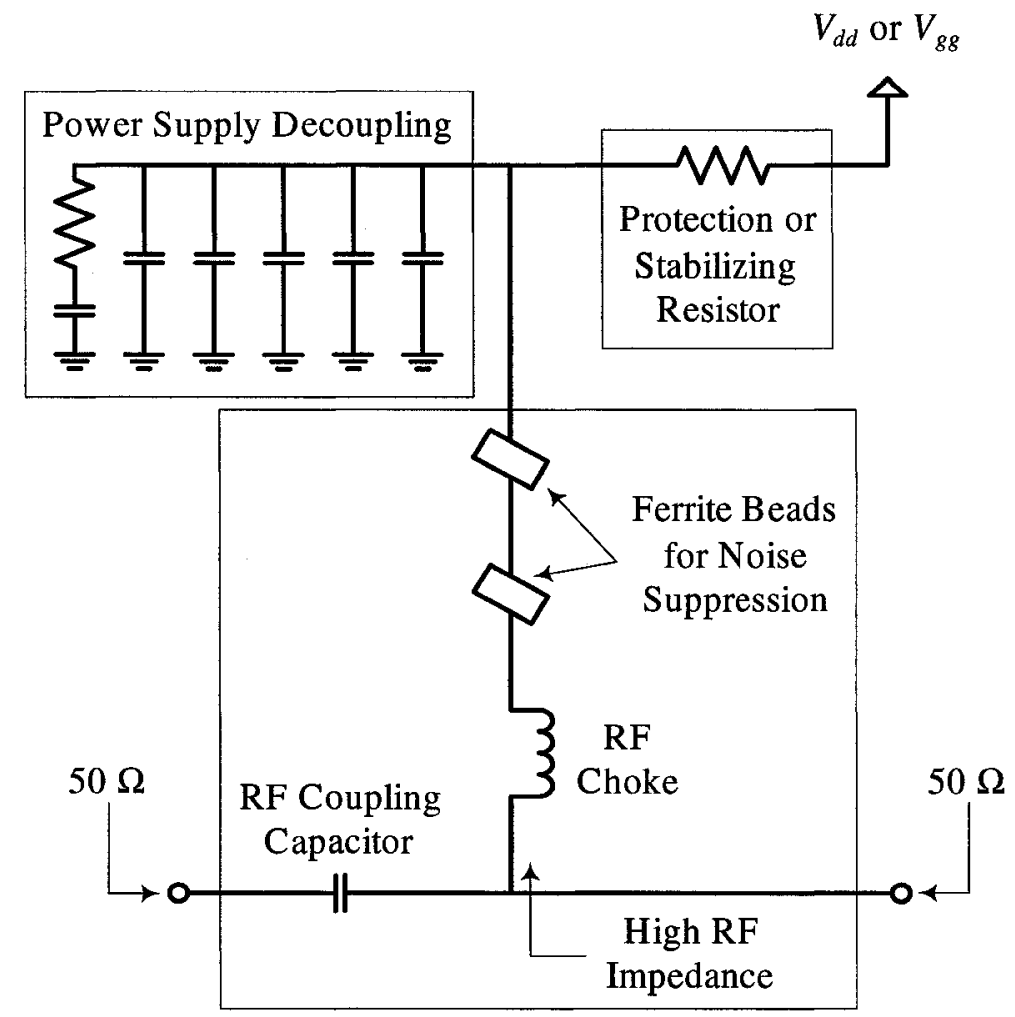

Figure 5.19: Input and output bias insertion circuits (in a $50 \Omega$ system), consisting of power supply decoupling, protection, and noise suppression circuitry, provide a stable operating point to the Class E PA.

supply decoupling. This is followed by a discussion on in-band RF loading and out-ofband oscillation suppression. Stability is a common theme throughout the design and is touched upon in all sections. In the final PA design however, small-signal stability is explicitly enforced with a series resistance in the input bias feed. Incidently, the same 
technique is used to prevent transistor burn-out. It is only appropriate then, to discuss protection and stability here.

\subsubsection{Power Supply Decoupling}

The power supply decoupling circuit plays a major role in eliminating power supply ripple that could otherwise couple into the gate of the HEMT via the intrinsic feedback capacitance between the drain and the gate. Ideally, one designs the decoupling network to present an $\mathrm{RF}$ short-circuit to the power-supply leads from the $\mathrm{MHz}$ range up to the HEMT's $f_{T}$. Such a broadband low-impedance path to ground is acheived with a bank of parallel capacitors connected as close as possible to the power supply connection of the PA.

The power supply lead itself presents a significant source of inductance. Any sudden change in current, for example when the power supply is turned on, causes the lead inductance to respond with a corresponding voltage spike, which could cause low-frequency instability in the PA circuit. A large electrolytic capacitor is placed closest to the power supply lead in order to shunt low-frequency noise. Higher capacitance values are placed in parallel for any high-frequency ripple that may be present in the supply path. Large separation between capacitance values is chosen to prevent unwanted resonances; a de-Q'ing resistor is used where large capacitance separation is not possible. Figures 5.20 and 5.21 show the decoupling circuit and the corresponding simulation. A reasonable short circuit is maintained up to at least the second harmonic at $7 \mathrm{GHz}$. Unfortunately, component data does not allow simulation up to the HEMT's $f_{T}$ of $13 \mathrm{GHz}$. 


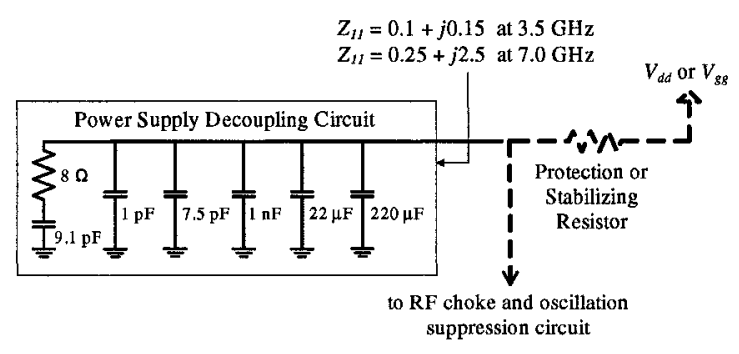

Figure 5.20: The power supply decoupling circuit provides a broadband RF short-circuit to the power supply.

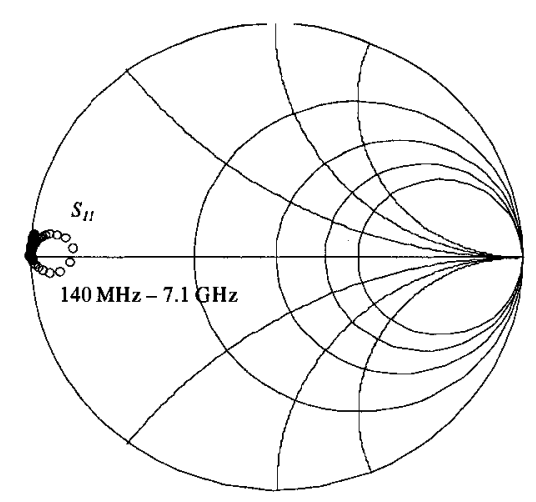

Figure 5.21: $S_{11}$ response of the power supply decoupling circuit shows that a short circuit is maintained up to at least $7 \mathrm{GHz}$.

\subsubsection{RF Loading and Oscillation Suppression}

Special attention is paid to the drain bias network, more so than the gate bias, since it is the loading at the output that primarily determines the performance of the PA. Once the output bias network is designed, it is simply duplicated for the input with some minor adjustments.

It is common practice to choose an $\mathrm{RF}$ choke inductance value whose reactance is at least a factor of ten greater than the drain impedance [79]. The measured drain impedance of the GaN HEMT is $14.2 \Omega$, and so a large inductance of $12 \mathrm{nH}$ is selected whose self-resonance frequency is at $6.7 \mathrm{GHz}$ and average current handling capability is $800 \mathrm{~mA}$.

Most large devices exhibit potential instability at low frequencies where the port impedances are defined primarily by the bias insertion networks [32]. In addition to the noise suppression offered by the decoupling circuit, ferrite beads are placed in series with the RF choke to provide a high resistance path for frequencies from $3 \mathrm{GHz}$ down to 
$10 \mathrm{MHz}$. The performance of a ferrite bead is similar to that of an inductor, except the high RF impedance offered by the ferrite is primarily resistive, instead of reactive. The series configuration of the $12 \mathrm{nH}$ inductor and the ferrite beads is shown in Figure 5.22. The simulation from $10 \mathrm{MHz}$ to $3 \mathrm{GHz}$ (upper frequency limited by simulation data) shows that high lossy impedance is maintained up to at least $3 \mathrm{GHz}$ (see Figure 5.23); the reactance of the inductor dominates the response up to $7 \mathrm{GHz}$. In addition to sup-

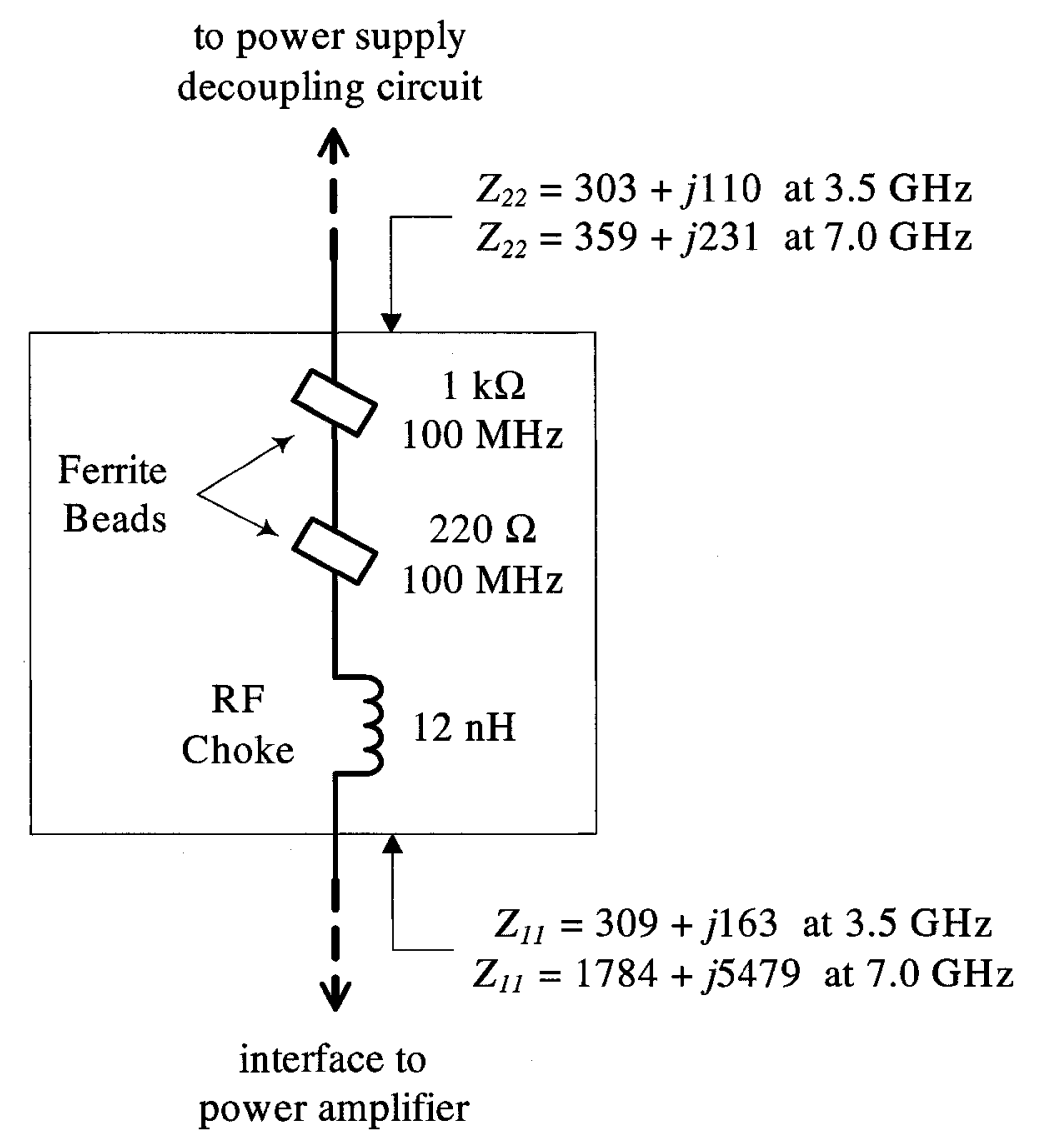

Figure 5.22: RF choke and ferrite beads offer a stable operating point to the PA without interfering with matching circuitry.

pressing any frequency components that get past the capacitor bank, the ferrite beads give the circuit the added benefit of suppressing potentially harmful oscillations that may be caused by ringing in the large inducance. 


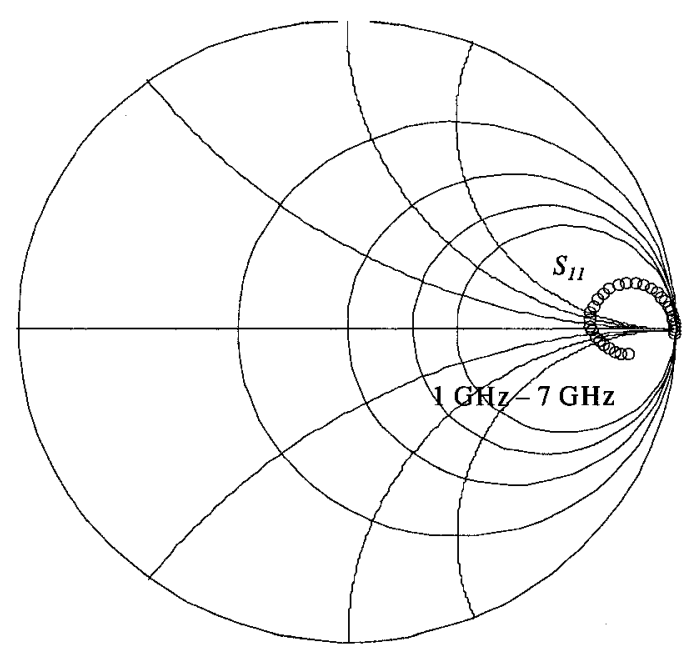

(a)

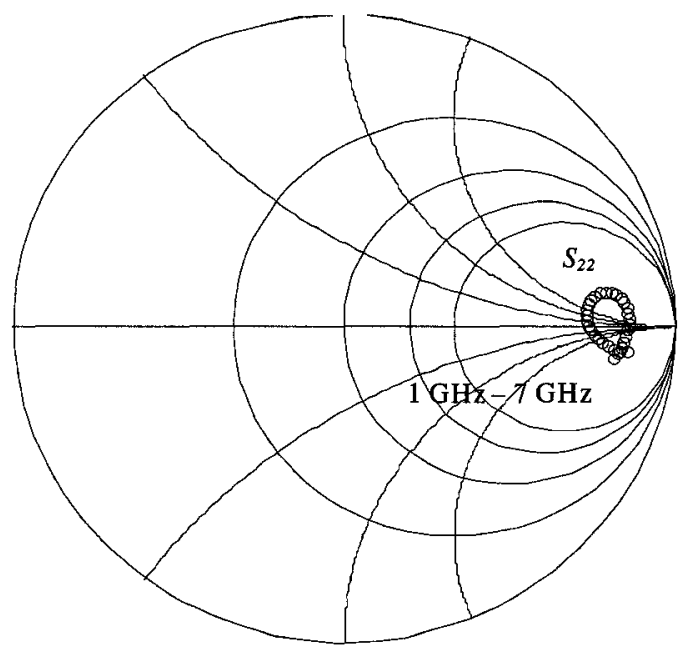

(b)

Figure 5.23: (a) $S_{11}$ and (b) $S_{22}$ responses of the noise suppression/bias feed circuit shows high (primarily resistive) impedances looking into both ports of the circuit in a $50 \Omega$ system.

The bias network design, by virtue of the properly decoupled power supply and high RF impedance presented up to $7 \mathrm{GHz}$, does not load the matching circuitry of the PA. To guarantee that the DC bias does not interfere with the signal source at the input or the measurement equipment at the output, the input and output signals are coupled to the circuit with $7.5 \mathrm{pF}$ coupling capacitors whose self-resonance frequency coincides with the $3.5 \mathrm{GHz}$ fundamental tone. The input and output ports of the bias network are effectively transparent to each other at $3.5 \mathrm{GHz}$.

\subsubsection{Protection and Stability}

As an added measure of protection to the transistor, a $33 \Omega$ resistance is added to the power supply lead at the drain bias port. This is done to ensure that any stored charge at the supply terminals does not dissipate entirely through the low impedance drain of the HEMT, as this may cause device failure. 
The same technique is used at the input bias port; a $620 \Omega$ resistor is placed in series with the input bias terminal for stability in addition to transistor protection.

\subsubsection{Complete Circuit Simulation}

The power supply decoupling circuit, protection resistor, and bias feed are simulated together with all pad metallization to account for transmission line effects. The PA is designed with $50 \Omega$ input and output terminations. The simulations in Figure 5.24 show that the total circuit (see Figure 5.19) is suitable for integration into a $50 \Omega$ system.

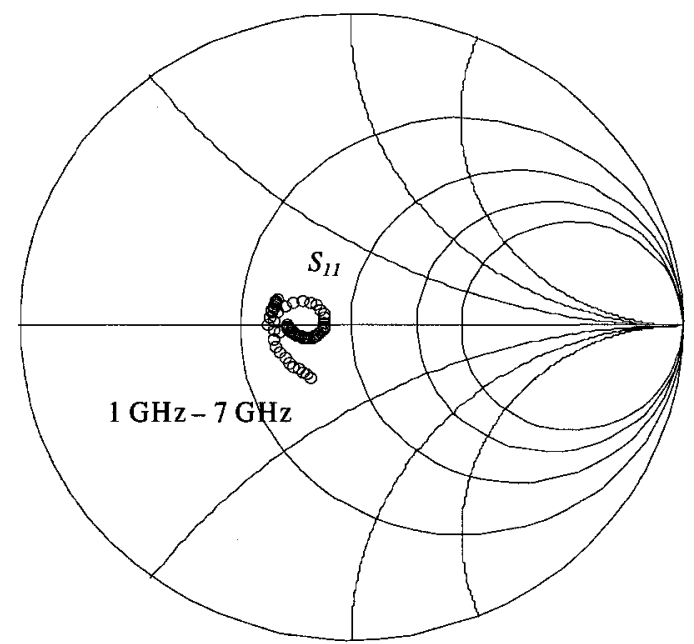

(a)

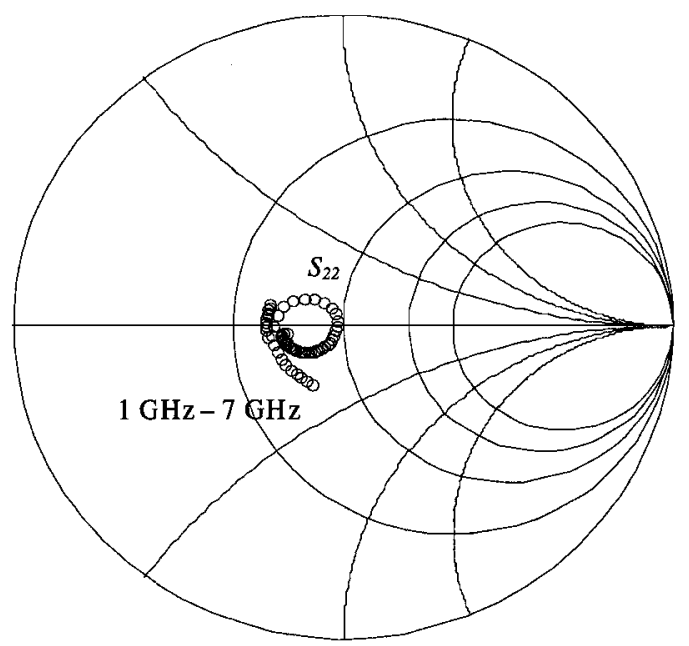

(b)

Figure 5.24: (a) $S_{11}$ and (b) $S_{22}$ responses of the complete bias insertion circuit show input and output impedances close to $50 \Omega$.

With the bias insertion circuits integrated into the PA module, small-signal stability is checked. The Rollet stability factor (or $k$-factor) simulation is shown in Figure 5.25. The simulation shows that the PA is unconditionally stable (since $k>1$ ) for small signal excitation. 


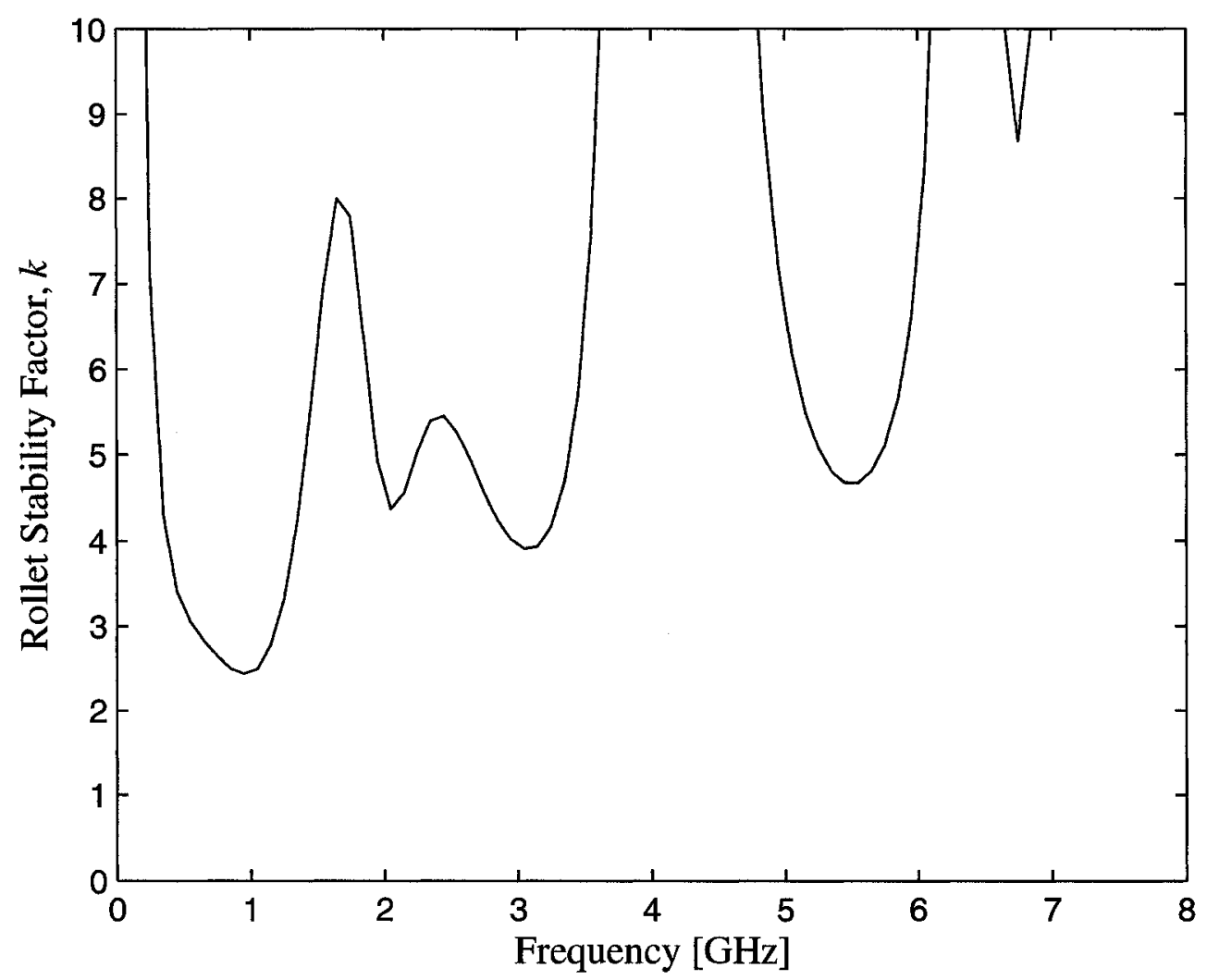

Figure 5.25: Small-signal stability simulation shows that the power amplifier, including all metallization and bias circuitry, is unconditionally stable (since $k>1$ ) from $50 \mathrm{MHz}$ up to $7.1 \mathrm{GHz}$. Note that large signal stability is verified during testing. 


\subsection{Thermal Considerations}

The hardware required for heat removal in base station power transmitters represents a significant investment in the overall cost of a PA. The large amount of power that is dissipated as heat in the device requires a good thermal ground for efficient PA operation.

Even though the Class E PA is highly efficient, an aluminum base plate is designed and manufactured, as a precautionary measure, to pull heat away from the device. The design is shown in Figure 5.26. The key feature is the integration of a raised pedestal to allow the HEMT die to be directly attached to the heat sink with thermally conductive epoxy.

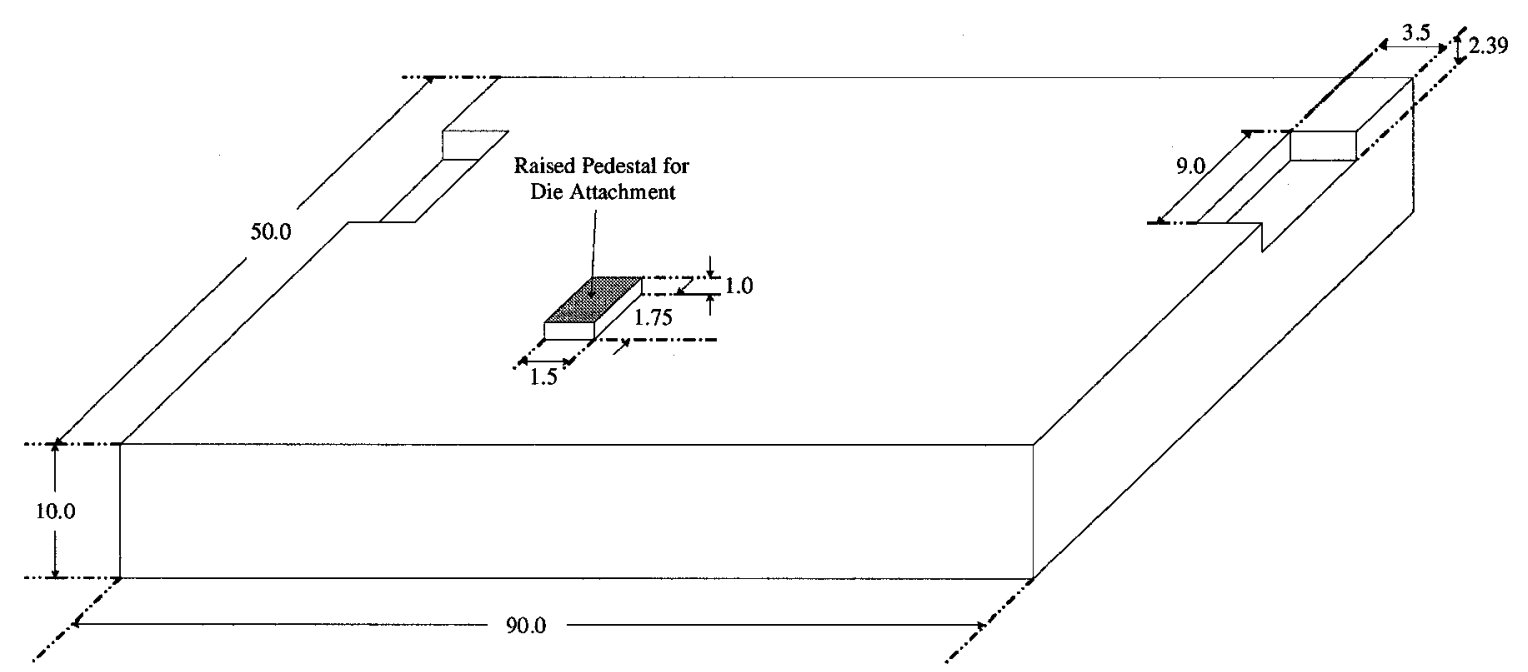

Figure 5.26: Custom aluminum base plate design. The base plate functions to provide mechanical support to the PCB and to pull heat away from the HEMT.

\subsection{Simulated and Measured Results}

The 3.5 GHz Class E GaN-on-Si power amplifier is shown fully assembled in Figure 5.27. Measured performance is compared with simulations wherever possible. The PA performs 


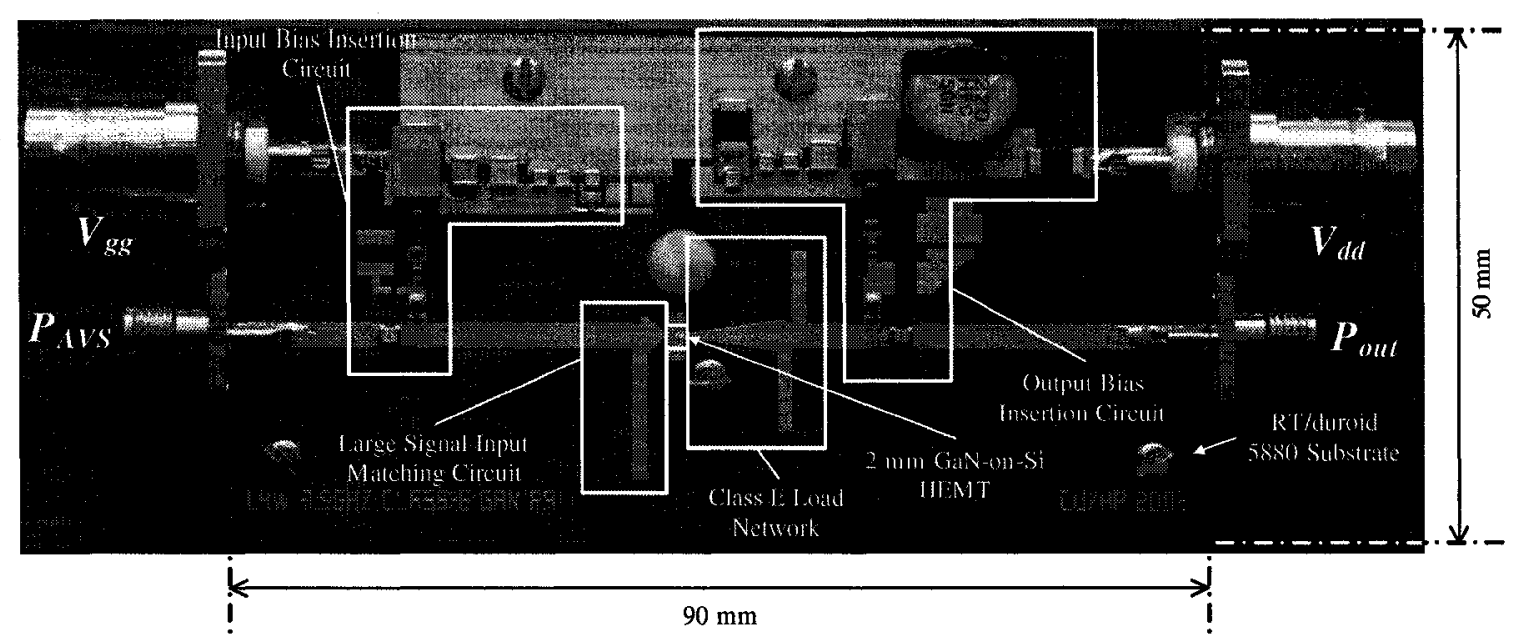

Figure 5.27: Fully assembled 3.5 GHz GaN-on-Si Class E power amplifier.

better than expected when tested over the standard performance metrics. Measured results correlate with simulated performance in that the same operation is observed with a relatively constant offset in input power level.

The RF test set-ups for both small-signal and power measurements are shown in Figure 5.28. The difference between the two is that a vector network analyzer (VNA) cannot be used for large-signal power measurements due to the power levels required. The PA module is tested by first evaluating the quality of the input match with the

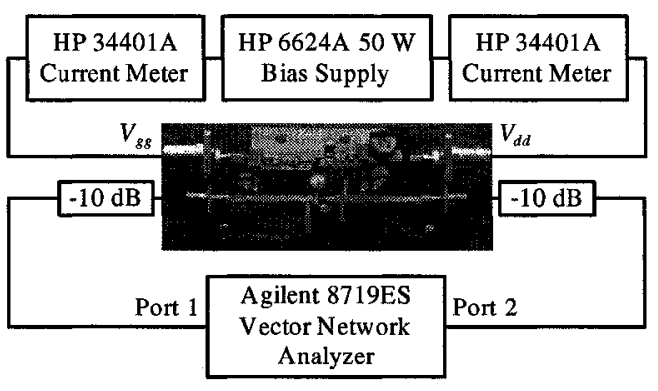

Testbench for SmaltSignal Measurements

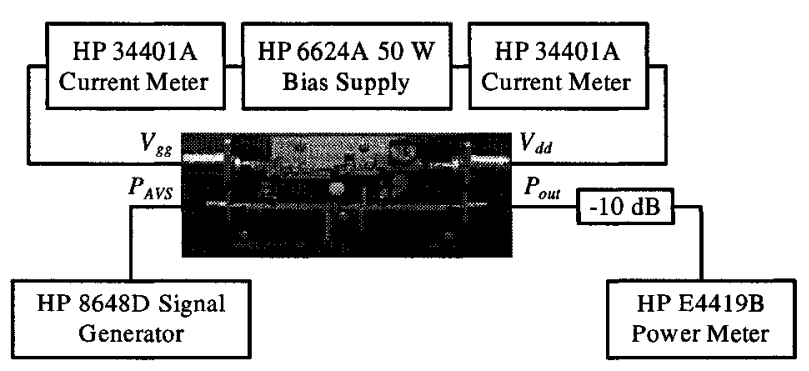

Testbench for Power Measurements

Figure 5.28: Lab set-ups for both small-signal and power measurements of the Class E PA shown in Figure 5.27.

small-signal testbench. A full suite of power measurements is done using the standard 
microwave testing equipment shown in Figure 5.28. The power amplifier is designed to operate with an input power level of $25 \mathrm{dBm}$. This power level is considered to border the unsafe region of operation in this GaN HEMT process. A conservative approach is therefore taken in the measurement process to avoid catastrophic device failure.

Accurate characterization of the input match to this PA requires a large-signal network analyzer. Such microwave test equipment is non-standard, expensive, and as is the case here, not available. A VNA is commonly used instead to provide a reasonable estimate of the quality of input match. The agreement between the measured and simulated $S_{11}$ is seen in Figure 5.29. It is interesting to note that the small-signal input return loss, in

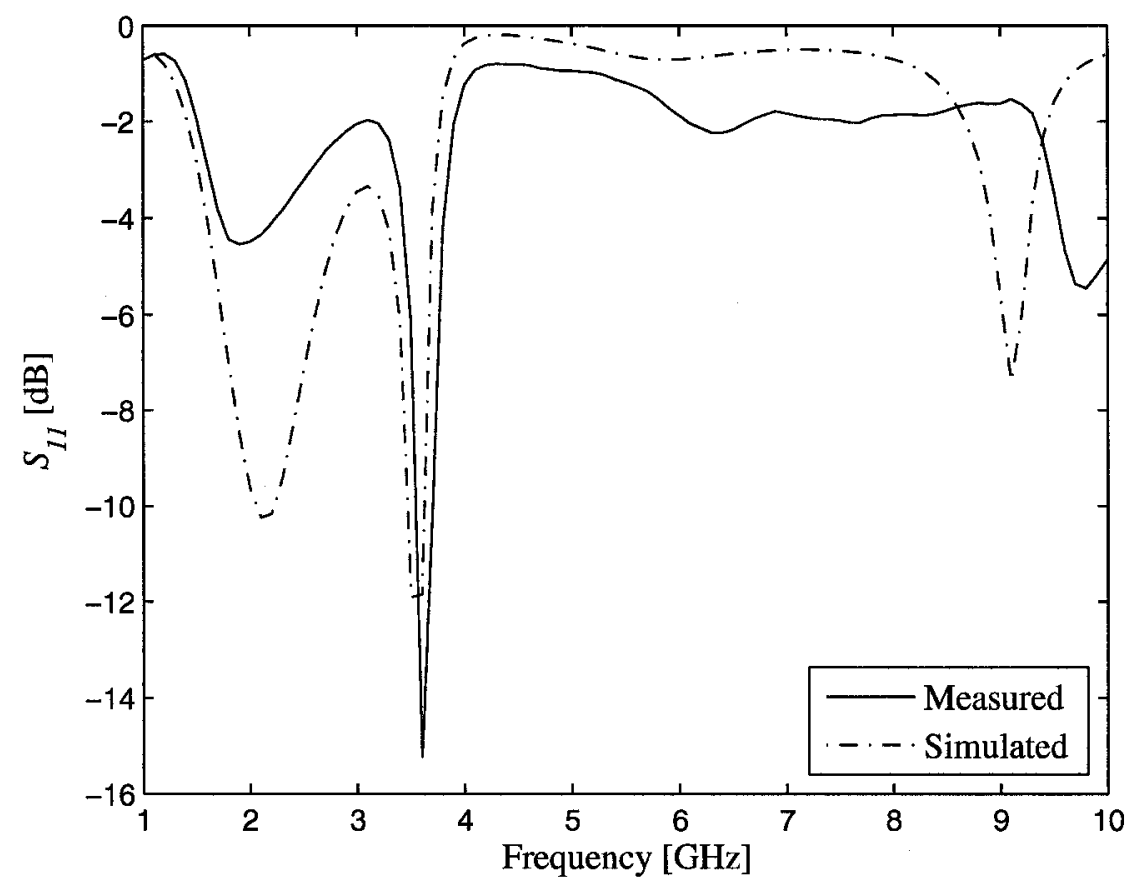

Figure 5.29: Measured and simulated $S_{11}$ responses.

both the measured and simulated responses, is maximized at $3.6 \mathrm{GHz}$, where measured and simulated $R L$ is $15.234 \mathrm{~dB}$ and $11.845 \mathrm{~dB}$, respectively. At $3.5 \mathrm{GHz}$, the input 
return loss is $11.9 \mathrm{~dB}$ (simulated) and $6.1 \mathrm{~dB}$ (measured). The best power performance however is seen at the design frequency of $3.5 \mathrm{GHz}$, thereby validating the large-signal input matching approach used in the design process. Figure 5.30 shows measured gain and power-added efficiency as functions of input frequency from $3.5 \mathrm{GHz}$ to $3.7 \mathrm{GHz}$. Although no data below $3.5 \mathrm{GHz}$ are shown, gain was seen to decrease in a similar fashion below the design frequency.

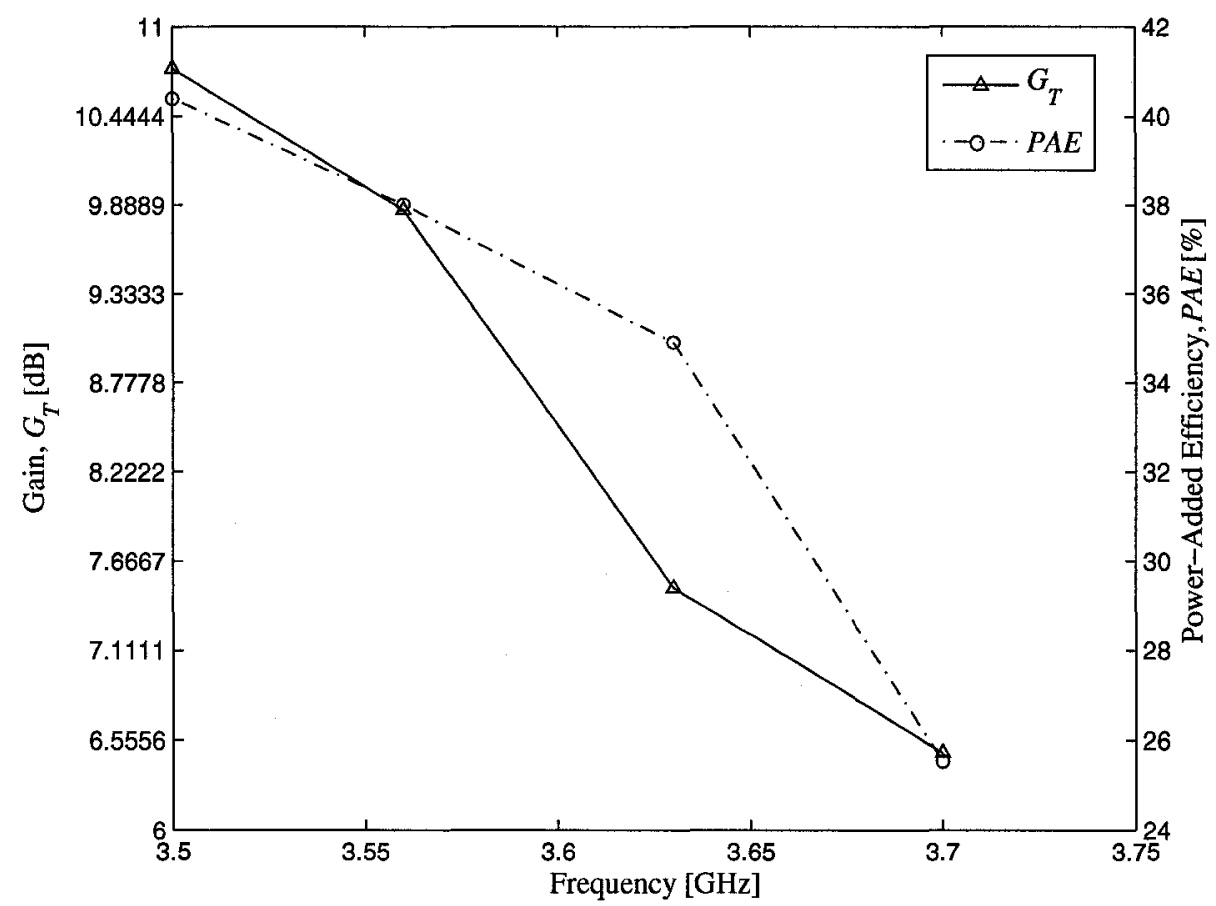

Figure 5.30: The measured $G_{T}$ and $P A E$ plot shows that PA performance is best at the design frequency of $3.5 \mathrm{GHz}$.

In an ideal test set-up, Class E operation would be best verified by sampling the drain voltage waveform while operating the PA at the optimally designed input power level (25 dBm). Regrettably, such high-frequency voltage probing equipment is not readily available, so only simulated drain voltages and currents can be shown. The drain voltage 
and current waveforms in Figure 5.31 confirm that, at least in simulation, the PA operates in quasi Class $\mathrm{E}$ mode. The simulations agree with previously reported work noting that the desired (ideal) peak voltage and current levels of $71 \mathrm{~V}$ and $660 \mathrm{~mA}$ are never reached in practice [76].

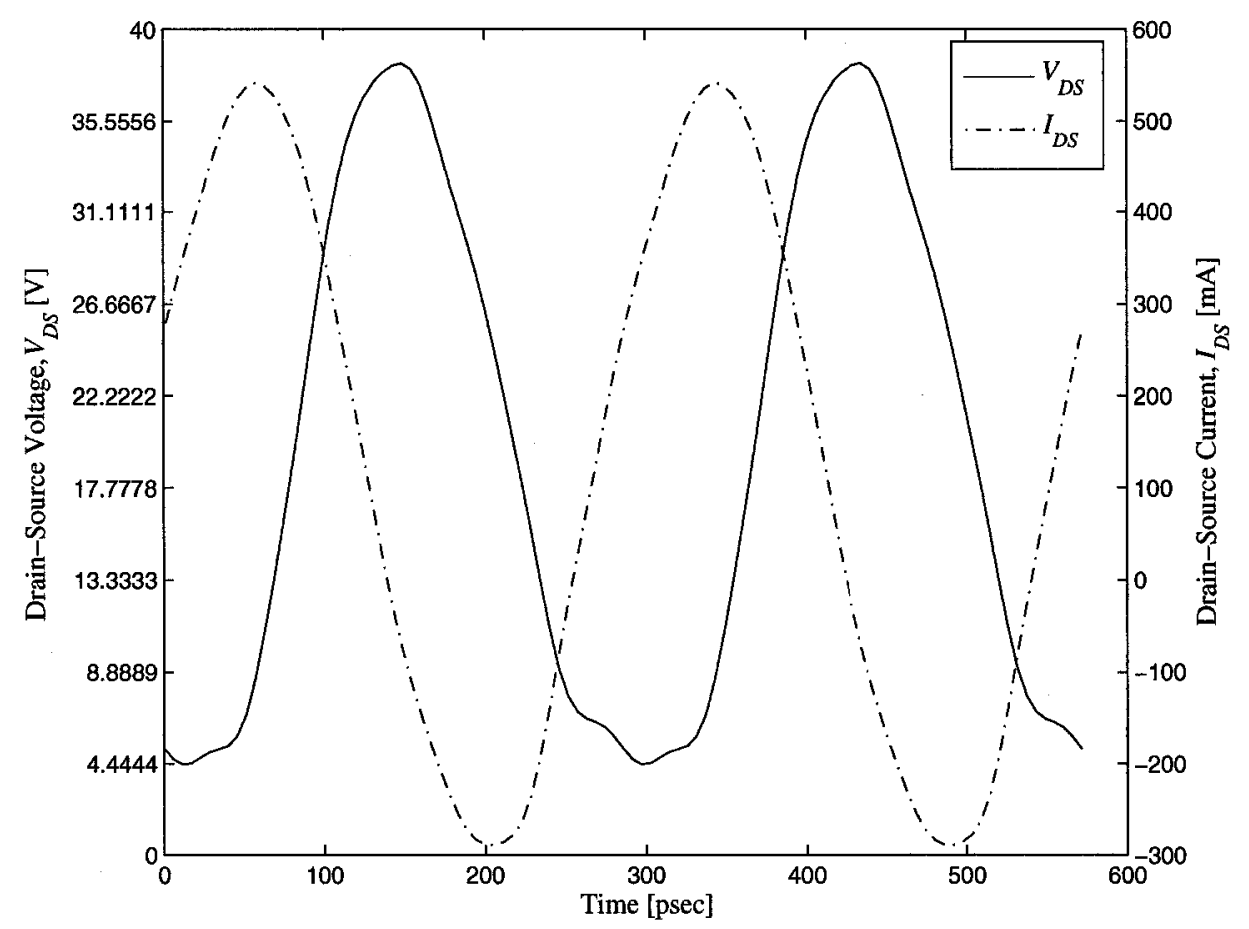

Figure 5.31: Simulation of the Class E voltage and current waveforms for the complete microstrip transmission line Class E PA.

The true test of a PA's capability to amplify a desired RF input signal lies in its ability to do so as efficiently as possible with a reasonable gain. The PA demonstrated here is intended to be the final gain stage immediately before the transmitting antenna. The large power level delivered by the final PA stage requires maximum efficiency since most of the gain is provided be the preceeding stages in the system. This is not to say that gain is not an important consideration in the design of a high efficiency PA. The plots 
in Figure 5.32 compare the PA's measured output response with simulations. It is clear
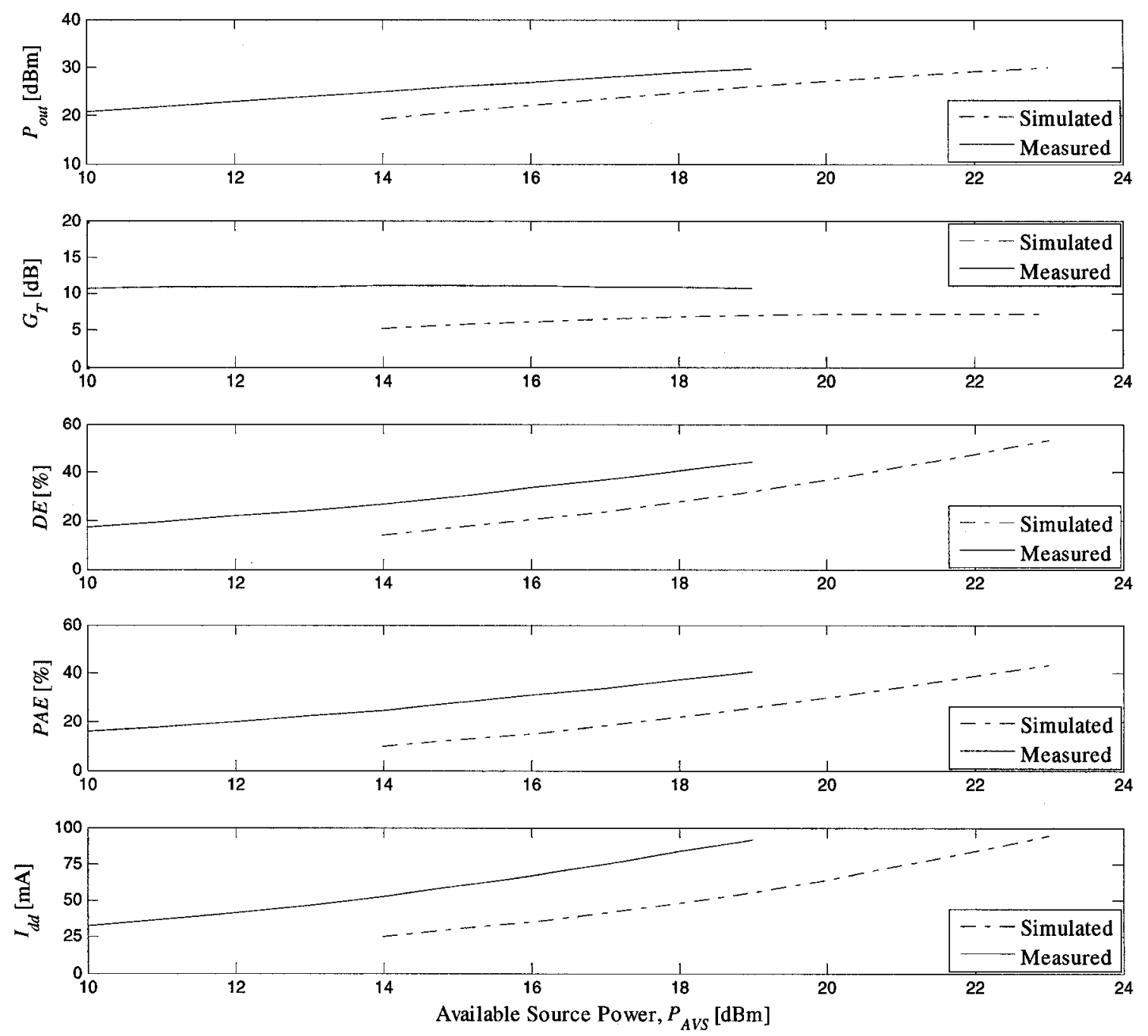

Figure 5.32: The GaN Class E PA performs better than expected. There is a uniform 3.5 $\mathrm{dB}$ input power offset between measured and simulated results.

that the PA performs better in practice than in simulation. Most notable is the large difference in gain $\left(G_{T}\right)$ : at $P_{A V S}=19 \mathrm{dBm}$, measurements show a $G_{T}$ of $10.74 \mathrm{dBm}$, while simulations predict $G_{T}$ to be only $6.97 \mathrm{~dB}$. Fundamental output power $\left(P_{\text {out }}\right)$, drain efficiency $(D E)$, power-added efficiency $(P A E)$, and drain current $\left(I_{D S}\right)$ all reflect this larger gain by exhibiting results almost identical to simulated values, except at a uniformly 
lower $P_{A V S}$. The $3.5 \mathrm{~dB} P_{A V S}$ offset in measured versus simulated results is attributed to the GaN HEMT in the PA module having a transconductance $\left(g_{m}\right)$ that is higher than that predicted by the device model. All discrepancies aside, the power amplifier performs very well; Table 5.3 summarizes measured versus simulated testing conditions and overall performance of the PA at $3.5 \mathrm{GHz}$ with $P_{A V S}=19 \mathrm{dBm}$.

\begin{tabular}{|c|c|c|c|}
\hline & \multirow{2}{*}{ Parameter } & \multicolumn{2}{|c|}{ Simulated } \\
\cline { 3 - 4 } & Measured & $P_{A V S}=22.5 \mathrm{dBm}$ & $P_{A V S}=19 \mathrm{dBm}$ \\
\hline \hline Drain bias supply voltage $\left(V_{d d}\right)$ & $23.4 \mathrm{~V}$ & $23.8 \mathrm{~V}$ & $23.8 \mathrm{~V}$ \\
Drain bias supply current $\left(I_{d d}\right)$ & $91.2 \mathrm{~mA}$ & $89.2 \mathrm{~mA}$ & $51.4 \mathrm{~mA}$ \\
Gate bias supply voltage $\left(V_{g g}\right)$ & $-2.95 \mathrm{~V}$ & $-3.1 \mathrm{~V}$ & $-3.1 \mathrm{~V}$ \\
Gate bias supply current $\left(I_{g g}\right)$ & $3.1 \mu \mathrm{A}$ & $0 \mathrm{~A}$ & $0 \mathrm{~A}$ \\
\hline \hline Input return loss $(R L)$ & $6.1 \mathrm{~dB}$ & $11.8 \mathrm{~dB}$ & $11.8 \mathrm{~dB}$ \\
3.5 GHz output power $\left(P_{\text {out }}\right)$ & $29.7 \mathrm{dBm}$ & $29.7 \mathrm{dBm}$ & $26.0 \mathrm{dBm}$ \\
Total DC power $\left(P_{d c}\right)$ & $2.1 \mathrm{~W}$ & $1.8 \mathrm{~W}$ & $1.2 \mathrm{~W}$ \\
Transducer gain $\left(G_{T}\right)$ & $10.7 \mathrm{~dB}$ & $7.2 \mathrm{~dB}$ & $7.0 \mathrm{~dB}$ \\
Power-added efficiency $(P A E)$ & $40.4 \%$ & $41.0 \%$ & $25.8 \%$ \\
Drain efficiency $(D E)$ & $44.1 \%$ & $50.6 \%$ & $32.3 \%$ \\
\hline
\end{tabular}

Table 5.3: Measured results at $P_{A V S}=19 \mathrm{dBm}$ are compared to simulated predictions at $P_{A V S}=19 \mathrm{dBm}$ and $P_{A V S}=22.5 \mathrm{dBm}$.

It is common practice to demonstrate peak quantities when evaluating power amplifier performance. That is, all published works on the topic quote gain and output power at the input power level that gives maximum power-added efficiency. Since a more conservative approach is taken in this work, maximum $P A E$ is not measured. Table 5.4 compares this work with other reported GaN-based power amplifiers of similar gate width and frequencies. GaN HEMTs operating as switching elements in Class E PAs is relatively new to the field, so most of the reported literature centers around demonstrating device capabilities in Class $\mathrm{AB}$ configurations. In the Class $\mathrm{E}$ configuration, this $\mathrm{PA}$ demonstrates comparable output power and gain to that reported by [42], but has an over $13 \%$ higher 


\begin{tabular}{|c|c|c|c|c|c|c|}
\hline PA Class & $f[\mathrm{GHz}]$ & $W_{g}[\mathrm{~mm}]$ & $P_{\text {out }}[\mathrm{dBm}]$ & $G_{T}[\mathrm{~dB}]$ & $P A E[\%]$ & Ref. \\
\hline \hline AB & 0.9 & 2 & 33 & 14 & 34 & {$[72]$} \\
\hline AB & 2 & 1 & 35 & 16 & 29 & {$[80]$} \\
\hline AB & 3.6 & $?$ & 31 & 12 & 12 & {$[81]$} \\
\hline AB & 5.5 & 1 & 27.5 & 8.5 & 22 & {$[82]$} \\
\hline E & 1.9 & 1 & 30 & 11 & 27 & {$[42]$} \\
\hline E & 3.5 & 2 & 29.74 & 10.74 & 40.4 & This work \\
\hline
\end{tabular}

Table 5.4: PA performance comparison with other reported work. All parameters are measured at a $19 \mathrm{dBm}$ input power level in order to present a fair comparison with this work. Note that $W_{g}$ is the gate width.

PAE and uses a device whose gate width is two times larger.

\subsection{Summary}

The Class E PA, first proposed by the Sokals, has endured much interest in the research community. The original circuit is fairly simple, consisting of an active device switch and a series resonant circuit for waveform shaping. The switched-mode characteristics that make this PA nonlinear also allow $100 \%$ DC-to-RF conversion at high output power levels. The Class E PA has seen applications in low power, low frequency applications, but has not been feasible in high power applications primarily due to inadequate device technology. GaN-based microwave power devices are an emerging technology well-suited for study in the Class E configuration.

The work presented in this chapter takes the familiar lumped element configuration and "transforms" it into a planar Class E PA operating in the WiMAX band at 3.5 GHz. The circuit, complete with on-board high-power bias insertion circuitry, generates an output power of $29.7 \mathrm{dBm}$ at $40.4 \%$ power-added efficiency with $10.7 \mathrm{~dB}$ of gain. A 2 mm GaN-on-Si HEMT, die-attached to a an aluminum heat sink, is used as the switching 
element. Microstrip input and output matching circuits and lumped element bias insertion units are fabricated/assembled on Rogers RT/duroid@ 5880 high-frequency substrate. The two-stage Class E load network functions to provide both a high impedance second harmonic termination and the correct Class E impedance to the drain of the HEMT. The bias insertion circuits decouple the power supply and provide a stable operating point to the PA without loading the matching circuitry. The input of the HEMT is power matched to $50 \Omega$ for $25 \mathrm{dBm}$ of input power and has a measured small-signal return loss of $6.1 \mathrm{~dB}$.

Overall, the PA performs better than predicted in simulations. Measurements indicate that the power required to switch the device between the on and off states is $3.5 \mathrm{~dB}$ lower than the original estimate, giving gain, efficiency, and output power levels comparable to simulations, but at a uniformly lower measured input power level. 


\section{Chapter 6}

\section{Conclusions and Future Work}

This research has shown the successful demonstration of GaN-on-Si transistor technology for switched-mode microwave power amplifiers. Specific conclusions are as follows.

- GaN-on-Si HEMT technology promises to be lower cost than the alternative GaNon-SiC process; however, the thermal performance of $\mathrm{Si}$ as a substrate is still inferior to that of SiC. Due to high 2DEG sheet charge densities and higher breakdown electric field levels, GaN devices are better suited to high-frequency, high-power, switched-mode power amplifier applications than established technologies, such as Si LDMOSFET and GaAs MESFET devices.

- A new large-signal equivalent model has been developed for a $2 \mathrm{~mm} \times 0.7 \mu \mathrm{m}$ GaN-on-Si HEMT at DC and over the $1-20 \mathrm{GHz}$ range, based on measured data. The chosen EEsof GaAs nonlinear HEMT model reproduces the GaN transistor's behavior reasonably well over a large range of bias conditions (drain voltages and currents up to $40 \mathrm{~V}$ and $1 \mathrm{~A}$, respectively), signal levels (up to $25 \mathrm{dBm}$ input power), and frequencies. 
- The model does not accurately predict forward gate and pinched-off Schottky diode behaviour, or the frequency dependence of extrinsic elements at the drain and gate.

- The design, optimization, fabrication and testing of a $3.5 \mathrm{GHz}, 942 \mathrm{~mW}$, Class E power amplifier have been carried out. The PA has been designed using Agilent's ADS microwave circuit simulator with a nonlinear transistor model (developed as part of this work), and has been successfully demonstrated in hybrid microstrip technology. It displayed a measured gain of $10.7 \mathrm{~dB}$, power-added efficiency of $40.4 \%$, and output power of $942 \mathrm{~mW}$. This high PAE was in good agreement with predictions, although at operating power and gain levels that were offset from the simulated values. The limitations of the model (both electrically and thermally) and the newness of the devices employed (process variations) are responsible for the differences observed.

- High power bias insertion circuits have been designed to decouple the power supply, to $\mathrm{AC}$ couple the input and output signals, and to offer a stable operating point to the PA without loading the matching circuitry.

- This work shows the suitability of low-cost GaN-on-Si transistor technology for switched-mode microwave power amplifiers with possible applications in WiMAX base stations and other communication systems.

While this thesis has been successful in achieving the objectives initially set out, it is imperative that future work focus on the following.

- Custom device modeling of AlGaN/GaN transistors is important due to deficiencies 
of currently available HEMT models that are based on other material systems such as $\mathrm{AlGaAs} / \mathrm{GaAs}$.

- Investigation of other switched-mode topologies such as Class F, Class M and Class S.

- Use of GaN devices in other parts of the communications system (low-noise receivers, phase shifter, etc.). 


\section{Appendix A}

\section{Agilent EEHEMT $2 \mathrm{~mm}$ GaN-on-Si Nonlinear Model}

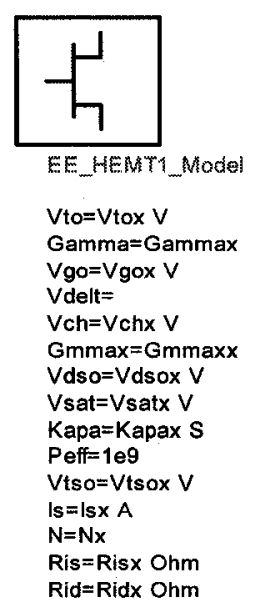

Eger VAP

$\begin{array}{ll}\text { Vtox }=-3 & \text { Cbs } x=1.6 e-13 \\ \text { Gammax }=0.0025 & \text { Vtoac }=-3.2 \\ \text { Vgox }=-1.6 & \text { Gammaacx }=0.0025 \\ \text { Veh } x=2 & \text { Gmmaxacx }=0.32 \\ \text { Gmmax }=0.33 & \text { Kapaac }=0.00001 \\ \text { Vdsox }=20 & \text { Vtsoac } x=-19 \\ \text { Vsatx }=8.5 & \text { Gdbm }=0 \\ \text { Kapax }=0.00001 & \text { Kdbx }=0 \\ \text { Vtsox }=-19 & \text { Vdsm }=1 \\ \text { Is } x=3 e-12 & \text { C110 }=3.39872 \\ \text { N } x=2.05 & \text { C11th } x=1.35871 \\ \text { Rid } x=8.22 & \text { Vinfl } x=-2.70755 \\ \text { Ris } x=2.08 & \text { Deltgs } x=0.931521 \\ \text { Taux }=7.2694 & \text { Deltds } x=6.97192 \\ \text { Cdsox }=0.28 & \text { Lambdax }=0.00267041 \\ & \end{array}$

Tau=Taux psec
Cdso=Cdsox $\mathrm{pF}$
Rdb=1e9 Ohm
Cbs=Cbsx fF
Vtoac=Vtoacx $\mathrm{V}$
Gammaac=Gammaacx
Vdeltac=
Gmmaxac=Gmmaxacx
Kapaac=Kapaacx $\mathrm{mS}$
Peffac=1e9
Vtsoac=Vtsoacx $\mathrm{V}$
Gdbm=Gdbmx
Kdb=Kdbx
Vdsm=Vdsmx
$C 110=C 110 x p F$

C11 th $=$ C11 th $x p F$ Vinfl=Vinflx $V$ Deltgs $=$ Deltgs $x V$ Deltds $=$ Deltds $x V$ Lambda $=$ Lambdax C12sat $=$ C12satx $\mathrm{pF}$ Cgdsat=Cgdsatx pF $\mathrm{Kbk}=$ $\mathrm{Vbr}=160 \mathrm{~V}$

$\mathrm{Nbr}=$

Idsoc $=$ Idsoc $A$ $\mathbf{R d}=\mathbf{R d x}$ Rs $=R s x$ $\mathrm{Rg}=\mathrm{Rg} x$

Ugw=

Ngf $=$
$V c o=V \operatorname{cox} V$
Vba=Vbax $V$
Vbc=Vbcx $V$
Mu=Mux
Deltgm=Deltgmx
Deltgmac=Deltgmac $x$
Alpha=Alphax $V$
Tnom $=$
Rgtc $=$
Rdtc $=$
Rstc=
Vtotc=
Gmmaxtc=
Gammatc=

Vinfltc $=$

Vtoactc $=$

Gmmaxactc $=$ Gammaactc $=$

$\mathrm{Xti}=$

$w V g f w d=$

wBvgs=

$\mathrm{wBvgd}=$

wBvds $=$

widsmax $=$

wPmax $=$

AllParams=
Var VAR

C12sat $x=0.283738$
Cgdsat $x=0.343952$
Idsoc $x=1.5$
$R d x=1.1$
$R s x=0$
$R g x=1.176$
Vcox $=-1.0$
Vbax $=1$
Vbc $x=5$
Mux $=0$
Deltgm $x=0.03$
Deltgmac $x=0.03$
Alphax $=8.22149 e-07$

$\operatorname{Ld} x=96.5$

$\operatorname{Lg} x=67.3$

$\operatorname{Ls} x=1.125$

Figure A.1: Agilent EEsoff HEMT nonlinear model component used in circuit simulations. 


\section{References}

[1] S.J. Vaughan-Nichols. (2005, June) Achieving wireless broadband with wimax. [Online]. Available: http://ieeexplore.ieee.org/iel5/2/28995/01306375.pdf?arnumber=1306375

[2] J. Trulove. (2005, April) Wimax's promise for developing countries. [Online]. Available: http://www.wimax.com/commentary/spotlight/wimaxspotlight2005_04_14

[3] "IEEE 802.16a standard and wimax: Igniting broadband wireless access," White Paper, Worldwide Interoperability for Microwave Access Forum. [Online]. Available: www.wimaxforum.org/news/downloads/WiMAXWhitepaper.pdf

[4] G. Sims and I. Stephenson, Microwave Tubes and Semiconductor Devices. Glasgow, UK: Blackie and Son Limited, 1963.

[5] R. Borges. (2001, September) Gallium nitride electronic devices for high-power wireless applications. 0901Borges72.pdf. [Online]. Available: http://images.rfdesign.com/files/4/

[6] TMD Technologies Ltd. (2005, April) Product summary tubes. tubes_product_summary_evd6054_issue\%202.pdf. [Online]. Available: http://www.tmd.co.uk/microwave/

[7] S. Patel. (2003, January/February) The bottleneck on the road to 3g. WirelessFuturesRoadTo3GJanFeb03.pdf. [Online]. Available: http://www.nitronex.com/media/

[8] F. Schwierz and J. Liou, Modern Microwave Transistors: Theory, Design, and Performance. Hoboken, NJ: John Wiley \& Sons, Inc., 2003.

[9] N.V. Drozdovski and R.H. Caverly, "GaN-Based High electron Mobility Transistors for Microwave and RF Control Applications," IEEE Transactions on Microwave Theory and Techniques, vol. 50, no. 1, pp. 1-8, January 2002.

[10] J. Lee, L. Eastman, and K.J. Webb, "A Gallium-Nitride Push-Pull Microwave Power Amplifier," IEEE Transactions on Microwave Theory and Techniques, vol. 51, no. 11, pp. 89-92, November 2003. 
[11] D. Kimball, P. Draxler, J. Jeong, C. Hsia, S. Lanfranco, W. Nagy, K. Linthicum, L. Larson, and P.Asbeck, "50\% PAE WCDMA basestation amplifier implemented with GaN HFETs," in Compound Semiconductor Integrated Circuits Symposium Digest, 2005, November.

[12] J.D. Brown, R. Borges, E. Piner, A Vescan, S. Singhal, and R. Therrien, "AlGaN/GaN HFETs fabricated on 100-mm GaN on silicon (111) substrates," SolidState Electronics, vol. 46, pp. 1535-1539, February 2002.

[13] N.O. Sokal and A.D. Sokal, "High-Efficiency Tuned Switching Power Amplifier," U.S. Patent 3919656, Nov. 11, 1975.

[14] P.M. Smith, S.-M.J. Liu, M.-Y. Kao, P. Ho, S.C. Wang, K.H.G. Duh, S.T. Fu, and P.C. Chao, "W-Band High Efficiency InP-Based Power HEMT with $600 \mathrm{GHz} f_{m} a x$," IEEE Microwave and Guided Wave Letters, vol. 5, no. 7, 1995.

[15] K. Inoue, K. Ebihara, H. Haematsu, T. Igarashi, H. Takahashi, and J. Fukaya, "A 240 W Push-Pull Power FET for W-CDMA Base Stations," IEEE Microwave Symposium Digest, vol. 3, pp. 1719-1722, June 2000.

[16] A.N. Lepore, H.M. Levy, R.C. Tiberio, P.J. Tasker, H. Lee. E.D. Wolf, L.F. Eastman, and E. Khon, "0.1 $\mu \mathrm{m}$ Gate Length MODFETs with Unity Current Gain Cutoff Frequency Above 110 GHz," IEEE Electronics Letters, vol. 24, pp. 364-366, March 1988.

[17] S.E. Rosenbaum, B.K. Kormanyos, L.M. Jelloian, M. Matloubian, A.S. Brown, L.E. Larson, L.D. Nguyen, M.A. Thompson, L.P.B. Katehi, and G.M. Rebeiz, "155- and 213-GHz AlInAs/GaInAs/InP HEMT MMIC Oscillators," IEEE Transactions on Microwave Theory and Techniques, vol. 47, pp. 927-932, April 1995.

[18] T. Palacios, A. Chakraborty, S. Heikman, S. Keller, S.P. DenBaars, and U.K. Mishra, "AlGaN/GaN High Electron Mobility Transistors With InGaN Back-Barriers," IEEE Electron Device Letters, vol. 27, no. 1, pp. 13-15, January 2006.

[19] P. Javorka, A. Alam, A. Fox, M. Marso, M. Heuken, and P. Kordos, "AlGaN/GaN HEMTs on Silicon Substrates with $f_{T}$ of $32 / 20 \mathrm{GHz}$ and $f_{\max }$ of $27 / 22 \mathrm{GHz}$ for 0.5/0.7 $\mu \mathrm{m}$ Gate Length," IEEE Electronics Letters, vol. 38, no. 6, pp. 288-289, March 2002.

[20] Y. Tateno, H. Takahashi, T. Igarashi, and J. Fukaya, "A 150 W E-Mode GaAs Power FET with 35\% PAE for W-CDMA Base Station," IEEE Microwave Symposium Digest, vol. 3, pp. 1087-1090, June 1999.

[21] R. Grundbacher, M. Nishimoto, T.P. Chin, Y.C. Chen, R. Lai, and D. Yamauchi, "InP Power HEMTs With 36\% PAE at $60 \mathrm{GHz}$," Eleventh International Conference on Indium Phosphide and Related Materials, pp. 307-310, May 1999. 
[22] Y.-F. Wu, A. Saxler, M. Moore, R.P. Smith, S. Sheppard, P.M. Chavarkar, T. Wisleder, U.K. Mishra, and P. Parikh, "30-W/mm GaN HEMTs by Field Plate Optimization," IEEE Electron Device Letters, vol. 25, no. 3, pp. 117-119, March 2004.

[23] J.W. Johnson, E.L. Piner. A. Vescan, R. Therrien, P. Rajagopal, J.C. Roberts, J.D. Brown, S. Singhal, and K.J. Linthicum, "12 W/mm AlGaN-GaN HFETs on Silicon Substrates," IEEE Electron Device Letters, vol. 25, no. 7, pp. 459-461, July 2004.

[24] M. Matloubian, L.D. Nguyen, A.S. Brown, L.E. Larson, M.A. Melendes, and M.A. Thompson, "High Power and High Efficiency AlInAs/GaInAs on InP HEMTs," IEEE Microwave Symposium Digest, vol. 2, pp. 721-724, June 1991.

[25] C. Nguyen and M. Micovic, "The State-of-the-Art of GaAs and InP Power Devices and Amplifiers," IEEE Transactions on Electron Devices, vol. 48, no. 3, pp. 472-478, March 2001.

[26] S. Cripps, RF Power Amplifiers for Wireless Communications. Norwood, MA: Artech House, Inc., 1999.

[27] J. Rogers and C. Plett, Radio Frequency Integrated Circuit Design. Norwood, MA: Artech House, Inc., 2003.

[28] H.L. Krauss, C.W. Bostian, and F.H. Raab, Solid State Radio Engineering. New York, NY: John Wiley \& Sons, Ltd., 1980.

[29] T. Lee, The Design of CMOS Radio-Frequency Integrated Circuits, 2nd ed. New York, NY: Cambridge University Press, 2004.

[30] S. McGarry, private communication, 2006.

[31] N.O. Sokal and A.D. Sokal, "Class E - A New Class of High-Efficiency Tuned SingleEnded Switching Power Amplifiers," IEEE Journal of Solid-State Circuits, vol. SC10, no. 3, pp. 168-176, June 1975.

[32] S.C. Cripps, RF Power Amplifiers for Wireless Communications, 2nd ed. Norwood, MA: Artech House, Inc., 2006.

[33] N. Sokal, "Class-E high-efficiency power amplifiers, from HF to microwave," in Proceedings of the IEEE International Microwave Symposium, Boston, MA, June 2000, pp. 1109-1112.

[34] F.H. Raab, "Idealized Operation of the Class E Tuned Power Amplifier," IEEE Transactions on Circuits and Systems, vol. CAS-24, no. 12, 1977.

[35] M.K. Kazimierczuk and K. Puczko, "Exact Analysis of Class E Tuned Power Amplifier at any Q and Switch Duty Cycle," IEEE Transactions on Circuits and Systems, vol. CAS-34, no. 2, 1987. 
[36] D.K. Choi, "High Efficiency Switched-Mode Power Amplifiers for Wireless Communications," Ph.D. dissertation, University of California, Santa Barbara, March 2001.

[37] F.J. Ortega-Gonzalez, "New Driver for High-Efficiency Switching RF Power Amplifiers," IEEE Microwave and Optical Technology Letters, vol. 43, no. 5, pp. 370-372, December 2004.

[38] T.B. Mader, "Quasi-Optical Class-E Power Amplifiers," Ph.D. dissertation, University of Colorado, Boulder, August 1995.

[39] J. Rollett, "Stability and Power-Gain Invariants of Linear Twoports," IRE Transactions on Circuit Theory, vol. 9, pp. 29-32, March 1962.

[40] W. Saito, T. Domon, I. Omura, M. Karaguchi, Y. Takada, K. Tsuda, and M. Yamaguchi, "Demonstration of 13.56-MHz class-e amplifier using a high-voltage GaN power-HEMT," IEEE Electron device Letters, vol. 27, no. 5, pp. 326-328, May 2006.

[41] W. Saito, M. Karaguchi, Y. Takada, K. Tsuda, T. Domon, I. Omura, and M. Yamaguchi, "Two-stage quasi-class-e power amplifier in GaN HEMT technology," IEEE Microwave and Component Letters, vol. 16, no. 1, pp. 28-30, January 2006.

[42] H. Xu, S. Gao, S. Heikman, S.I. Long, U.K. Mishra, and R.A. York, "A high-efficiency class-e GaN HEMT power amplifier at $1.9 \mathrm{GHz}$," IEEE Microwave and Component Letters, vol. 16, no. 1, pp. 22-24, January 2006.

[43] S. Gao, H. Xu, S. Heikman, U.K. Mishra, R.A. York, "Two-stage quasi-class-e power amplifier in GaN HEMT technology," IEEE Microwave and Component Letters, vol. 16 , no. 1 , pp. 28-30, January 2006.

[44] R. P. W.L. Pribble, J.M. Milligan. (2006) High efficiency class-e amplifier utilizing gan hemt technology. Cree Inc. Durham, NC. [Online]. Available: www.cree.com

[45] W. Curtice, "A MESFET model for use in the design of GaAs integrated circuits," IEEE Transactions on Microwave Theory and Techniques, vol. MTT-28, no. 5, pp. 448-455, May 1980.

[46] K. Koh, H. Park, and S. Hong, "A spline large signal FET model based on bias dependent pulsed I-V measurements," IEEE Transactions on Microwave Theory and Techniques, vol. 28, no. 5, pp. 448-455, May 2002.

[47] M. Reiser, "A Two-Dimensional Numerical FET Model for DC, AC, and LargeSignal Analysis," IEEE Transactions on Electron Devices, vol. ED-20, no. 1, pp. 35-45, January 1973.

[48] J.J. Barnes, R.J. Lomax, G.I. Haddad, "Finite-Element Simulation of GaAs MESFETs with Lateral Doping Profiles and Submicron Gates," IEEE Transactions on Electron Devices, vol. ED-23, no. 9, pp. 1042-1048, September 1976. 
[49] A. Grebennikov, $R F$ and Microwave Power Amplifier Design. U.S.A.: The McGrawHill Companies, Inc., 2005.

[50] J. Golio, Microwave MESFETs 8 HEMTs. Norwood, MA: Artech House, Inc., 1991.

[51] A. Materka and T. Kacprzak, "Computer Calculation of Large-Signal GaAs FET Amplifier Characteristics," IEEE Transactions on Microwave Theory and Techniques, vol. MTT-33, no. 2, pp. 129-135, February 1985.

[52] W. Curtice and M. Ettenberg, "A nonlinear GaAs FET model for use in the design of output circuits for power amplifiers," IEEE Transactions on Microwave Theory and Techniques, vol. MTT-33, no. 12, pp. 1383-1393, December 1985.

[53] H. Statz, P. Newman, I. Smith, R. Pucel, and H. Haus, "GaAs FET device and circuit simulation in spice," IEEE Transactions on Electron Devices, vol. ED-34, pp. 160-169, February 1987.

[54] Agilent Technologies Inc., Nonlinear Devices. Palo Alto, CA: Agilent Technologies Inc., 2005, ch. 3.

[55] "Nitronex:Core Competencies:Crystal Growth," coreComp.html, Nitronex Corporation, 2007. [Online]. Available: http://www.nitronex.com/about/

[56] P. Canfield, S. Lam, and D. Allstot, "Modeling of frequency and temperature effects in GaAs MESFETs," IEEE Journal of Solid-State Circuits, vol. 25, no. 1, pp. 299305, February 1990.

[57] J. Golio, M. Miller, G. Maracas, and D. Johnson, "Frequency-dependent electrical characteristics of GaAs MESFETs," IEEE Transactions on Electron Devices, vol. 37, no. 5, pp. 1217-1226, May 1990.

[58] P.Ladbrooke and J. Bridge, "The Importance of the Current-Voltage Characteristics of FETs, HEMTs, and Bipolar Transistors in Contemporary Circuit Design," Microwave Journal, March 2002.

[59] S. Manohar, A. Narayanan, A.Keerti, A. Pham, J. Brown, R. Borges, and K. Linthicum, "Characteristics of Microwave Power GaN HEMTs on 4-Inch Si Wafers," IEEE Microwave Symposium Digest, vol. 1, pp. 449-452, June 2002.

[60] R.G. Trew, G.L. Bilbro, W. Kuang, Y. Liu, and H. Yin, "Microwave AlGaN/GaN HFETs," IEEE Microwave Magazine, vol. 6, no. 1, pp. 56-66, March 2005.

[61] S. De Meyer, C. Charbonniaud, R. Quéré, M. Campovecchio, R. Lossy, and J. Würfl, "Mechanism of Power Density Degradation due to Trapping Effects in AlGaN/GaN HEMTs," IEEE Microwave Symposium Digest, vol. 1, pp. 455-458, June 2003. 
[62] S.C. Binari, K. Ikossi, J.A. Roussos, W. Kruppa, D. Park, H.B. Dietrich, D.D. Koleske, A.E. Wickenden, and R.L. Henry, "Trapping Effects and Microwave Performance in AlGaN/GaN HEMTs," IEEE Transactions on Electron Devices, vol. 48, no. $3,2001$.

[63] T. Mizutani, Y. Ohno, M. Akita, S. Kishimoto, and K. Maezawa, "A Study on Current Collapse in AlGaN/GaN HEMTs Induced by Bias Stress," IEEE Transactions on Electron Devices, vol. 50, no. 10, 2003.

[64] A.F.M. Anwar, S. Wu, and T. Webster, "Temperature Dependent Transport Properties in $\mathrm{GaN}, \mathrm{Al}_{\mathrm{x}} \mathrm{Ga}_{1-\mathrm{x}} \mathrm{N}$, and $\mathrm{In}_{\mathrm{x}} \mathrm{Ga}_{1-\mathrm{x}} \mathrm{N}$ Semiconductors," IEEE Transactions on Electron Devices, vol. 48, no. 3, 2001.

[65] C. Wei, Y.A. Tkachenko, and D. Bartle, "An Accurate Large-Signal Model of GaAs MESFET Which Accounts for Charge Conservation, Dispersion, and Self-Heating," IEEE Transactions on Microwave Theory and Techniques, vol. 46, no. 11, pp. 16381644, November 1998.

[66] M.J. Uren, T. Martin, J.C. Birbeck, R. Balmer, B.T. Highes, A.J. Hydes, H. Willis, T. Jelfs, "GaN/AlGaN HFETs Fabricated on a SiC Substrate," in Eighth IEEE International Symposium on High Perfomance Electron Devices for Microwave and Optoelectronic Applications, Glasgow, United Kingdom, November.

[67] A. Sedra and K. Smith, Microelectronic Cicruits, 4th ed. New York, NY: Oxford University Press, Inc., 1998.

[68] A. Chaudhari, private communication, 2005.

[69] G. Dambrine, A. Cappy, F. Heliodore, and E. Playez, "A new method for determining the FET small-signal equivalent circuit," IEEE Transactions on Microwave Theory and Techniques, vol. 36, no. 7, pp. 1151-1159, July 1988.

[70] Agilent Technologies. (2001, July) High Speed DC Characterization of Semiconductor Devices from Sub pA to 1A. 59502930.pdf. [Online]. Available: http://www.home.agilent.com/upload/cmc_upload/All/

[71] J.M. Shah, Y. Li, T. Gessmann, and E.F. Schubert, "Experimental analysis and theoretical model for anomalously high ideality factors $(n \gg 2.0)$ in AlGaN/GaN p-n junction diodes," Journal of Applied Physics, vol. 94, no. 4, 2003.

[72] P.M. Cabral, J.C. Pedro, and N.B. Carvalho, "Nonlinear Device Model of Microwave Power GaN HEMTs for High Power-Amplifier Design," IEEE Transactions on Microwave Theory and Techniques, vol. 52, no. 11, pp. 2585-2592, November 2004.

[73] T.B. Mader and Z. Popović, "The Transmission-Line High-Efficiency Class-E Amplifier," IEEE Microwave and Guided Wave Letters, vol. 5, no. 9, pp. 290-292, September 1995. 
[74] T.B. Mader, E.W. Bryerton, M. Marković, M. Forman, and Z. Popović, "SwitchedMode High Efficiency Microwave Power Amplifiers in a Free-Space Power-Combiner Array," IEEE Transactions on Microwave Theory and Techniques, vol. 46, no. 10, pp. 1391-1397, October 1998.

[75] E.W. Bryerton, M.D. Weiss, and Z. Popović, "Efficiency of Chip-Level Versus External Power Combining," IEEE Transactions on Microwave Theory and Techniques, vol. 47, no. 8, pp. 1482-1485, October 1999.

[76] M.D. Weiss, M.H. Crites, E.W. Bryerton, J.F. Whitaker, and Z. Popović, "TimeDomain Optical Sampling of Switched-Mode Microwave Amplifiers and Multipliers," IEEE Transactions on Microwave Theory and Techniques, vol. 47, no. 12, pp. 25992604, December 1999.

[77] M.K. Kazimierczuk, "Generalization of Conditions for 100-Percent Efficiency and Nonzero OutputPower in Power Amplifiers and Frequency Multipliers," IEEE Transactions on Circuits and Systems, vol. CAS-33, no. 8, 1986.

[78] G. Gonzalez, Microwave Transistor Amplifiers: Analysis and Design, 2nd ed. Upper Saddle River, NJ: Prentice-Hall, Inc., 1997.

[79] R. Fiore. (2005, January) Capacitors in Broadband Applications. caps_broadbnd_apps.pdf. [Online]. Available: http://www.atceramics.com/technicalnotes/technotes.asp

[80] Y. Ando, Y Okamoto, K. Hataya, T. Nakayama, H. Miyamoto, T. Inoue, and M. Kuzuhara, "12 W/mm Recessed-Gate AlGaN/GaN Heterojunction Field-Plate FET," Electron Devices Meeting, IEDM '03 Technical Digest, pp. 23.1.1-23.1.4, December 2003.

[81] S. Wood, P. Smith, W. Pribble, R. Pengelly, and J. Crescenzi. (2006, May) High Efficiency, High Linearity GaN HEMT Amplifiers for WiMAX Applications. HFE0506_Cree.pdf. [Online]. Available: http://www.highfrequencyelectronics.com/Archives/May06/

[82] P. Colantonio, F. Giannini, R. Giofrè, E. Limiti, A. Serino, M. Peroni, P. Romanini, and C. Proietti, "A C-band high-efficiency second-harmonic-tuned hybrid power amplifier in GaN technology," IEEE Transactions on Microwave Theory and Techniques, vol. 54, no. 6, pp. 2713-2722, June 2006. 